\title{
Peridynamics for the thermomechanical behavior of shell structures
}

\section{Cong Tien Nguyen ${ }^{a, b}$ and Selda Oterkus ${ }^{a, *}$}

${ }^{a}$ Department of Naval Architecture, Ocean and Marine Engineering, University of Strathclyde, Glasgow, G11XQ, UK

${ }^{\mathrm{b}}$ Faculty of Shipbuilding, Vietnam Maritime University, Haiphong, Vietnam

\begin{abstract}
A novel peridynamic model for predicting thermomechanical behaviour of three-dimensional shell structures with 6 degrees of freedom has been proposed. Also, a numerical algorithm for dealing with complex shell structures is provided for the first time in the peridynamic literature. The peridynamic damage criterion based on critical energy release rate is provided for shell structures. The capability of the developed peridynamic model is demonstrated by predicting deformations for a flat shell, a curved shell, and a stiffened structure. To further demonstrate the capabilities of the proposed model, damage on flat shell in a double torsion problem, flat shell with pre-existing crack, thermal fracturing of a glass cup and damage in a dropped egg are simulated.
\end{abstract}

Keywords: peridynamics; thermomechanical; shell structures; damage; nonlocal 


\section{Nomenclature}

\section{Latin Letters}

$a_{i p 1}, a_{i p 2}, a_{i p 3}, b_{i p}, d_{i p}$ $a_{b}, b_{b}, d_{b}$

$\mathbf{b}, \overline{\mathbf{b}}$

$C_{s}$

$\mathrm{C}_{(k)}$

E

$\mathbf{f}_{(k)(j)}$

$\mathbf{f}_{(k)(j)}^{\prime}$

F

$G_{c}$

$g_{c}$

$\bar{g}_{(k)(j)}$

$h$

$\mathbf{H}_{(k)}, \mathbf{H}_{(k)(j)}$

$k_{s}, k_{T}$

$L, U, T$

$\overline{\mathbf{m}}_{(k)}^{\prime}$

$\overline{\mathbf{m}}_{(k)}$

$\mathbf{M}_{(k)}$

$\vec{n}_{x^{\prime}}, \vec{n}_{y^{\prime}}, \vec{n}_{z^{\prime}}$

$N_{c}$

$S_{i p(k)(j)}$

$S_{b(k)(j)}$

$\mathbf{t}, \mathbf{t}^{\prime}$

$\mathbf{T}_{(k)}, \mathbf{T}_{(k)(j)}$

$u, v, w$

$u^{\prime}, v^{\prime}, w^{\prime}$

$W$

$\bar{W}$

$\bar{W}_{\text {inplane }}$

$\bar{W}_{\text {bending }}$

$\bar{W}_{\text {shear }}$

$\bar{W}_{\text {torsional }}$
Peridynamic constants for in-plane deformations

Peridynamic constants for bending deformations

Body force, force per unit area

Peridynamic constant for the shear deformations

Damping matrix

Young's modulus

Vector of peridynamic forces in global coordinate system

Vector of peridynamic forces in local coordinate system

Deformation gradient

Critical energy release rate of material

Average critical energy release rate for one interaction

Energy release rate for interaction between material points $k$ and $j$ in peridynamics

Shell's thickness

Coordinate transformation matrices for flat shell, curved shell

Shear correction factor, torsional constant

Lagrangian, kinetic energy, total potential energy

Mass matrix in local coordinates

Mass matrix in global coordinates

Mass stable vector

Unit (director) vectors for a material point

Total number of interactions passing through a unit crack area

Relative bond stretch of in-plane deformations

Relative bond stretch of bending deformations

Peridynamic force densities

Displacement transformation matrices

Global displacements in $x, y$ and $z$ directions, respectively

Local displacements in $x^{\prime}, y^{\prime}$ and $z^{\prime}$ directions, respectively

Strain energy density

Strain energy per unit area

Strain energy per unit area for in-plane deformations

Strain energy per unit area for bending deformations

Strain energy per unit area for shear deformations

Fictitious strain energy per unit area for torsional deformations 
$x, y, z$

$x^{\prime}, y^{\prime}, z^{\prime}$

\section{Greek Letters}

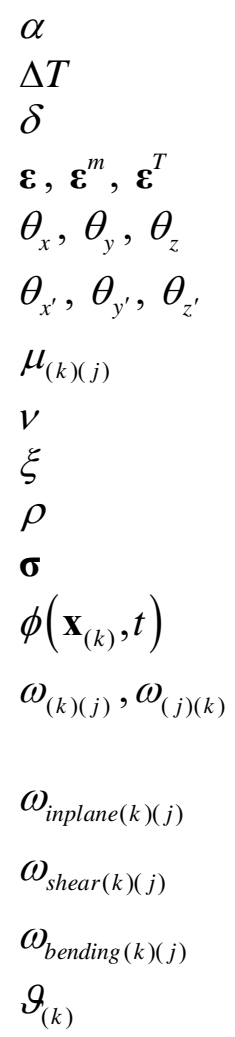

\section{Acronyms}

$\mathrm{CCM}$

DOF

FEA

PD

SED
Global coordinates

Local coordinates
Linear thermal expansion coefficient

Temperature change

Horizon size

total, mechanical, thermal strain vectors

Global rotations around $x, y$ and $z$ axis, respectively

Local rotations around $x^{\prime}, y^{\prime}$ and $z^{\prime}$ axis, respectively

Function to represent state of interaction (damaged or intact)

Poisson's ratio

Bond length in initial (undeformed) configuration

Mass density

Cauchy stress

Local damage coefficient

Micropotentials of the interaction between material point $k$ and $j$

Micropotentials for in-plane deformations

Micropotentials for shear deformations

Micropotentials for bending deformations

Dilatation of material point $k$

Classical Continuum Mechanics

Degree(s) of Freedom

Finite Element Analysis

Peridynamics

Strain Energy Density 


\section{Introduction}

Predicting damage processes such as crack initiation and propagation is a challenging work that requires much effort. The classical continuum mechanics (CCM) faces conceptual and mathematical difficulties in terms of predicting crack nucleation and growth especially for multiple crack paths since partial-differential equations become invalid in presence of discontinuities. The peridynamic (PD) formulation of continuum mechanics represents the material behavior by using integro-differential equations, which are valid in both continuous and discontinuous models [1]. Therefore, discontinuities can be naturally involved in the PD analysis without any special treatment.

The original PD theory was introduced by Silling [1] and Silling and Askari [2] to predict the mechanical behavior of three-dimensional solid objects. However, it is computationally expensive to implement 3D PD models for plates, shells and slender structures. Therefore, structural idealization is needed to reduce the computational cost. The structural idealization for plates and shell structures are well understood in classical continuum mechanics [3-6]. The behaviour of these structures are well represented by using numerical techniques such as finite element analyses [712]. In peridynamics, the first simplified PD model for two-dimensional configurations introduced by Silling and Bobaru [13], and later by Madenci and Oterkus [14], Breitenfeld et al. [15], Sarego et al. [16]. Their PD models include two degrees of freedom (DOF). In order to include transverse displacement caused by bending, O'Grady and Foster [17] introduced a non-ordinary state-based PD model which is based on Kirchhoff-Love plate theory by disregarding the transverse shear deformations. PD model developed by the authors can capture the behavior of thin, flat plates with three DOFs which are three displacement components. In order to include transverse shear deformations, later, Diyaroglu et al. [18] developed a bond based PD model for plate based on Mindlin plate theory. The developed PD model can capture the behavior of a plate with three DOFs including transverse displacement and two rotations. They also developed a bond based PD model for plane beams with two degrees of freedom to predict bending deformations. Recently, Nguyen and Oterkus [19] developed a PD model for 3D beam structures with six degrees of freedom to predict the damages in offshore structures. The bond based PD model for beam and plate developed by Diyaroglu et al. [18] is implemented in finite element framework by Yang et al. [20]. Chowdhury et al. [21] developed a state based peridynamic model for linear elastic shells by introducing curved bond definition.

The effect of temperature on mechanical deformations is also considered in peridynamic framework. Kilic and Madenci [22] introduced bond based PD model for one-way coupled thermomechanics. Later, bond based PD models for fully coupled thermo-mechanics were introduced in [23-25]. Amani et al. [26] introduced non-ordinary state-based PD model for thermoplastic problems and Madenci and Oterkus [27, 28] introduced ordinary-state based model for plastic and thermo-viscoelastic deformations. Recently, Gao and Oterkus [29,30] developed ordinary statebased PD model for fully coupled thermo-elastic problems. The developed PD models are used to predict damages due to thermal effects [31, 32]. Xu et al. [33] studied elastic vortices and thermally-driven cracks in brittle materials by using peridynamics. However, in these PD models the effect of bending deformations are not included. Therefore, this study focuses on developing a novel ordinary state based peridynamic model to predict the thermomechanical behaviour of threedimensional shell structures with 6 degrees of freedom. The numerical technique and procedure to deal with complex shell structures are also provided. For verification purposes, static analyses of a flat shell, a curved shell, and a stiffened structure are presented. The proposed PD model is used 
to predict damages in a flat shell subjected to double torsion loading, flat shell with pre-existing crack, damages in a glass cup subjected to temperature change and damages in a dropped egg.

This paper is organized as follows. Section 2 presents the classical continuum mechanics formulations for linear strain, linear stress components and strain energy density for shell structures. Section 3 provides the PD strain energy density and PD equations of motion for shell structures. Section 4 presents the transformation of PD equations of motion from local to global coordinates for both flat and curved shells. Section 5 provides a criterion to predict damage on shell structures by using the proposed PD model. The numerical algorithm and numerical techniques to deal with connections of complex shell structures are provided in Section 6. Section 7 presents the PD results, followed by the conclusions in Section 8.

\section{Kinematics of Flat Shells in Classical Continuum Mechanics}

In this section, first, the descriptions of degrees of freedom for a material point on a shell structure are provided. Next, the formulations for linear strain and stress are obtained. Finally, the strain energy density formulation in classical continuum mechanics for shell structures is presented.

\subsection{Degrees of Freedom}

The kinematics of flat shell is initially based on the theory of plates by including transverse shear deformations [3-5]. According to assumptions in [3-5], the deformation of each material point in a plate is represented by five degrees of freedom. Later, Wisniewski, [6] added one more degree of freedom, which is the rotation with respect to normal axis of the plate's plane.

Fig. 1 presents a material point in a flat shell with 6 degrees of freedom; $u, v, w, \theta_{x}, \theta_{y}, \theta_{z}$ in the global and $u^{\prime}, v^{\prime}, w^{\prime}, \theta_{x^{\prime}}, \theta_{y^{\prime}}, \theta_{z^{\prime}}$ in the local coordinate systems with respect to undeformed configuration. The components $u^{\prime}, v^{\prime}$ represent the in-plane displacements of the mid-surface in $x^{\prime}, y^{\prime}$ directions, respectively. Meanwhile, $w^{\prime}$ represents the transverse displacement of the middle surface in $z^{\prime}$ direction. The rotations $\theta_{x^{\prime}}, \theta_{y^{\prime}}, \theta_{z^{\prime}}$ represent the rotations of the plate around $x^{\prime}, y^{\prime}$ and $z^{\prime}$, respectively. The. The drilling rotation, $\theta_{z^{\prime}}$ can be found from the rotational constraint equation as [6]

$\operatorname{skew}\left(\mathbf{Q}^{T} \mathbf{F}\right)=0$

where

$\mathbf{Q}=\left[\begin{array}{cc}\cos \theta_{z^{\prime}} & -\sin \theta_{z^{\prime}} \\ \sin \theta_{z^{\prime}} & \cos \theta_{z^{\prime}}\end{array}\right]$

$\mathbf{F}=\left[\begin{array}{cc}1+u_{, x^{\prime}}^{\prime} & u_{, y^{\prime}}^{\prime} \\ v_{, x^{\prime}}^{\prime} & 1+v_{, y^{\prime}}^{\prime}\end{array}\right]$

The rotational constraint given in Eq. (1a) can also be written as

$-\left(v_{, y^{\prime}}^{\prime}+u_{, x^{\prime}}^{\prime}+2\right) \sin \theta_{z^{\prime}}+\left(v_{, x^{\prime}}^{\prime}-u_{, y^{\prime}}^{\prime}\right) \cos \theta_{z^{\prime}}=0$

If $\theta_{z^{\prime}} \neq \pi / 2$, Eq. (2) becomes [6]

$\theta_{z^{\prime}}=\arctan \left(\frac{v_{, x^{\prime}}^{\prime}-u_{, y^{\prime}}^{\prime}}{v_{, y^{\prime}}^{\prime}+u_{, x^{\prime}}^{\prime}+2}\right)$ 
For small rotation and small strain conditions, Eq. (3) becomes $\theta_{z^{\prime}}=\frac{1}{2}\left(v_{, x^{\prime}}^{\prime}-u_{, y^{\prime}}^{\prime}\right)$

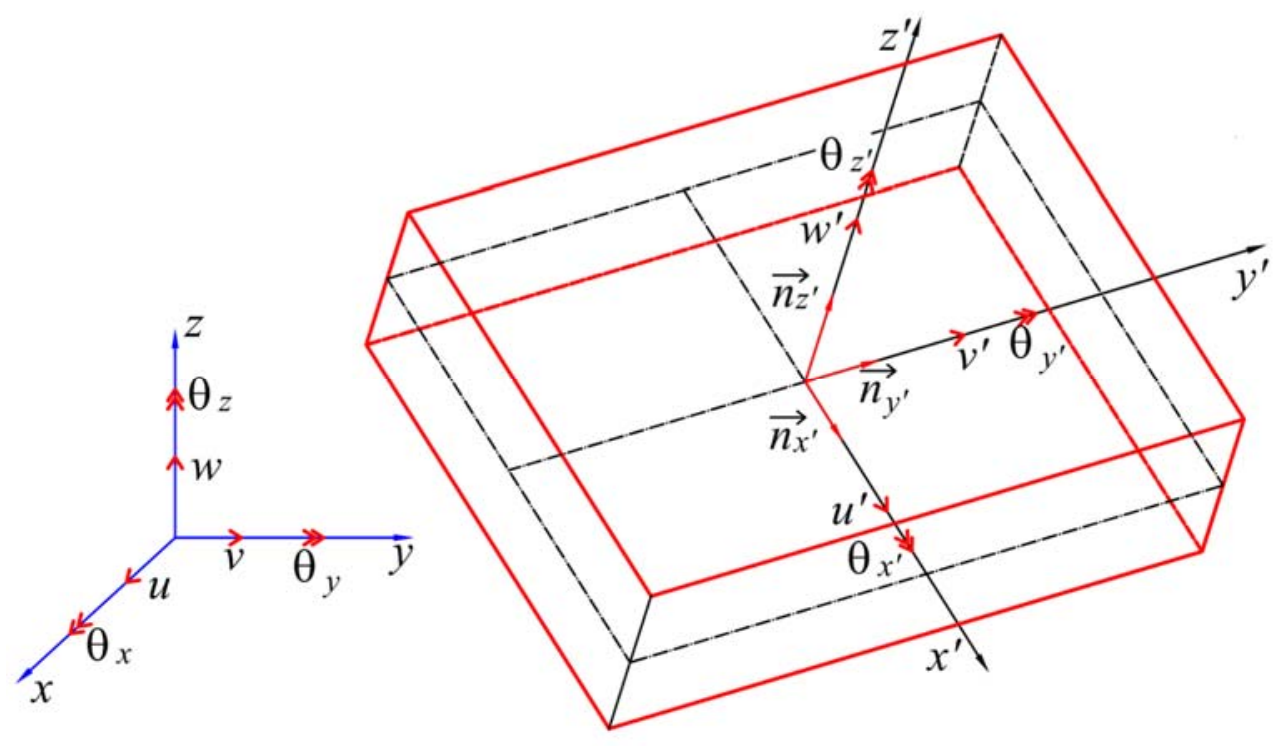

Fig. 1. Material point in a flat shell with 6 degrees of freedom

\subsection{Linear Strain Components}

For small deformations, the displacement components of a point located at $\left(x^{\prime}, y^{\prime}, z^{\prime}\right)$ with respect to mid-surface can be expressed as $[3-5,10]$

$\hat{u}^{\prime}\left(x^{\prime}, y^{\prime}, z^{\prime}\right)=u^{\prime}\left(x^{\prime}, y^{\prime}\right)+z^{\prime} \theta_{y^{\prime}}$

$\hat{v}^{\prime}\left(x^{\prime}, y^{\prime}, z^{\prime}\right)=v^{\prime}\left(x^{\prime}, y^{\prime}\right)-z^{\prime} \theta_{x^{\prime}}$

$\hat{w}^{\prime}\left(x^{\prime}, y^{\prime}, z^{\prime}\right)=w^{\prime}\left(x^{\prime}, y^{\prime}\right)$

where $u^{\prime}, v^{\prime}, w^{\prime}$ represent the displacement components of a point located at the mid-surface and $\hat{u}^{\prime}, \hat{v}^{\prime}, \hat{w}^{\prime}$ represent the displacement components at any point.

By using the displacement components given in Eq. (5), the linear strain components can be determined as

$\boldsymbol{\varepsilon}=\left[\begin{array}{lllll}\varepsilon_{x^{\prime} x^{\prime}} & \varepsilon_{y^{\prime} y^{\prime}} & \gamma_{x^{\prime} y^{\prime}} & \gamma_{x^{\prime} z^{\prime}} & \gamma_{y^{\prime} z^{\prime}}\end{array}\right]^{T}$

where

$$
\begin{aligned}
& \varepsilon_{x^{\prime} x^{\prime}}=\partial \hat{u}^{\prime} / \partial x^{\prime}=u_{, x^{\prime}}^{\prime}+z^{\prime} \theta_{y^{\prime}, x^{\prime}} \\
& \varepsilon_{y^{\prime} y^{\prime}}=\partial \hat{v}^{\prime} / \partial y^{\prime}=v_{, y^{\prime}}^{\prime}-z^{\prime} \theta_{x^{\prime}, y^{\prime}} \\
& \gamma_{x^{\prime} y^{\prime}}=\partial \hat{u}^{\prime} / \partial y^{\prime}+\partial \hat{v}^{\prime} / \partial x^{\prime}=u_{, y^{\prime}}^{\prime}+v_{, x^{\prime}}^{\prime}+z^{\prime}\left(\theta_{y^{\prime}, y^{\prime}}-\theta_{x^{\prime}, x^{\prime}}\right) \\
& \gamma_{x^{\prime} z^{\prime}}=\partial \hat{u}^{\prime} / \partial z^{\prime}+\partial \hat{w}^{\prime} / \partial x^{\prime}=w_{, x^{\prime}}^{\prime}+\theta_{y^{\prime}} \\
& \gamma_{y^{\prime} z^{\prime}}=\partial \hat{v}^{\prime} \partial \partial z^{\prime}+\partial \hat{w}^{\prime} / \partial y^{\prime}=w_{, y^{\prime}}^{\prime}-\theta_{x^{\prime}}
\end{aligned}
$$


Note that based on small deformation assumption in linear elasticity, the strain component in the thickness direction of the shell can be neglected, $\varepsilon_{z^{\prime} z^{\prime}}=0$ [10].

If the temperature effect is considered, total strain components given in Eq. (6) can be determined as a summation of mechanical strain, $\boldsymbol{\varepsilon}^{m}$ and thermal strain, $\boldsymbol{\varepsilon}^{T}$ as [34]

$\boldsymbol{\varepsilon}=\boldsymbol{\varepsilon}^{m}+\boldsymbol{\varepsilon}^{T}$

with

$\boldsymbol{\varepsilon}^{T}=\left[\begin{array}{lllll}\alpha \Delta T & \alpha \Delta T & 0 & 0 & 0\end{array}\right]^{T}$

where $\alpha$ represents the coefficient of linear thermal expansion and $\Delta T$ represents the temperature change. Therefore, the mechanical strain components can be defined as

$\boldsymbol{\varepsilon}^{m}=\boldsymbol{\varepsilon}-\boldsymbol{\varepsilon}^{T}=\left[\begin{array}{lllll}\varepsilon_{x^{\prime} x^{\prime}}^{m} & \varepsilon_{y^{\prime} y^{\prime}}^{m} & \gamma_{x^{\prime} y^{\prime}}^{m} & \gamma_{x^{\prime} z^{\prime}}^{m} & \gamma_{y^{\prime} z^{\prime}}^{m}\end{array}\right]^{T}$

where

$\varepsilon_{x^{\prime} x^{\prime}}^{m}=u_{, x^{\prime}}^{\prime}+z^{\prime} \theta_{y^{\prime}, x^{\prime}}-\alpha \Delta T$

$\varepsilon_{y^{\prime} y^{\prime}}^{m}=v_{, y^{\prime}}^{\prime}-z^{\prime} \theta_{x^{\prime}, y^{\prime}}-\alpha \Delta T$

$\gamma_{x^{\prime} y^{\prime}}^{m}=\gamma_{x^{\prime} y^{\prime}}=u_{, y^{\prime}}^{\prime}+v_{, x^{\prime}}^{\prime}+z^{\prime}\left(\theta_{y^{\prime}, y^{\prime}}-\theta_{x^{\prime}, x^{\prime}}\right)$

$\gamma_{x^{\prime} z^{\prime}}^{m}=\gamma_{x^{\prime} z^{\prime}}=w_{x^{\prime}}^{\prime}+\theta_{y^{\prime}}$

$\gamma_{y^{\prime} z^{\prime}}^{m}=\gamma_{y^{\prime} z^{\prime}}=w_{, y^{\prime}}^{\prime}-\theta_{x^{\prime}}$

\subsection{Stress Components}

For a linear elastic isotropic, homogeneous material with $\sigma_{z^{\prime}}=0$, the stress components can be expressed as [34]

$\boldsymbol{\sigma}=\mathbf{D} \boldsymbol{\varepsilon}-\mathbf{D} \boldsymbol{\alpha} \Delta T$

with

$\mathbf{D}=\frac{E}{1-v^{2}}\left[\begin{array}{ccccc}1 & v & 0 & 0 & 0 \\ v & 1 & 0 & 0 & 0 \\ 0 & 0 & \frac{1-v}{2} & 0 & 0 \\ 0 & 0 & 0 & \frac{1-v}{2} & 0 \\ 0 & 0 & 0 & 0 & \frac{1-v}{2}\end{array}\right]$

$\boldsymbol{\alpha}=\left[\begin{array}{lllll}\alpha & \alpha & 0 & 0 & 0\end{array}\right]^{T}$

where D represents the constitutive relations and $\boldsymbol{\alpha}$ represents the vector of linear thermal expansion coefficients.

By substituting Eq. (6) into Eq. (9), the stress components can be expressed as

$$
\begin{aligned}
& \sigma_{x^{\prime} x^{\prime}}=\frac{E}{1-v^{2}}\left[u_{, x^{\prime}}^{\prime}+z^{\prime} \theta_{y^{\prime}, x^{\prime}}+v\left(v_{, y^{\prime}}^{\prime}-z^{\prime} \theta_{x^{\prime}, y^{\prime}}\right)-(1+v) \alpha \Delta T\right] \\
& \sigma_{y^{\prime} y^{\prime}}=\frac{E}{1-v^{2}}\left[v_{, y^{\prime}}^{\prime}-z^{\prime} \theta_{x^{\prime}, y^{\prime}}+v\left(u_{, x^{\prime}}^{\prime}+z^{\prime} \theta_{y^{\prime}, x^{\prime}}\right)-(1+v) \alpha \Delta T\right]
\end{aligned}
$$




$$
\begin{aligned}
& \sigma_{x^{\prime} y^{\prime}}=\frac{E}{2(1+v)}\left[u_{, y^{\prime}}^{\prime}+v_{, x^{\prime}}^{\prime}+z^{\prime}\left(\theta_{y^{\prime}, y^{\prime}}-\theta_{x^{\prime}, x^{\prime}}\right)\right] \\
& \sigma_{x^{\prime} z^{\prime}}=\frac{E}{2(1+v)}\left[w_{, x^{\prime}}^{\prime}+\theta_{y^{\prime}}\right] \\
& \sigma_{y^{\prime} z^{\prime}}=\frac{E}{2(1+v)}\left[w_{, y^{\prime}}^{\prime}-\theta_{x^{\prime}}\right]
\end{aligned}
$$

\subsection{Strain Energy Density}

In linear elasticity, the strain energy density (SED) can be expressed as [35]

$W=\frac{1}{2}\left[\sigma_{x^{\prime} x^{\prime}} \varepsilon_{x^{\prime} x^{\prime}}^{m}+\sigma_{y^{\prime} y^{\prime}} \varepsilon_{y^{\prime} y^{\prime}}^{m}+\sigma_{z^{\prime} z^{\prime}} \varepsilon_{z^{\prime} z^{\prime}}^{m}+\sigma_{x^{\prime} y^{\prime}} \gamma_{x^{\prime} y^{\prime}}^{m}+\sigma_{x^{\prime} z^{\prime}} \gamma_{x^{\prime} z^{\prime}}^{m}+\sigma_{y^{\prime} z^{\prime}} \gamma_{y^{\prime} z^{\prime}}^{m}\right]$

By substituting the stress and mechanical strain components given in Eq. (10) and Eq. (8) into Eq. (11), the strain energy density can be obtained as

$$
\begin{aligned}
W=\frac{1}{2}\{ & \frac{E}{1-v^{2}}\left[u_{, x^{\prime}}^{\prime}+z^{\prime} \theta_{y^{\prime}, x^{\prime}}+v\left(v_{, y^{\prime}}^{\prime}-z^{\prime} \theta_{x^{\prime}, y^{\prime}}\right)-(1+v) \alpha . \Delta T\right]\left(u_{, x^{\prime}}^{\prime}+z^{\prime} \theta_{y^{\prime}, x^{\prime}}-\alpha \Delta T\right) \\
& +\frac{E}{1-v^{2}}\left[v_{, y^{\prime}}^{\prime}-z^{\prime} \theta_{x^{\prime}, y^{\prime}}+v\left(u_{, x^{\prime}}^{\prime}+z^{\prime} \theta_{y^{\prime}, x^{\prime}}\right)-(1+v) \alpha . \Delta T\right]\left(v_{, y^{\prime}}^{\prime}-z^{\prime} \theta_{x^{\prime}, y^{\prime}}-\alpha \Delta T\right) \\
& +\frac{E}{2(1+v)}\left[u_{, y^{\prime}}^{\prime}+v_{, x^{\prime}}^{\prime}+z^{\prime}\left(\theta_{y^{\prime}, y^{\prime}}-\theta_{x^{\prime}, x^{\prime}}\right)\right]^{2} \\
& \left.+\frac{E}{2(1+v)}\left[w_{, x^{\prime}}^{\prime}+\theta_{y^{\prime}}\right]^{2}+\frac{E}{2(1+v)}\left[w_{, y^{\prime}}^{\prime}-\theta_{x^{\prime}}\right]^{2}\right\}
\end{aligned}
$$

By integrating the SED given in Eq. (12) through the thickness of the shell, the strain energy per unit area, $\bar{W}$ can be obtained as

$$
\begin{aligned}
\bar{W} & =\int_{-h / 2}^{h / 2} W d z^{\prime}=\frac{E h}{2\left(1-v^{2}\right)}\left(u_{, x^{\prime}}^{\prime}+v_{, y^{\prime}}^{\prime}\right)^{2}+\frac{E h}{4(1+v)}\left[\left(u_{, y^{\prime}}^{\prime}+v_{, x^{\prime}}^{\prime}\right)^{2}-4 u_{, x^{\prime} v^{\prime}, y^{\prime}}^{\prime}\right] \\
& +\frac{E h}{1-v}\left[(\alpha \Delta T)^{2}-\alpha \Delta T\left(u_{, x^{\prime}}^{\prime}+v_{, y^{\prime}}^{\prime}\right)\right] \\
& +\frac{E h^{3}}{24\left(1-v^{2}\right)}\left[\left(\theta_{x^{\prime}, y^{\prime}}-\theta_{y^{\prime}, x^{\prime}}\right)^{2}+\frac{1-v}{2}\left(\left(\theta_{x^{\prime}, x^{\prime}}-\theta_{y^{\prime}, y^{\prime}}\right)^{2}+4 \theta_{y^{\prime}, x^{\prime}} \theta_{x^{\prime}, y^{\prime}}\right)\right] \\
& +\frac{k_{s} E h}{4(1+v)}\left[\left(w_{, x^{\prime}}^{\prime}+\theta_{y^{\prime}}\right)^{2}+\left(w_{, y^{\prime}}^{\prime}-\theta_{x^{\prime}}\right)^{2}\right]
\end{aligned}
$$

where $h$ represents the thickness of the flat shell, $v$ represents the Poisson's ratio, $E$ represents Young's modulus, $k_{s}=5 / 6$ represents the shear correction factor to account for the nonuniformity of the shear stresses [10]

The strain energy per unit area given in Eq. (13) can be represented as $\bar{W}=\bar{W}_{\text {inplane }}+\bar{W}_{\text {bending }}+\bar{W}_{\text {shear }}$

with 


$$
\begin{aligned}
\bar{W}_{\text {inplane }}= & \frac{E h}{2\left(1-v^{2}\right)}\left(u_{, x^{\prime}}^{\prime}+v_{, y^{\prime}}^{\prime}\right)^{2}+\frac{E h}{4(1+v)}\left[\left(u_{, y^{\prime}}^{\prime}+v_{, x^{\prime}}^{\prime}\right)^{2}-4 u_{, x^{\prime}}^{\prime} v_{, y^{\prime}}^{\prime}\right] \\
& +\frac{E h}{1-v}\left[(\alpha \Delta T)^{2}-\alpha \Delta T\left(u_{, x^{\prime}}^{\prime}+v_{, y^{\prime}}^{\prime}\right)\right] \\
\bar{W}_{\text {bending }}= & \frac{E h^{3}}{24\left(1-v^{2}\right)}\left[\left(\theta_{y^{\prime}, x^{\prime}}-\theta_{x^{\prime}, y^{\prime}}\right)^{2}+\frac{1-v}{2}\left(\left(\theta_{x^{\prime}, x^{\prime}}-\theta_{y^{\prime}, y^{\prime}}\right)^{2}+4 \theta_{y^{\prime}, x^{\prime}} \theta_{x^{\prime}, y^{\prime}}\right)\right] \\
\bar{W}_{\text {shear }}= & \frac{k_{s} E h}{4(1+v)}\left[\left(w_{, x^{\prime}}^{\prime}+\theta_{y^{\prime}}\right)^{2}+\left(w_{, y^{\prime}}^{\prime}-\theta_{x^{\prime}}\right)^{2}\right]
\end{aligned}
$$

where $\bar{W}_{\text {inplane }}, \bar{W}_{\text {bending }}, \bar{W}_{\text {shear }}$ represent the strain energy per unit area for in-plane, bending and shear deformations, respectively.

According to Mindlin-Reissner plate theory, plate element is developed with 5 degrees of freedom (DOF) as provided in Eq. (14). However, in order to simulate thin curved and stiffened structures 6 DOF shell elements are required. If the drilling rotation is not considered, all the resistance to the drilling rotation of each node comes directly from the coupling of other rotational DOFs of the non-planar surrounding nodes. When the model is discretized with very fine mesh, angles of the kinks between two elements, which are located next to each other, will become close to $2 \pi$ and the coupling effect is much reduced [7]. As a result, the global stiffness matrix may become singular and it results in unrealistic solution results. Therefore, in order to produce a small stiffness associated with drilling rotation, an additional fictitious torsional strain energy per unit area corresponding to the drilling rotation can be added as [7]:

$\bar{W}=\bar{W}_{\text {inplane }}+\bar{W}_{\text {bending }}+\bar{W}_{\text {shear }}+\bar{W}_{\text {torsional }}$

where

$$
\bar{W}_{\text {torsional }}=k_{T} \frac{E h}{2(1+v)}\left[\theta_{z^{\prime}}-\frac{1}{2}\left(v_{, x^{\prime}}^{\prime}-u_{, y^{\prime}}^{\prime}\right)\right]^{2}
$$

in which $k_{T}$ represents torsional constant [7].

Note that, the value of the fictitious stiffness needs to be small enough in order to ensure that this stiffness will not distort the global solution results [7].

\section{Kinematics of Flat Shells in Peridynamics}

Peridynamics (PD) is a new formulation of continuum theory which is first introduced by Silling [1]. In peridynamics, the motion of a particle is expressed by using integro-differential equations as

$$
\rho(\mathbf{x}) \ddot{\mathbf{u}}(\mathbf{x}, t)=\int_{H_{x}}\left(\mathbf{t}\left(\mathbf{u}^{\prime}-\mathbf{u}, \mathbf{x}^{\prime}-\mathbf{x}, t\right)-\mathbf{t}^{\prime}\left(\mathbf{u}-\mathbf{u}^{\prime}, \mathbf{x}-\mathbf{x}^{\prime}, t\right)\right) d V^{\prime}+\mathbf{b}(\mathbf{x}, t)
$$

which can also be represented in discrete form as [14]

$$
\rho_{(k)} \ddot{\mathbf{u}}_{(k)}=\sum_{j=1}^{N}\left(\mathbf{t}_{(k)(j)}\left(\mathbf{u}_{(j)}-\mathbf{u}_{(k)}, \mathbf{x}_{(j)}-\mathbf{x}_{(k)}, t\right)-\mathbf{t}_{(j)(k)}\left(\mathbf{u}_{(k)}-\mathbf{u}_{(j)}, \mathbf{x}_{(k)}-\mathbf{x}_{(j)}, t\right)\right) V_{(j)}+\mathbf{b}_{(k)}
$$

where $\rho$ represents the mass density, $\mathbf{u}$ and $\mathbf{b}$ represent displacement and body force vectors, respectively. As shown in Fig. 2, in PD, the motion of a material point is influenced by collective deformation of surrounding material points within a distance, $\delta$, which called horizon size. 
Material points within the horizon of a material point are called family members of that material point. In Eq. (16), $N$ represents the number of family members of material point $k, j$ represents the family member of material point $k, \mathbf{t}_{(k)(j)}$ represents the force density that material point $j$ exerts on material point $k$, and $\mathbf{t}_{(j)(k)}$ represents the force density that material point $k$ exerts on material point $j$.

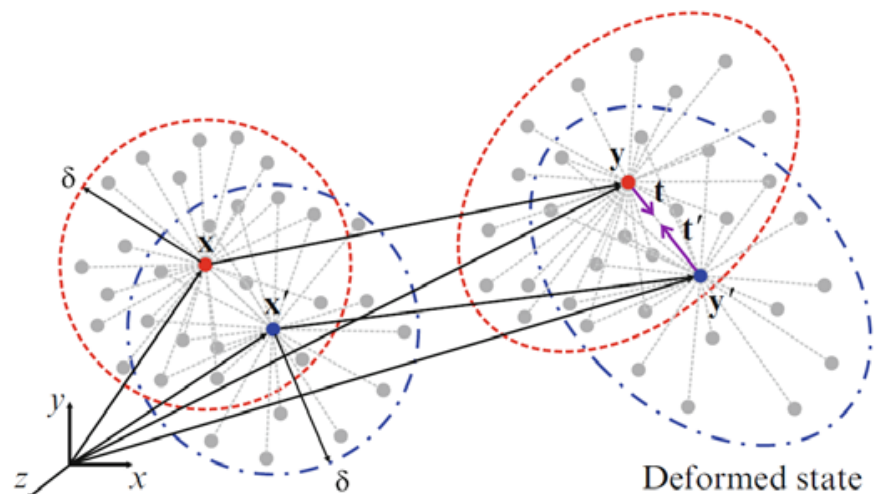

Undeformed state

Fig. 2. PD material points $\mathbf{x}_{(k)}$ and $\mathbf{x}_{(j)}$ influenced by the collective deformation of others in their families [14]

As explained by Madenci and Oterkus [14], the equation of motion can be derived based on the principle of virtual work by satisfying the Lagrange's equation as

$\frac{d}{d t}\left(\frac{\partial L}{\partial \dot{q}_{i}}\right)-\frac{\partial L}{\partial q_{i}}=0$

where $L$ represents the Lagrangian, $q_{i}$ represents the degree of freedom and $\dot{q}_{i}$ represents the time derivative of $q_{i}$. The Lagrangian function, $L$ can be expressed as

$L=T-U$

where $T$ and $U$ present kinetic and total potential energies.

In the following sections, first, the peridynamic form of strain energy density is established. Next, the kinetic and total potential energies are obtained. After obtaining the Lagrange function using Eq. (18), the equation of motions for shells are obtained by using Euler-Lagrange equation given in Eq. (17).

\subsection{Peridynamic Strain Energy Density}

Similar to the strain energy per unit area in classical continuum mechanics provided in Eq. (15a), the peridynamic form of strain energy per unit area for a flat shell can be presented as

$\bar{W}_{(k)}^{P D}=\bar{W}_{\text {inplane }(k)}^{P D}+\bar{W}_{\text {bending }(k)}^{P D}+\bar{W}_{\text {shear }(k)}^{P D}+\bar{W}_{\text {torsional }(k)}^{P D}$

where $\bar{W}_{\text {inplane }(k)}^{P D}, \bar{W}_{\text {bending }(k)}^{P D}, \bar{W}_{\text {shear }(k)}^{P D}$ represent the components of strain energy per unit area for inplane, bending, shear deformations, respectively. The term $\bar{W}_{\text {torsional }(k)}^{P D}$ represents the additional term that corresponds to drilling rotation. 
Similar to strain energy density definition by Madenci and Oterkus [14], the PD form of strain energy per unit area for in-plane deformations can be defined as

$\bar{W}_{\text {inplane }(k)}^{P D}=a_{i p 1} \vartheta_{(k)}^{2}-a_{i p 2} \vartheta_{(k)} \Delta T_{(k)}+a_{i p 3} \Delta T_{(k)}^{2}+b_{i p} \sum_{j=1}^{N}\left(s_{i p(k)(j)}-\alpha \Delta T_{(k)}\right)^{2} \xi V_{(j)}$

where $\vartheta_{(k)}$ represents the dilatation of material point $k, s_{i p(k)(j)}$ represents the relative bond stretch of in-plane displacement components between material point $k$ and material point $j$.

In Eq. (20), the terms $a_{i p 1}, a_{i p 2}, a_{i p 3}$, and $b_{i p}$ represent in-plane peridynamic constants as (Appendix A)

$a_{i p 1}=\frac{E h(3 v-1)}{4\left(1-v^{2}\right)}$

$a_{i p 2}=\frac{E h \alpha}{1-v^{2}}(3 v-1)=4 \alpha a_{i p 1}$

$a_{i p 3}=\frac{E h}{1-v^{2}}(3 v-1) \alpha^{2}=4 \alpha^{2} a_{i p 1}$

$b_{i p}=\frac{3}{\pi \delta^{3}} \frac{E}{1+v}$

The effect of temperature change on dilatation term can be included as [14, 28]

$\vartheta_{(k)}=d_{i p} \sum_{j=1}^{N}\left(s_{i p(k)(j)}-\alpha \Delta T_{(k)}\right) V_{(j)}+2 \alpha \Delta T_{(k)}$

where $d_{i p}$ represents the in-plane PD constant for dilatation as (Appendix A)

$d_{i p}=\frac{2}{\pi h \delta^{2}}$

The relative bond stretch for in-plane displacement components between two material points can be defined as [36]

$s_{i p(k)(j)}=\frac{\left(u_{(j)}^{\prime}-u_{(k)}^{\prime}\right) \cos \varphi+\left(v_{(j)}^{\prime}-v_{(k)}^{\prime}\right) \sin \varphi}{\xi}$

where $u_{(k)}^{\prime}, v_{(k)}^{\prime}$ and $u_{(j)}^{\prime}, v_{(j)}^{\prime}$ represent two in-plane degrees of freedom of material points $k$ and $j$, respectively. The parameter $\varphi$ represents the angle of interaction between material points $k$ and $j$ with respect to the local axis, $x^{\prime}$ in undeformed configuration as shown in Fig. 3. 


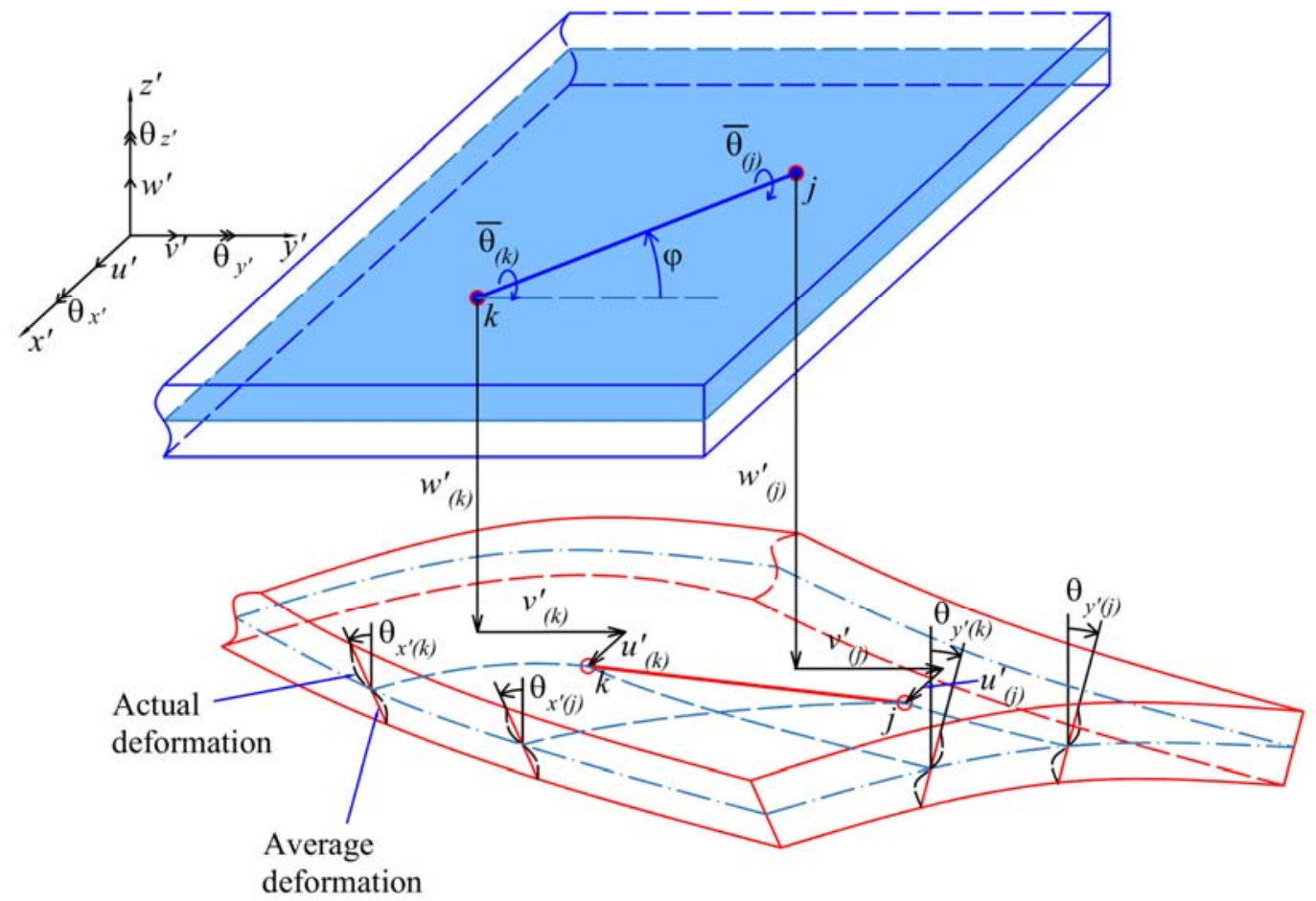

Fig. 3. Deformed and initial configuration of a flat shell in PD

The PD form of strain energy per unit area for bending deformations can be defined as $\bar{W}_{\text {bending }(k)}^{P D}=a_{b} \vartheta_{b(k)}^{2}+b_{b} \sum_{j=1}^{N} s_{b(k)(j)}^{2} \xi V_{(j)}$

where $a_{b}$ and $b_{b}$ represent PD constants for bending as (Appendix B)

$a_{b}=\frac{E h^{3}}{48} \frac{3 v-1}{1-v^{2}}$

$b_{b}=\frac{E h^{2}}{4(1+v) \pi \delta^{3}}$

The terms $\vartheta_{b(k)}$ and $s_{b(k)(j)}$ in Eq. (25) can be defined as

$\vartheta_{b(k)}=d_{b} \sum_{j=1}^{N} s_{b(k)(j)} V_{(j)}$

$s_{b(k)(j)}=\frac{-\left(\theta_{y^{\prime}(j)}-\theta_{y^{\prime}(k)}\right) \cos \varphi+\left(\theta_{x^{\prime}(j)}-\theta_{x^{\prime}(k)}\right) \sin \varphi}{\xi}$

with

$d_{b}=\frac{2}{\pi h \delta^{2}}$

Here, $\theta_{x^{\prime}}$ and $\theta_{y^{\prime}}$ represent rotational degrees of freedom with respect to $x^{\prime}$ and $y^{\prime}$ axes as shown in Fig. 1. 
The PD form of strain energy per unit area for shear deformations can be defined as

$\bar{W}_{\text {shear }(k)}^{P D}=\frac{1}{4} C_{s} \sum_{j=1}^{N}\left(\frac{w_{(j)}^{\prime}-w_{(k)}^{\prime}}{\xi}-\frac{\bar{\theta}_{(k)}+\bar{\theta}_{(j)}}{2}\right)^{2} \xi V_{(j)}$

where $C_{s}$ represents PD constant for the shear deformations as (Appendix C)

$C_{s}=\frac{3 k_{s}}{\pi \delta^{3}} \frac{E}{(1+v)}$

In Eq. (28) the terms $\bar{\theta}_{(k)}$ and $\bar{\theta}_{(j)}$ represent the rotations with respect to the line of action between the material points $k$ and $j$ as shown in Fig. 3. These rotations can be calculated as [18]

$\bar{\theta}_{(k)}=-\theta_{y^{\prime}(k)} \cos \varphi+\theta_{x^{\prime}(k)} \sin \varphi$

$\bar{\theta}_{(j)}=-\theta_{y^{\prime}(j)} \cos \varphi+\theta_{x^{\prime}(j)} \sin \varphi$

The torsional strain energy per unit area in PD can be defined as

$\bar{W}_{\text {torsional }(k)}^{P D}=\frac{1}{2} \sum_{j=1}^{N} C_{t}\left[\frac{\theta_{z^{\prime}(k)}+\theta_{z^{\prime}(j)}}{2}-\left(\frac{v_{(j)}^{\prime}-v_{(k)}^{\prime}}{\xi} \cos \varphi-\frac{u_{(j)}^{\prime}-u_{(k)}^{\prime}}{\xi} \sin \varphi\right)\right]^{2} \xi V_{(j)}$

where $\theta_{z^{\prime}(k)}$ and $\theta_{z^{\prime}(j)}$ represent drilling rotations, which are with respect to $z^{\prime}$ axis, of material points $k$ and $j$, respectively.

In Eq. (31), $C_{t}$ represents PD constant for torsional deformations as (Appendix D)

$C_{t}=k_{T} \frac{3}{2 \pi \delta^{3}} \frac{E}{1+v}$

As explained in Section. 2.4, the value of the fictitious stiffness corresponding to the drilling rotation needs to be small enough in order to ensure that this stiffness will not distort the global solution results. As suggested by Kanok-nukulchai [7], the value of torsional constant, $k_{T}$, can be chosen in order to make the torsional strain energy per unit area has the same order with the bending strain energy per unit area. Therefore, by equating Eq. (26b) and Eq. (32), the value of $k_{T}$ can be chosen as

$k_{T}=k_{0} \frac{h^{2}}{6}$

where $k_{0}$ constant is added for convergence in PD solution, which is inherited from the suggestion by Kanok-nukulchai [7] for convergence in FEA simulations, and it is chosen as $k_{0} \geq 1$.

Note that ordinary state based formulations for in-plane and bending strain energy as provided in Eq. (20) and (25) removed the limitation on Poisson's ratio.

3.2. PD Equations of Motion in the Local Coordinate System

The PD form of kinetic and total potential energies in Eq. (18) can be defined as

$$
\begin{aligned}
& T=\frac{1}{2} \sum_{k=1}^{N_{p}}\left(\rho h \dot{u}_{(k)}^{\prime 2}+\rho h v_{(k)}^{\prime 2}+\rho h w_{(k)}^{\prime 2}+\frac{\rho h^{3}}{12}\left(\dot{\theta}_{x^{\prime}(k)}^{2}+\dot{\theta}_{y^{\prime}(k)}^{2}\right)+\frac{\rho h I_{z^{\prime} z^{\prime}(k)}}{A_{(k)}} \dot{\theta}_{z^{\prime}(k)}^{2}\right) A_{(k)} \\
& U=\sum_{k=1}^{N_{p}}\left(\bar{W}_{(k)}^{P D}-\bar{b}_{x^{\prime}(k)} u_{(k)}^{\prime}-\bar{b}_{y^{\prime}(k)} v_{(k)}^{\prime}-\bar{b}_{z^{\prime}(k)} w_{(k)}^{\prime}-\bar{m}_{x^{\prime}(k)} \theta_{x^{\prime}(k)}-\bar{m}_{y^{\prime}(k)} \theta_{y^{\prime}(k)}\right) A_{(k)}
\end{aligned}
$$


where $N_{p}$ represents the total number of material points of the PD model, $\bar{b}_{x^{\prime}(k)}, \bar{b}_{y^{\prime}(k)}$ and $\bar{b}_{z^{\prime}(k)}$ represent applied forces per unit area at material point $k$ in $x^{\prime}, y^{\prime}$ and $z^{\prime}$ directions, respectively. The parameters $\bar{m}_{x^{\prime}(k)}$ and $\bar{m}_{y^{\prime}(k)}$ represent applied moments per unit area with respect to local coordinate $x^{\prime}$ and $y^{\prime}$, respectively. The parameter $I_{z^{\prime} z^{\prime}(k)}$ represents the moment of inertia with respect to $z^{\prime}$ axis. If shell is uniformly discretized with a grid size $\Delta x^{\prime}$, the moment of inertia, $I_{z^{\prime} z^{\prime}(k)}$ for each material point can be calculated as

$I_{z^{\prime} z^{\prime}(k)}=\frac{\left(\Delta x^{\prime}\right)^{4}}{6}$

By substituting the kinetic and total potential energies given in Eq. (34) into Eq. (18), the Lagrangian function is obtained. Subsequently, the equations of motion for flat shells can be obtained by using the Euler-Lagrange equation in Eq. (17) as

$$
\begin{aligned}
& \rho h \ddot{u}_{(k)}^{\prime}=\sum_{j=1}^{N}\left[\begin{array}{l}
2 a_{i p 1} d_{i p} \frac{1}{\xi}\left(\vartheta_{(k)}+\vartheta_{(j)}\right)-a_{i p 2} d_{i p} \frac{1}{\xi}\left(\Delta T_{(k)}+\Delta T_{(j)}\right) \\
+4 b_{i p}\left(s_{i p(k)(j)}-\alpha \Delta T_{(k)}\right)
\end{array}\right] \cos \varphi V_{(j)}+\bar{b}_{x^{\prime}(k)} \\
& \rho h \ddot{v}_{(k)}^{\prime}=\sum_{j=1}^{N}\left[\begin{array}{l}
2 a_{i p 1} d_{i p} \frac{1}{\xi}\left(\vartheta_{(k)}+\vartheta_{(j)}\right)-a_{i p 2} d_{i p} \frac{1}{\xi}\left(\Delta T_{(k)}+\Delta T_{(j)}\right) \\
+4 b_{i p}\left(s_{i p(k)(j)}-\alpha \Delta T_{(k)}\right)
\end{array}\right] \sin \varphi V_{(j)}+\bar{b}_{y^{\prime}(k)} \\
& \rho h \ddot{w}_{(k)}^{\prime}=C_{s} \sum_{j=1}^{N}\left\{\frac{w_{(j)}^{\prime}-w_{(k)}^{\prime}}{\xi}-\frac{1}{2}\left[-\left(\theta_{y^{\prime}(k)}+\theta_{y^{\prime}(j)}\right) \cos \varphi+\left(\theta_{x^{\prime}(k)}+\theta_{x^{\prime}(j)}\right) \sin \varphi\right]\right\} V_{(j)}+\bar{b}_{z^{\prime}(k)} \\
& \frac{\rho h^{3}}{12} \ddot{\theta}_{x^{\prime}(k)}=\sum_{j=1}^{N}\left[\frac{2 a_{b} d_{b}}{\xi}\left(\vartheta_{b(k)}+\vartheta_{b(j)}\right)+4 b_{b} s_{b(k)(j)}\right] \sin \varphi V_{(j)} \\
& +\frac{1}{2} C_{s} \sum_{j=1}^{N}\left\{\left(w_{(j)}^{\prime}-w_{(k)}^{\prime}\right) \sin \varphi-\frac{\xi}{2}\left[\begin{array}{l}
-\left(\theta_{y^{\prime}(k)}+\theta_{y^{\prime}(j)}\right) \sin \varphi \cos \varphi \\
+\left(\theta_{x^{\prime}(k)}+\theta_{x^{\prime}(j)}\right) \sin ^{2} \varphi
\end{array}\right]\right\} V_{(j)}+\bar{m}_{x^{\prime}(k)} \\
& \frac{\rho h^{3}}{12} \ddot{\theta}_{y^{\prime}(k)}=-\sum_{j=1}^{N}\left[\frac{2 a_{b} d_{b}}{\xi}\left(\vartheta_{b(k)}+\vartheta_{b(j)}\right)+4 b_{b} s_{b(k)(j)}\right] \cos \varphi V_{(j)} \\
& -\frac{1}{2} C_{s} \sum_{j=1}^{N}\left\{\left(w_{(j)}^{\prime}-w_{(k)}^{\prime}\right) \cos \varphi-\frac{\xi}{2}\left[\begin{array}{l}
-\left(\theta_{y^{\prime}(k)}+\theta_{y^{\prime}(j)}\right) \cos ^{2} \varphi \\
+\left(\theta_{x^{\prime}(k)}+\theta_{x^{\prime}(j)}\right) \sin \varphi \cos \varphi
\end{array}\right]\right\} V_{(j)}+\bar{m}_{y^{\prime}(k)} \\
& \frac{\rho h I_{z^{\prime} z^{\prime}(k)}}{A_{(k)}} \ddot{\theta}_{z^{\prime}(k)}=C_{t} \sum_{j=1}^{N}\left[\frac{\theta_{z^{\prime}(k)}+\theta_{z^{\prime}(j)}}{2}-\left(\frac{v_{(j)}^{\prime}-v_{(k)}^{\prime}}{\xi} \cos \varphi-\frac{u_{(j)}^{\prime}-u_{(k)}^{\prime}}{\xi} \sin \varphi\right)\right] \xi V_{(j)}
\end{aligned}
$$

The equation of motion in the local coordinate system given in Eq. (36) can be written in vector form as 
$\overline{\mathbf{m}}_{(k)}^{\prime} \ddot{\mathbf{i}}_{(k)}^{\prime}=\sum_{j=1}^{N} \mathbf{f}_{(k)(j)}^{\prime} V_{(j)}+\overline{\mathbf{b}}_{(k)}^{\prime}$

where

$\overline{\mathbf{m}}_{(k)}^{\prime}=\left[\begin{array}{cccccc}\rho h & 0 & 0 & 0 & 0 & 0 \\ 0 & \rho h & 0 & 0 & 0 & 0 \\ 0 & 0 & \rho h & 0 & 0 & 0 \\ 0 & 0 & 0 & \frac{\rho h^{3}}{12} & 0 & 0 \\ 0 & 0 & 0 & 0 & \frac{\rho h^{3}}{12} & 0 \\ 0 & 0 & 0 & 0 & 0 & \frac{\rho h I_{z^{\prime} z^{\prime}}}{A_{(k)}}\end{array}\right] ; \ddot{\mathbf{u}}_{(k)}^{\prime}=\left[\begin{array}{c}\ddot{u}_{(k)}^{\prime} \\ \ddot{v}_{(k)}^{\prime} \\ \dddot{w}_{(k)}^{\prime} \\ \ddot{\theta}_{x^{\prime}(k)} \\ \ddot{\theta}_{y^{\prime}(k)} \\ \ddot{\theta}_{z^{\prime}(k)}\end{array}\right] ; \overline{\mathbf{b}}_{(k)}^{\prime}=\left[\begin{array}{c}\bar{b}_{x^{\prime}(k)} \\ \bar{b}_{y^{\prime}(k)} \\ \bar{b}_{z^{\prime}(k)} \\ \bar{m}_{x^{\prime}(k)} \\ \bar{m}_{y^{\prime}(k)} \\ 0\end{array}\right]$

and

$\mathbf{f}_{(k)(j)}^{\prime}=\left[\begin{array}{llllll}f_{(k)(j)}^{u^{\prime}} & f_{(k)(j)}^{v^{\prime}} & f_{(k)(j)}^{w^{\prime}} & f_{(k)(j)}^{\theta_{x^{\prime}}} & f_{(k)(j)}^{\theta_{y^{\prime}}} & f_{(k)(j)}^{\theta_{\theta^{\prime}}}\end{array}\right]^{T}$

with

$$
\begin{aligned}
& f_{(k)(j)}^{u^{\prime}}= {\left[2 a_{i p 1} d_{i p} \frac{1}{\xi}\left(\vartheta_{(k)}+\vartheta_{(j)}\right)-a_{i p 2} d_{i p} \frac{1}{\xi}\left(\Delta T_{(k)}+\Delta T_{(j)}\right)+4 b_{i p}\left(s_{i p(k)(j)}-\alpha \Delta T_{(k)}\right)\right] \cos \varphi } \\
& f_{(k)(j)}^{v^{\prime}}= {\left[2 a_{i p 1} d_{i p} \frac{1}{\xi}\left(\vartheta_{(k)}+\vartheta_{(j)}\right)-a_{i p 2} d_{i p} \frac{1}{\xi}\left(\Delta T_{(k)}+\Delta T_{(j)}\right)+4 b_{i p}\left(s_{i p(k)(j)}-\alpha \Delta T_{(k)}\right)\right] \sin \varphi } \\
& f_{(k)(j)}^{w^{\prime}}= C_{s}\left\{\frac{w_{(j)}^{\prime}-w_{(k)}^{\prime}}{\xi}-\frac{1}{2}\left[-\left(\theta_{y^{\prime}(k)}+\theta_{y^{\prime}(j)}\right) \cos \varphi+\left(\theta_{x^{\prime}(k)}+\theta_{x^{\prime}(j)}\right) \sin \varphi\right]\right\} \\
& f_{(k)(j)}^{\theta_{x}^{\prime}}= {\left[\frac{2 a_{b} d_{b}}{\xi}\left(\vartheta_{b(k)}+\vartheta_{b(j)}\right)+4 b_{b} s_{b(k)(j)}\right] \sin \varphi } \\
&+\frac{1}{2} C_{s}\left\{\left(w_{(j)}^{\prime}-w_{(k)}^{\prime}\right) \sin \varphi-\frac{\xi}{2}\left[-\left(\theta_{y^{\prime}(k)}+\theta_{y^{\prime}(j)}\right) \sin \varphi \cos \varphi\right]+\left(\theta_{x^{\prime}(k)}+\theta_{x^{\prime}(j)}\right) \sin { }^{2} \varphi\right. \\
& f_{(k)(j)}^{\theta_{y}^{\prime}}=-\left[\frac{2 a_{b} d_{b}}{\xi}\left(\vartheta_{b(k)}+\vartheta_{b(j)}\right)+4 b_{b} s_{b(k)(j)}\right] \cos \varphi \\
&-\frac{1}{2} C_{s}\left\{\left(w_{(j)}^{\prime}-w_{(k)}^{\prime}\right) \cos \varphi-\frac{\xi}{2}\left[-\left(\theta_{y^{\prime}(k)}+\theta_{y^{\prime}(j)}\right) \cos { }^{2} \varphi\right.\right. \\
&\left.\left.+\left(\theta_{x^{\prime}(k)}+\theta_{x^{\prime}(j)}\right) \sin \varphi \cos \varphi\right]\right\} \\
& f_{(k)(j)}^{\theta_{z^{\prime}}}= C_{t}\left[\frac{\theta_{z^{\prime}(k)}+\theta_{z^{\prime}(j)}}{2}-\left(\frac{v_{(j)}^{\prime}-v_{(k)}^{\prime}}{\xi} \cos \varphi-\frac{u_{(j)}^{\prime}-u_{(k)}^{\prime}}{\xi} \sin \varphi\right)\right] \xi
\end{aligned}
$$

Note that the torsional strain energy per unit area is fictitious and it creates a small stiffness corresponding to drilling DOF. Therefore, the contribution of drilling rotation to in-plane displacements can be neglected in the equations of motion [7]. 
Note that the PD constants provided in Appendices A-D are obtained based on small deformation assumption. Therefore, the developed PD model is applicable for linear analysis of shell structures with small deformations. Within small deformation assumption, the balance laws such as balance of energy, linear momentum, angular momentum are satisfied.

\section{PD Equations of Motion in Global Coordinate System}

The equations of motion provided in Eq. (37) are derived in local or body-attached coordinates. For a structure which includes plates and shells joined together, the equations of motion for each material point needs to be transformed from local coordinates to global coordinates. Therefore, in this section, the transformation of equations of motion for flat and curved shells are presented.

\subsection{Coordinate Transformation}

As shown in Fig. 1, the local coordinates of a shell can be defined with respect to the global coordinates by unit vectors $\vec{n}_{x^{\prime}}, \vec{n}_{y^{\prime}}, \vec{n}_{z^{\prime}}$. The unit vectors for material point $k$ can be defined from the geometry of the shell as

$$
\begin{aligned}
& \vec{n}_{x^{\prime}(k)}=\left[\begin{array}{lll}
a_{1(k)} & a_{2(k)} & a_{3(k)}
\end{array}\right]^{T} \\
& \vec{n}_{y^{\prime}(k)}=\left[\begin{array}{lll}
b_{1(k)} & b_{2(k)} & b_{3(k)}
\end{array}\right]^{T} \\
& \vec{n}_{z^{\prime}(k)}=\left[\begin{array}{lll}
c_{1(k)} & c_{2(k)} & c_{3(k)}
\end{array}\right]^{T}
\end{aligned}
$$

The relationship between the global and local coordinates can be written as

$\mathbf{x}_{(k)}^{\prime}=\mathbf{H}_{(k)} \mathbf{x}_{(k)}$

where

$$
\begin{aligned}
\mathbf{x}_{(k)}^{\prime} & =\left[\begin{array}{lll}
x_{(k)}^{\prime} & y_{(k)}^{\prime} & z_{(k)}^{\prime}
\end{array}\right]^{T} \\
\mathbf{x}_{(k)} & =\left[\begin{array}{lll}
x_{(k)} & y_{(k)} & z_{(k)}
\end{array}\right]^{T} \\
\mathbf{H}_{(k)} & =\left[\begin{array}{lll}
\vec{n}_{x^{\prime}(k)} & \vec{n}_{y^{\prime}(k)} & \vec{n}_{z^{\prime}(k)}
\end{array}\right]^{T}=\left[\begin{array}{lll}
a_{1(k)} & a_{2(k)} & a_{3(k)} \\
b_{1(k)} & b_{2(k)} & b_{3(k)} \\
C_{1(k)} & c_{2(k)} & b_{3(k)}
\end{array}\right]
\end{aligned}
$$

The transformation of displacement vector can be defined as

$\mathbf{u}_{(k)}^{\prime}=\mathbf{T}_{(k)} \mathbf{u}_{(k)}$

with

$$
\begin{aligned}
& \mathbf{u}_{(k)}^{\prime}=\left[\begin{array}{llllll}
u_{(k)}^{\prime} & v_{(k)}^{\prime} & w_{(k)}^{\prime} & \theta_{x^{\prime}(k)} & \theta_{y^{\prime}(k)} & \theta_{z^{\prime}(k)}
\end{array}\right]^{T} \\
& \mathbf{u}_{(k)}=\left[\begin{array}{lllllll}
u_{(k)} & v_{(k)} & w_{(k)} & \theta_{x(k)} & \theta_{y(k)} & \theta_{z(k)}
\end{array}\right]^{T}
\end{aligned}
$$

where $\mathbf{u}_{(k)}^{\prime}$ and $\mathbf{u}_{(k)}$ represent displacement vectors in the local and global coordinate systems, respectively. The parameter $\mathbf{T}_{(k)}$ represents the displacement transformation matrix of material point $k$, which can be obtained from the coordinate transformation matrix, $\mathbf{H}_{(k)}$ as

$$
\mathbf{T}_{(k)}=\left[\begin{array}{cc}
\mathbf{H}_{(k)} & \mathbf{0} \\
\mathbf{0} & \mathbf{H}_{(k)}
\end{array}\right]
$$


4.2. Transformation of Equations of Motion for Flat Shells

By using the relations given in Eq. (40a), the equations of motion for shell given in Eq. (37a) can be written as

$$
\overline{\mathbf{m}}_{(k)}^{\prime} \mathbf{T}_{(k)} \ddot{\mathbf{u}}_{(k)}=\sum_{j=1}^{N} \mathbf{f}_{(k)(j)}^{\prime} V_{(j)}+\overline{\mathbf{b}}_{(k)}^{\prime}
$$

Multiplying both sides by $\mathbf{T}_{(k)}^{T}$ results in

$$
\mathbf{T}_{(k)}^{T} \overline{\mathbf{m}}_{(k)}^{\prime} \mathbf{T}_{(k)} \ddot{\mathbf{i}}_{(k)}=\mathbf{T}_{(k)}^{T}\left(\sum_{j=1}^{N} \mathbf{f}_{(k)(j)}^{\prime} V_{(j)}+\overline{\mathbf{b}}_{(k)}^{\prime}\right)
$$

As a result, the equation of motion in global coordinates becomes

$$
\overline{\mathbf{m}}_{(k)} \ddot{\mathbf{u}}_{(k)}=\sum_{j=1}^{N} \mathbf{f}_{(k)(j)} V_{(j)}+\overline{\mathbf{b}}_{(k)}
$$

where

$$
\begin{aligned}
& \mathbf{f}_{(k)(j)}=\mathbf{T}_{(k)}^{T} \mathbf{f}_{(k)(j)}^{\prime} \\
& \overline{\mathbf{b}}_{(k)}=\mathbf{T}_{(k)}^{T} \overline{\mathbf{b}}_{(k)}^{\prime} \\
& \overline{\mathbf{m}}_{(k)}=\mathbf{T}_{(k)}^{T} \overline{\mathbf{m}}_{(k)}^{\prime} \mathbf{T}_{(k)}
\end{aligned}
$$

\subsection{Transformation of Equations of Motion for Curved Shells}

In general, curved shells can be analysed by dividing them into suitable number of flat shell elements [37]. Each flat shell element can be considered as a material point with kinematics described in Section 3 with the orientation represented by three unit vectors given in Eq. (38). Therefore, the equations of motions for each material point need to be transformed to global coordinate system. Since each material point has different displacement transformation matrix, the equations of motion for curved shells can be obtained by modifying Eq. (43) as

$$
\mathbf{T}_{(k)(j)}^{T} \overline{\mathbf{m}}_{(k)}^{\prime} \mathbf{T}_{(k)(j)} \ddot{\mathbf{u}}_{(k)}=\mathbf{T}_{(k)(j)}^{T}\left(\sum_{j=1}^{N} \mathbf{f}_{(k)(j)}^{\prime} V_{(j)}+\overline{\mathbf{b}}_{(k)}^{\prime}\right)
$$

where $\mathbf{T}_{(k)(j)}$ represent the transformation matrix of interaction between material point $k$ and $j$ and it can be defined as [21]

$$
\mathbf{T}_{(k)(j)}=\left[\begin{array}{cc}
\mathbf{H}_{(k)(j)} & \mathbf{0} \\
\mathbf{0} & \mathbf{H}_{(k)(j)}
\end{array}\right]
$$

with

$$
\mathbf{H}_{(k)(j)}=\left[\begin{array}{lll}
a_{1(k)(j)} & a_{2(k)(j)} & a_{3(k)(j)} \\
b_{1(k)(j)} & b_{2(k)(j)} & b_{3(k)(j)} \\
c_{1(k)(j)} & c_{2(k)(j)} & b_{3(k)(j)}
\end{array}\right]
$$

or

$$
\mathbf{H}_{(k)(j)}=\left[\begin{array}{lll}
\vec{n}_{x^{\prime}(k)(j)} & \vec{n}_{y^{\prime}(k)(j)} & \vec{n}_{z^{\prime}(k)(j)}
\end{array}\right]^{T}
$$

where $\vec{n}_{x^{\prime}(k)(j)}, \vec{n}_{y^{\prime}(k)(j)}, \vec{n}_{z^{\prime}(k)(j)}$ can be represented as [21] 


$$
\begin{aligned}
& \vec{n}_{x^{\prime}(k)(j)}=\frac{\left(\vec{n}_{x^{\prime}(k)}+\vec{n}_{x^{\prime}(j)}\right)}{\left|\left(\vec{n}_{x^{\prime}(k)}+\vec{n}_{x^{\prime}(j)}\right)\right|} \\
& \vec{n}_{y^{\prime}(k)(j)}=\frac{\left(\vec{n}_{y^{\prime}(k)}+\vec{n}_{y^{\prime}(j)}\right)}{\left|\left(\vec{n}_{y^{\prime}(k)}+\vec{n}_{y^{\prime}(j)}\right)\right|} \\
& \vec{n}_{z^{\prime}(k)(j)}=\frac{\left(\vec{n}_{z^{\prime}(k)}+\vec{n}_{z^{\prime}(j)}\right)}{\left|\left(\vec{n}_{z^{\prime}(k)}+\vec{n}_{z^{\prime}(j)}\right)\right|}
\end{aligned}
$$

\section{Damage Prediction in Peridynamics}

In this section, a PD damage criterion based on critical energy release rate is introduced. The energy release rate for each interaction is calculated and compared with the critical value. If the energy release rate exceeds the critical value, the interaction is considered as broken. When the interaction between two material points is broken, the force densities between these points are irreversibly removed which leads to crack growth. The state of interaction, intact or broken, can be represented by a function, $\mu_{(k)(j)}$ introduced by Silling and Askari [2] as

$\mu_{(k)(j)}\left(\mathbf{x}_{(j)}-\mathbf{x}_{(k)}, t\right)=\left\{\begin{array}{l}1 \text { if interaction exists, } \\ 0 \text { if no interaction. }\end{array}\right.$

By including function $\mu_{(k)(j)}$, the PD equations of motion given in Eq. (45a) can be written as

$$
\left[\mathbf{T}_{(k)(j)}^{T} \overline{\mathbf{m}}_{(k)}^{\prime} \mathbf{T}_{(k)(j)}\right] \ddot{\mathbf{u}}_{(k)}=\sum_{j=1}^{N}\left[\mathbf{T}_{(k)(j)}^{T} \mu_{(k)(j)} \mathbf{f}_{(k)(j)}^{\prime}\right] V_{(j)}+\overline{\mathbf{b}}_{(k)}
$$

Note that, by including state of interaction represented by function $\mu_{(k)(j)}$, the dilatation, $\vartheta_{(k)}$, given in Eq. (22), and the term, $\vartheta_{b(k)}$, given in Eq. (27a) can be rewritten as

$$
\begin{aligned}
& \vartheta_{(k)}=d_{i p} \sum_{j=1}^{N} \mu_{(k)(j)}\left(s_{i p(k)(j)}-\alpha \Delta T_{(k)}\right) V_{(j)}+2 \alpha \Delta T_{(k)} \\
& \vartheta_{b(k)}=d_{b} \sum_{j=1}^{N} \mu_{(k)(j)} s_{b(k)(j)} V_{(j)}
\end{aligned}
$$

In order to represent the level of damage on the structure, the local damage, $\phi$, which is the ratio of eliminated interactions to the total number of interactions associated with a material point within its horizon is introduced as [2]

$$
\phi\left(\mathbf{x}_{(k)}, t\right)=1-\frac{\sum_{j=1}^{N} \mu_{(k)(j)}\left(\mathbf{x}_{(j)}-\mathbf{x}_{(k)}, t\right) V_{(j)}}{\sum_{j=1}^{N} V_{(j)}}
$$

In order to decide the state of interaction as described in Eq. (47), a damage criterion needs to be introduced. It can be represented by either critical bond stretch [1] or critical energy release rate $[27,28]$. In this study, the damage criteria based on the critical energy release rate is used. The average critical energy release rate, $g_{c}$, for one interaction can be computed by the relation suggested by Madenci and Oterkus [27, 28] as 
$g_{c}=\frac{G_{c}}{N_{c}}$

where $G_{c}$ represents the critical energy release rate of material and $N_{c}$ represents the total number of interactions passing through a unit crack area, $A$. This number can be defined by counting the interactions passing through a unit crack surface in which the interaction passing the crack tip can be counted as $1 / 2$ interaction. Fig. 4 shows the interactions passing a unit crack surface $A$. As shown in the figure, for a horizon size $\delta=3.015 \Delta x$, there are totally 48 interactions passing the unit crack surface, in which 24 interactions passing the crack tips. Therefore, the total number of interactions passing the unit crack surface can be counted as $N_{c}=36$.
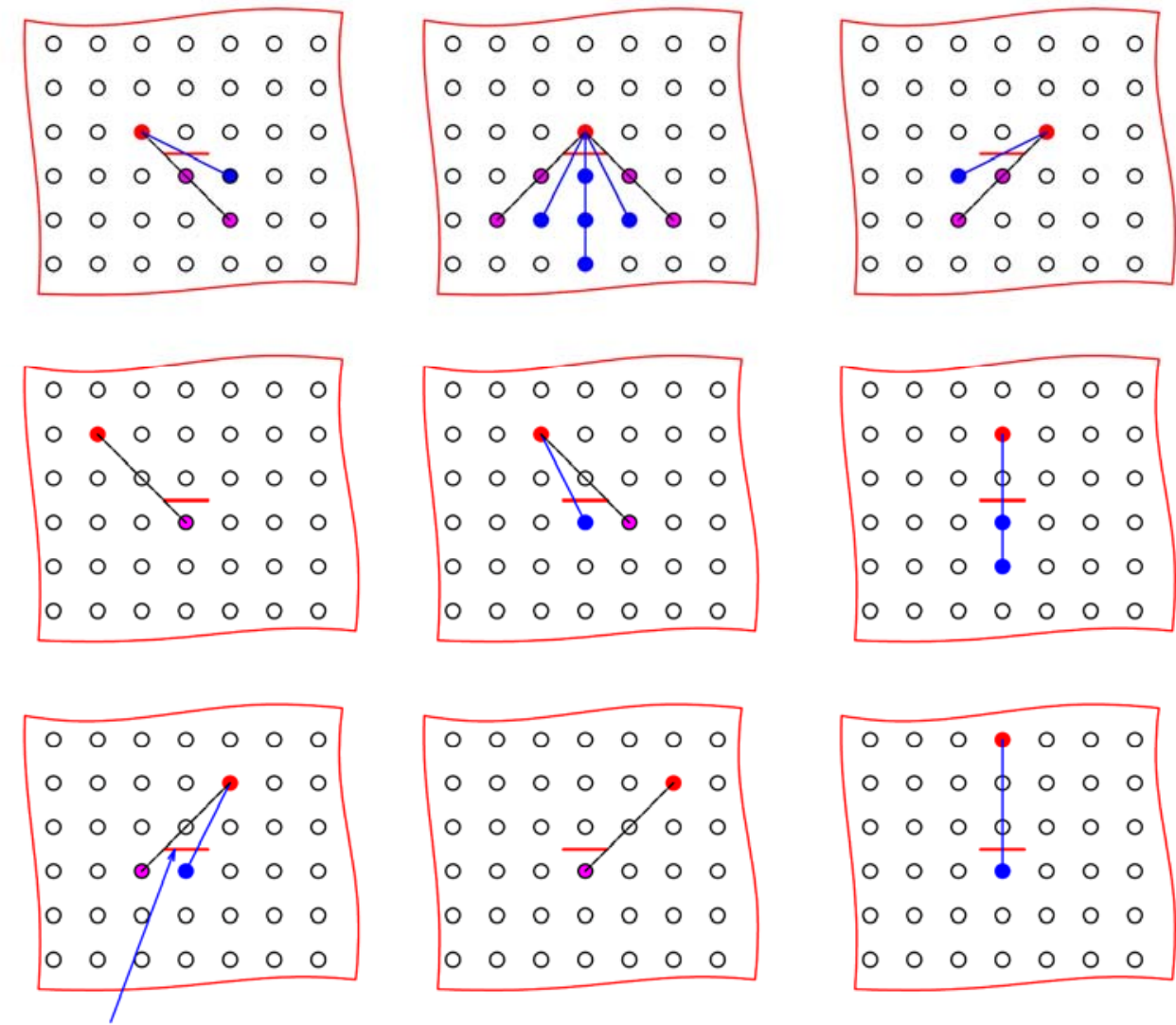

Crack surface, $A$

Interaction is counted as 1 interaction

Interaction is counted as $1 / 2$ interaction

Fig. 4. Counting the number of interaction, $N_{c}$, passing unit crack surface $[27,28]$

The damage criteria in peridynamics can be described as

$\bar{g}_{(k)(j)}<g_{c} \rightarrow$ interaction exists: $\mu_{(k)(j)}=1$

$\bar{g}_{(k)(j)} \geq g_{c} \rightarrow$ interaction is broken: $\mu_{(k)(j)}=0$

where $\bar{g}_{(k)(j)}$ represents the energy release rate for interaction between material points $k$ and $j$ which can be calculated as 
$\bar{g}_{(k)(j)}=\frac{1}{2}\left(g_{(k)(j)}+g_{(j)(k)}\right)$

with

$g_{(k)(j)}=\frac{1}{(\Delta x) h} \omega_{(k)(j)} V_{(k)} V_{(j)}$

$g_{(j)(k)}=\frac{1}{(\Delta x) h} \omega_{(j)(k)} V_{(j)} V_{(k)}$

where $\omega_{(k)(j)}$ and $\omega_{(j)(k)}$ represent micropotentials of the interaction between material point $k$ and $j$. The micropotential $\omega_{(k)(j)}$ can be calculated as

$\omega_{(k)(j)}=\omega_{\text {inplane }(k)(j)}+\omega_{\text {shear }(k)(j)}+\omega_{\text {bending }(k)(j)}$

where $\omega_{\text {inplane }(k)(j)}, \omega_{\text {shear }(k)(j)}, \omega_{\text {bending }(k)(j)}$ represent micropotentials for in-plane, shear, and bending deformations, respectively. Note that the contribution of drilling rotation is not included in the calculation of total micropotential in Eq. (55) because the strain energy density caused by this DOF is fictitious.

In linear elasticity with small deformation, the relationship between relative bond stretch and force density is linear. Therefore, by applying the same idea introduced by Madenci and Oterkus [27, 28], the in-plane micropotential $\omega_{\text {inplane }(k)(j)}$ can be calculated as

$\omega_{\text {inplane }(k)(j)}=\frac{1}{2 h} t_{i p(k)(j)} \xi\left(s_{i p(k)(j)}-\alpha \Delta T_{(k)}\right)$

where

$t_{i p(k)(j)}=\left[\frac{2 a_{i p 1} d_{i p}}{\xi} \vartheta_{(k)}-\frac{a_{i p 2} d_{i p}}{\xi} \Delta T_{(k)}+2 b_{i p}\left(s_{i p(k)(j)}-\alpha \Delta T_{(k)}\right)\right]$

The bending micropotential $\omega_{\text {bending(k)(j) }}$ can be calculated as

$\omega_{\text {bending }(k)(j)}=\frac{1}{2 h} t_{b(k)(j)} \xi_{s_{b(k)(j)}}$

where

$t_{b(k)(j)}=\left[\frac{2 a_{b} d_{b}}{\xi} \vartheta_{b(k)}+2 b_{b} s_{b(k)(j)}\right]+\frac{1}{4} C_{s}\left\{\left(w_{(j)}^{\prime}-w_{(k)}^{\prime}\right)-\frac{\xi}{2}\left[\begin{array}{c}-\left(\theta_{y^{\prime}(k)}+\theta_{y^{\prime}(j)}\right) \cos \varphi \\ +\left(\theta_{x^{\prime}(k)}+\theta_{x^{\prime}(j)}\right) \sin \varphi\end{array}\right]\right\}$

The shear micropotential $\omega_{\text {shear }(k)(j)}$ can be calculated as

$\omega_{\text {shear }(k)(j)}=\frac{1}{2 h} t_{\text {shear }(k)(j)} \xi_{\text {shear }(k)(j)}$

where

$t_{\text {shear }(k)(j)}=\frac{1}{2} f_{(k)(j)}^{w^{\prime}}=\frac{1}{2} C_{s}\left\{\frac{w_{(j)}^{\prime}-w_{(k)}^{\prime}}{\xi}-\frac{1}{2}\left[-\left(\theta_{y^{\prime}(k)}+\theta_{y^{\prime}(j)}\right) \cos \varphi+\left(\theta_{x^{\prime}(k)}+\theta_{x^{\prime}(j)}\right) \sin \varphi\right]\right\}$

$S_{\text {shear }(k)(j)}=\frac{w_{(j)}^{\prime}-w_{(k)}^{\prime}}{\xi}$ 


\section{Numerical implementation}

The equation of motion in PD can be solved by using a meshless scheme. The domain is divided into a uniform mesh, with material points associated with specific volumes. The numerical procedure in PD predictions for shell structures is shown in Fig 5. 


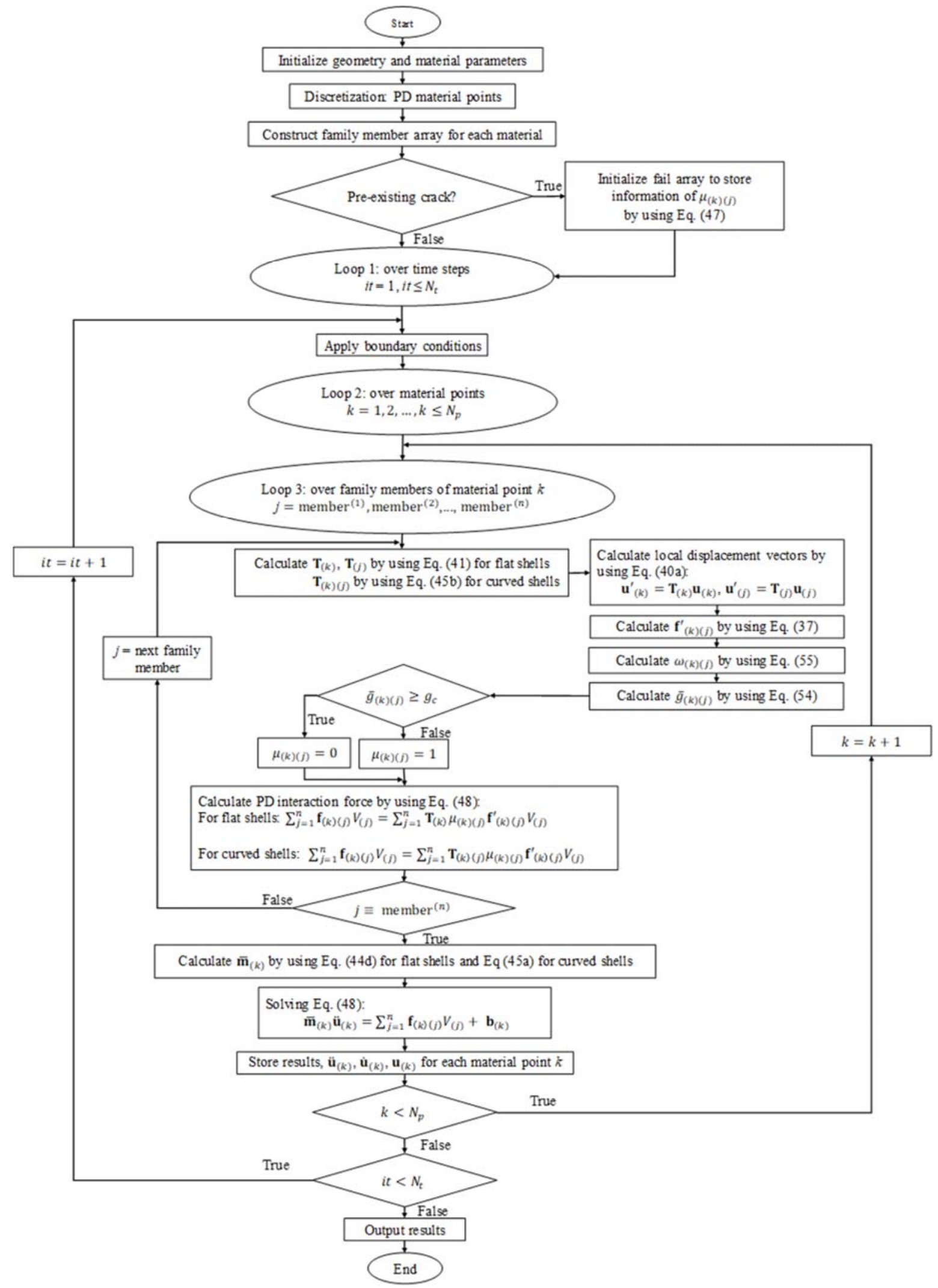

Fig. 5. Numerical procedure 
For stiffened structures, the geometrical and material properties as well as family members for each material point need to be defined. Depending on the location of the material point, different procedure is used. As an example, the stiffened structure shown in Fig. 6(a) is considered. Fig. $6(\mathrm{~b})$ demonstrates the model discretization for a stiffened structure with three shells $S_{A}, S_{B}, S_{C}$. In Fig. 6(a) lines $L_{1}, L_{2}, L_{3}$ represent the intersections between shells and point $k$ represents the intersection of the three shells. The material points located at the intersection lines are identified such as $\left(L_{1 A}, L_{1 B}\right),\left(L_{2 A}, L_{2 C}\right),\left(L_{3 B}, L_{3 C}\right)$ as shown in Fig. 6(b). During the discretization, material points along lines $L_{1 B}, L_{2 C}, L_{3 C}$ are removed, material points along lines $L_{1 A}, L_{2 A}, L_{3 B}$ are set as joint points and the material point $k$ is set as the intersection point of three shells as shown in Fig. $6(b)$.

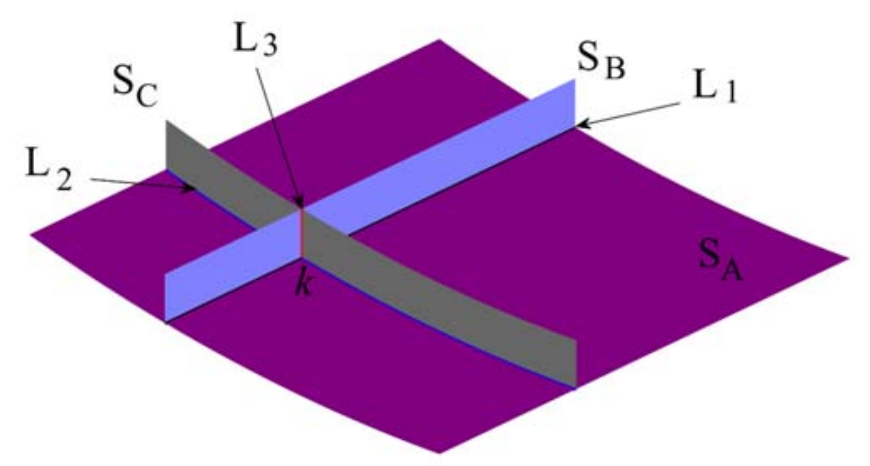

(a)

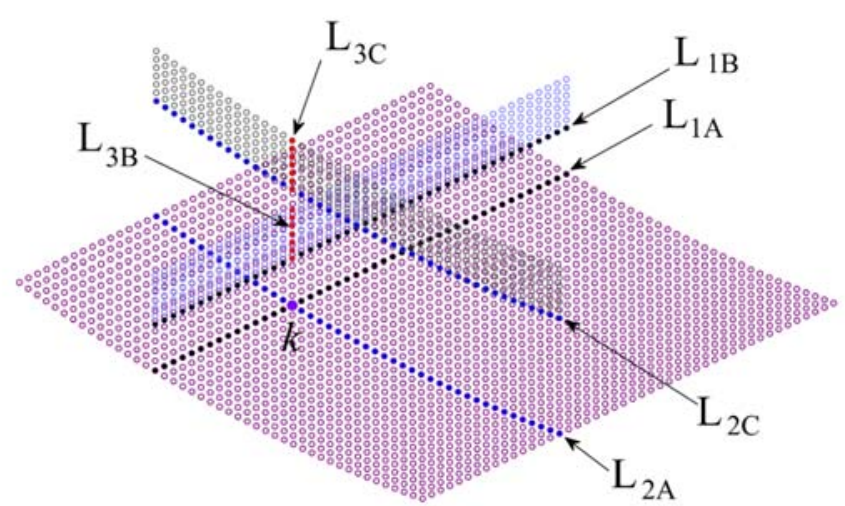

(b)

Fig. 6. Stiffened structue (a) geometry, (b) model discretization

Fig. 7 represents the family members for material points at two different locations. As shown in Fig. 7(a), material point 8 is located on shell $S_{B}$, therefore the family members of this material point belong to shell $S_{B}$. On the other hand, material point 4 is located at the intersection of shell 
$S_{A}$ and $S_{B}$ as shown in Fig. 7(b), therefore its family members belong to both shells. As a demonstration, Fig. 8 shows the number of family members for a stiffened structure.

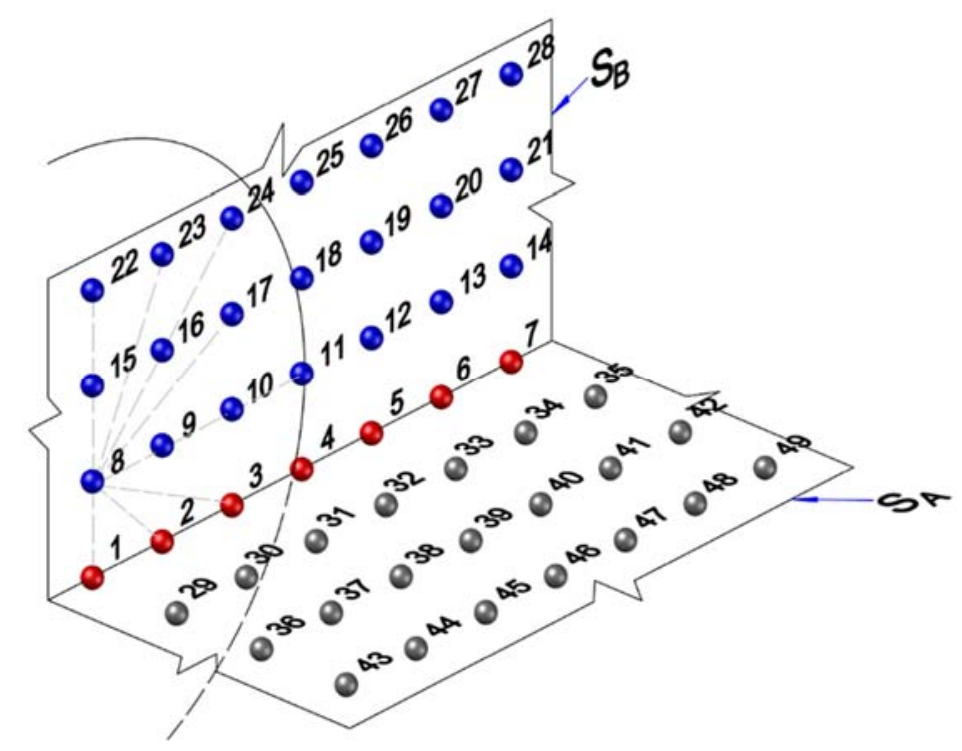

(a)

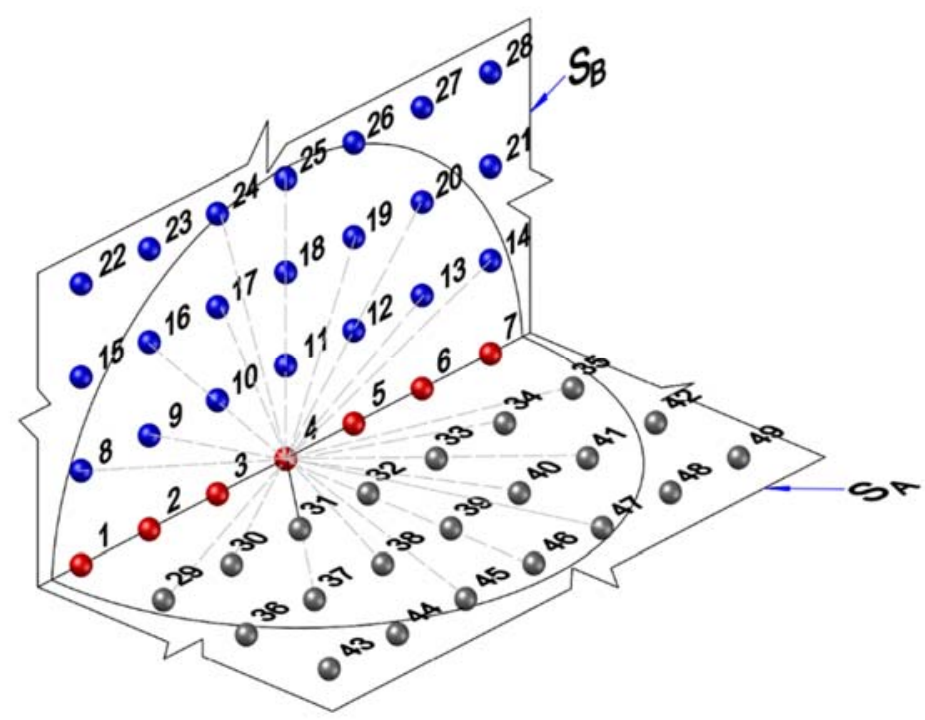

(b)

Fig. 7. Identification of family members for a material point located (a) on the shell (b) at the intersection

For a stiffened structure, each shell may have different material and geometrical properties. The material and geometrical properties of each interaction is determined based on the family member of each material point. As shown in Fig. 7(a), since material point 8 and its family members are located on shell $S_{B}$, the material and geometrical properties of material point 8 are obtained from 
shell $S_{B}$. On the other hand, as shown in Fig. 7(b) material point 4 and its family members are located at both shells $S_{A}$ and $S_{B}$. Therefore, material and geometrical properties are determined based on the interaction between material points. For the interaction between material points 4 and 10 , since the neighbour material point 10 belongs to shell $S_{B}$, the material and geometrical properties of the interaction is included for shell $S_{B}$. Similarly, for the interaction between material points 4 and 31 , since the neighbour material point 31 belongs to shell $S_{A}$, the material and geometrical properties of the interaction is included for shell $S_{A}$. Material points 4 and 5 are located at the interface. Therefore, the interaction forces between these points are calculated by summing two interaction forces. Each interaction force in shell's local coordinate system is calculated by using Eq. (36) and transformed to global coordinate by using the transformation matrix given in Eq. (41) or Eq. (45b).

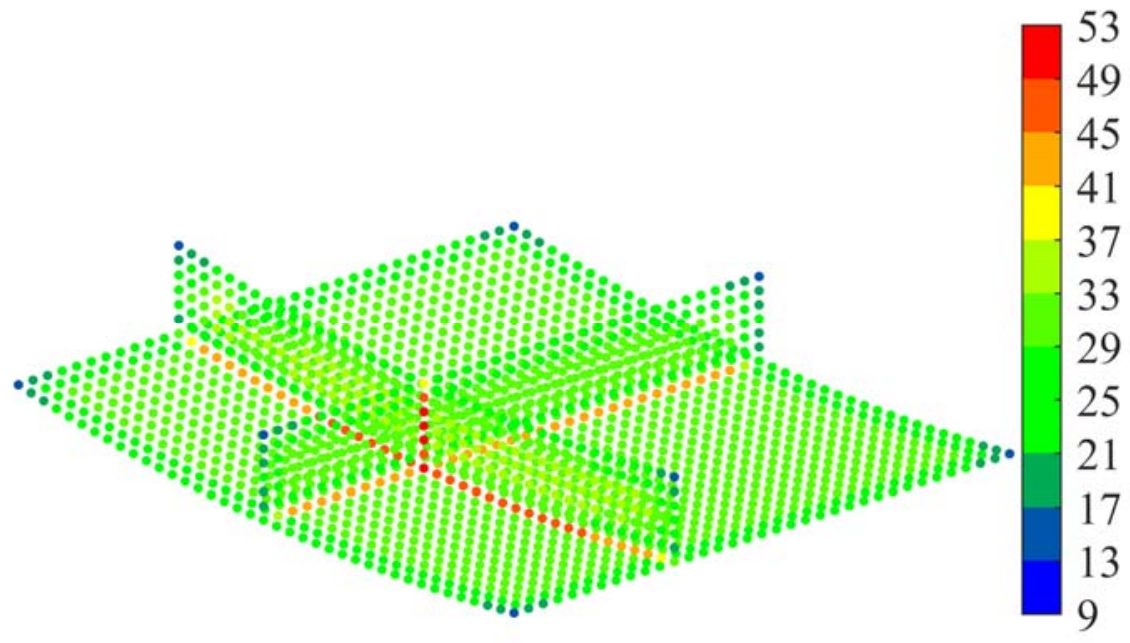

Fig. 8. Number of family members of material points on a stiffened structure

\section{Numerical results}

For verification purposes, the proposed PD model is compared against FEA solutions. The integration technique for static analyses is described in Appendix E, whereas the integration technique used for dynamic analysis is presented in [14]. The FEA solutions are conducted by using ANSYS commercial software by using SHELL181 element. In PD theory, the boundary conditions can be implemented through fictitious layers as described by Macek and Silling [38]. Based on numerical experiments, in order to ensure that the imposed boundary condition is accurately reflected in the real domain, this layer needs to be at least at the size of the horizon, $\delta$.

\subsection{A flat shell subjected to constant static loading}

In order to verify the developed PD model for flat shells, a square flat shell with dimensions $L=W=1 \mathrm{~m}$, and thickness $h=0.1 \mathrm{~m}$ is investigated as shown in Fig. 9(a). The flat shell is fixed on the left end and subjected to uniformly distributed loading along the right edge in both $x$ and negative $z$ directions as $p_{x}=1 \times 10^{7} \mathrm{~N} / \mathrm{m}, p_{z}=-1 \times 10^{7} \mathrm{~N} / \mathrm{m}$, respectively. The material has Young's modulus of $E=2 \times 10^{11} \mathrm{~N} / \mathrm{m}^{2}$ and Poisson's ratio of $v=0.45$. 
In PD model, the shell is discretized with uniform 150 integration points along each direction. As shown in Fig. 9(b), in order to apply boundary conditions along the left edge, fictitious layers of material points, shown in black, are added on the left edge. All DOFs of these fictitious points are set equal to zero. In FEA model, same mesh size is also used.

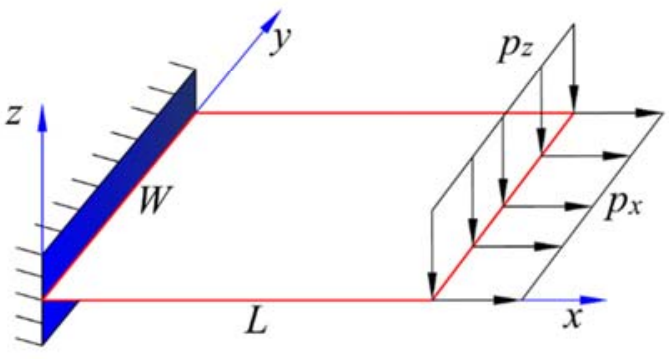

(a)

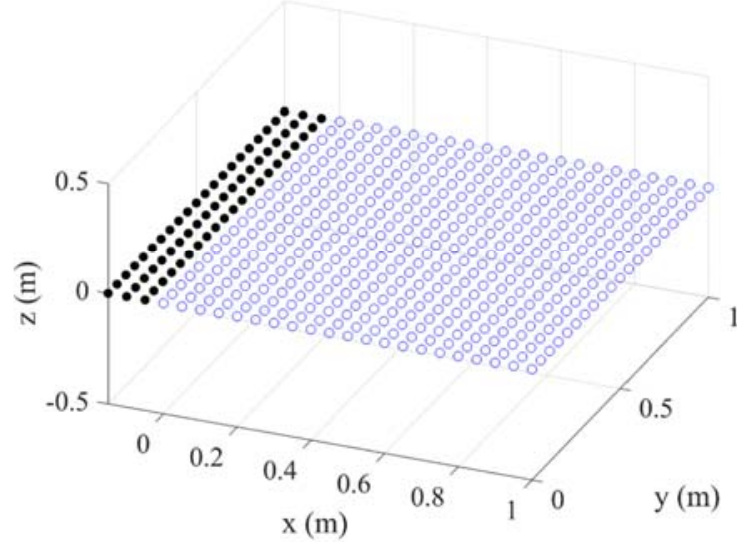

(b)

Fig. 9. Flat shell subjected to static constant loading (a) geometry, (b) model discretization As given in Eq. (32) and (33), the PD constant for torsional deformations depends on $k_{0}$ which is added for convergence in PD solutions. Therefore, the effect of $k_{0}$ to the PD solution is investigated as shown in Fig. 10 with horizon size $\delta=3.015 \Delta x$. As can be seen from the figure, the PD results for displacements and rotations of the flat shell is stable for various value of $k_{0}$. After checking the stability of the PD solutions, $k_{0}=1$ is chosen.

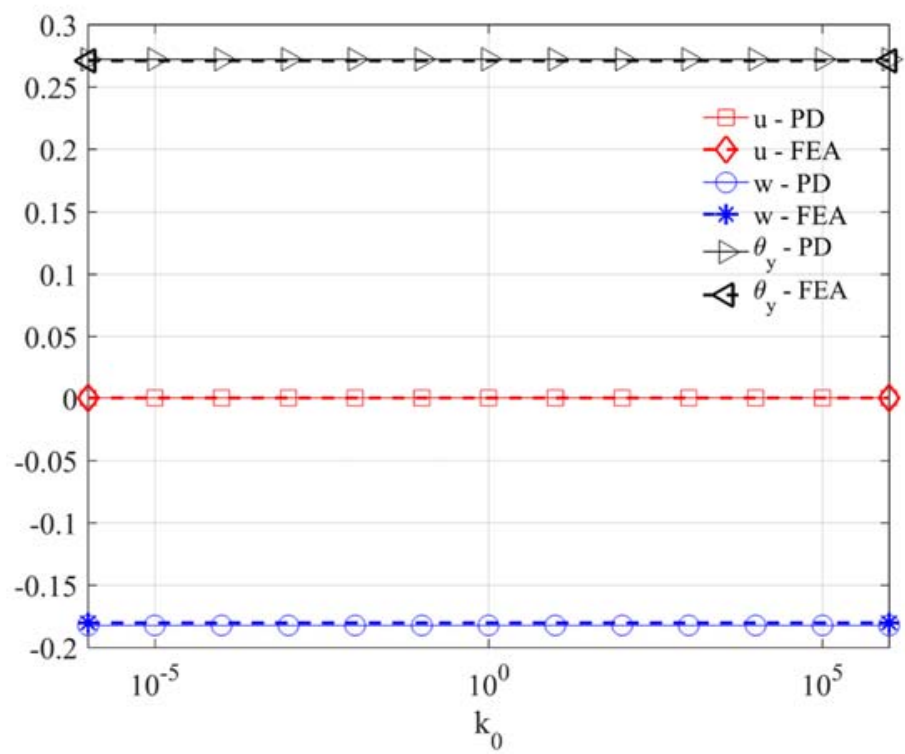

Fig. 10. Effect of torsional coefficient $k_{0}$ on PD results at $(x=L, y=W / 2)$, horizon size $\delta=3.015 \Delta x$. 
The effect of horizon size on PD results are investigated by comparing with FEA solutions. Fig. 11 shows the variation of relative error between PD and ANSYS predictions. The relative error for each DOF is calculated as

$\% \operatorname{error}(q)=\frac{\left|q^{F E A}-q^{P D}\right|}{\left|q^{F E A}\right|} \times 100$

where $q^{F E A}$ represents the FEA and $q^{P D}$ represents the PD solution for a degree of freedom, $q$.

As it can be seen from Fig.11, relative error between PD and ANSYS predictions reduces as the horizon size increases. When the horizon size is bigger than $\delta=3 \Delta x$, the relative error for all degrees of freedom is less than $3 \%$. Therefore, to reduce the computational time $\delta=3.015 \Delta x$ is chosen. Note that, the extra value $0.015 \Delta x$ is added into horizon size in order to ensure that all material points within a distance of $3 \Delta x$ is included.

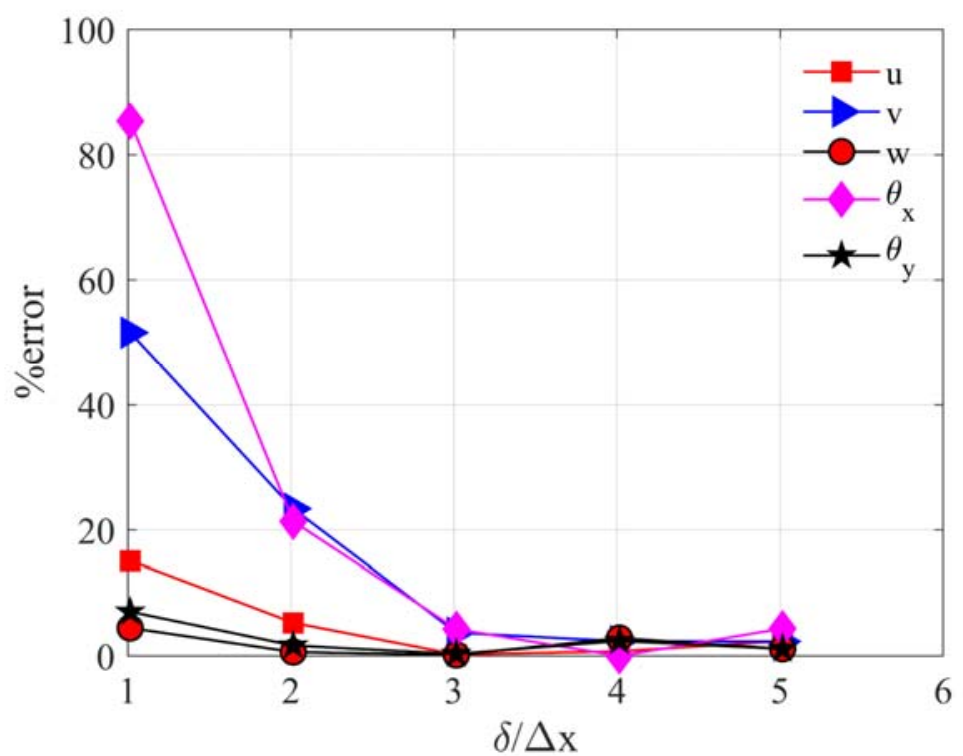

Fig. 11. Effect of horizon size on PD prediction results at $(x=L, y=3 W / 4)$

Figs. 12-17 present the comparison of PD and ANSYS predictions for 6 DOFs with $\delta=3.015 \Delta x$ and $k_{0}=1$. As it can be seen from the figures, PD predictions agree very well with FEA results. Moreover, the good agreement of drilling rotation, $\theta_{z}$ shows that the additional PD equation of motion for drilling rotation is acceptable. 


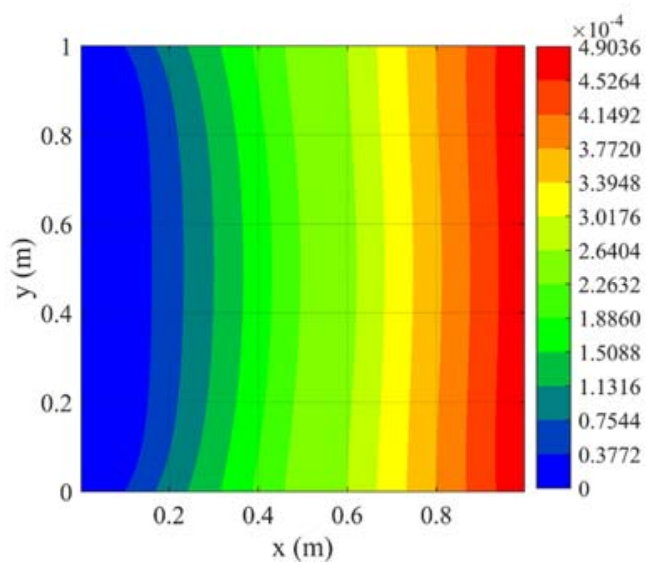

(a)

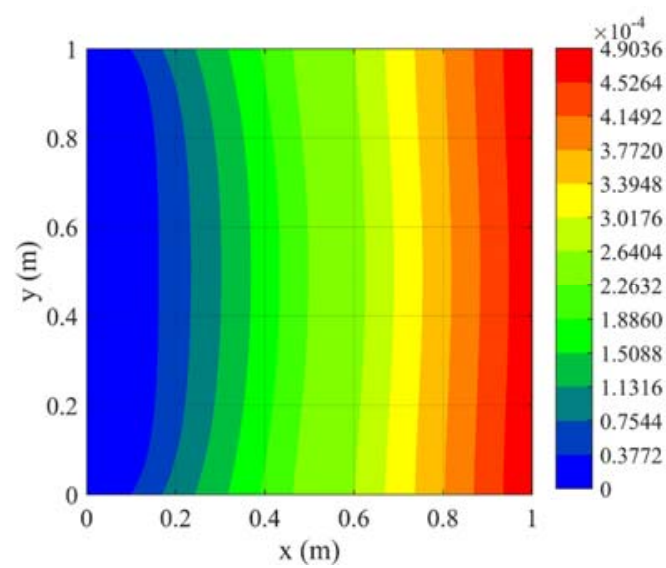

(b)

Fig. 12. Variation of displacement $u(m)$ of shell with $L / h=10$ (a) PD, (b) FEA results

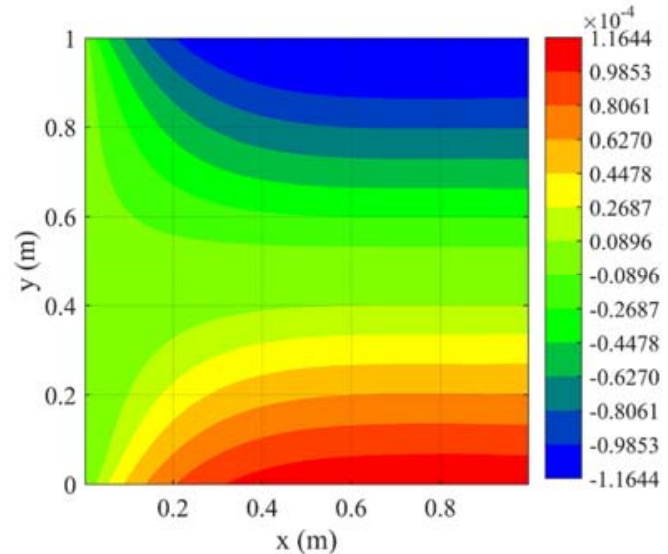

(a)

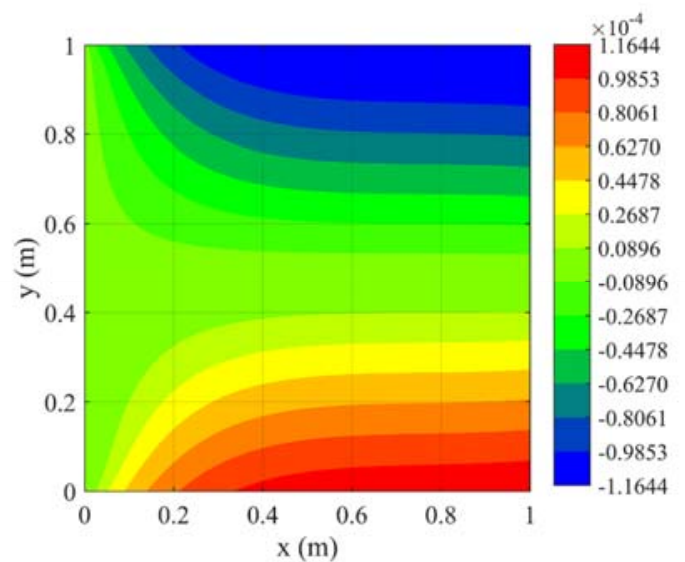

(b)

Fig. 13. Variation of displacement $v(\mathrm{~m})$ of shell with $L / h=10$ (a) PD, (b) FEA results

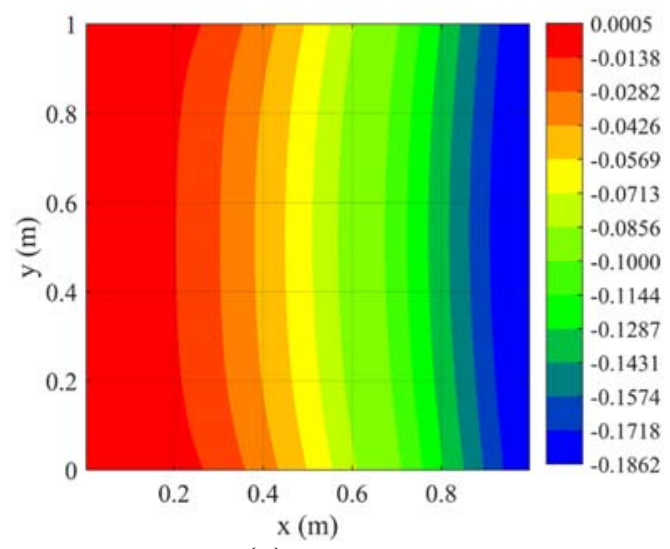

(a)

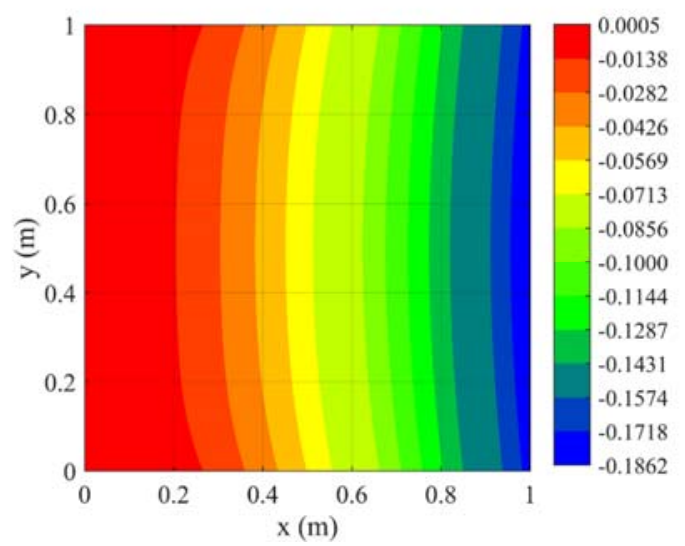

(b)

Fig. 14. Variation of displacement $w(\mathrm{~m})$ of shell with $L / h=10$ (a) PD, (b) FEA results 


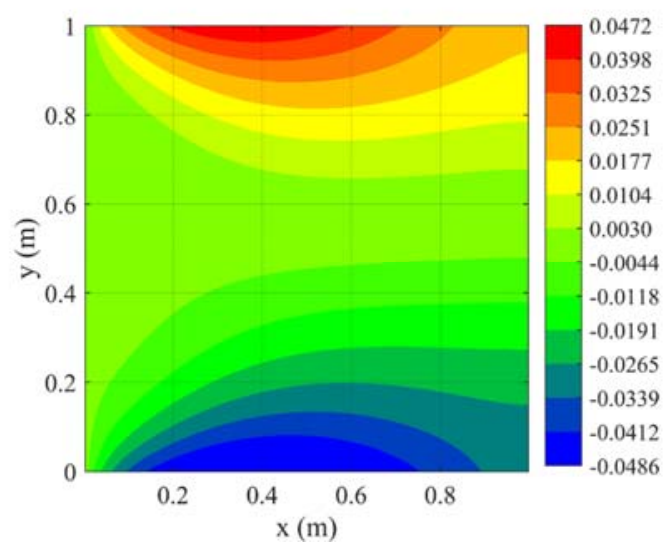

(a)

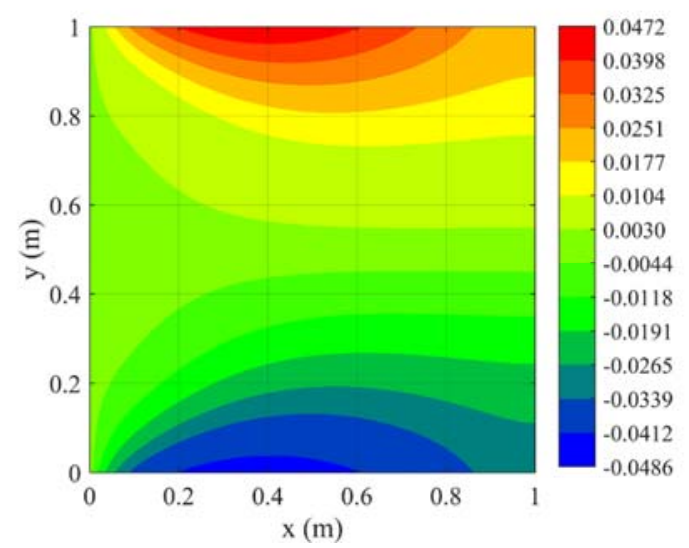

(b)

Fig. 15. Variation of rotation $\theta_{x}(\mathrm{rad})$ of shell with $L / h=10$ (a) PD, (b) FEA results

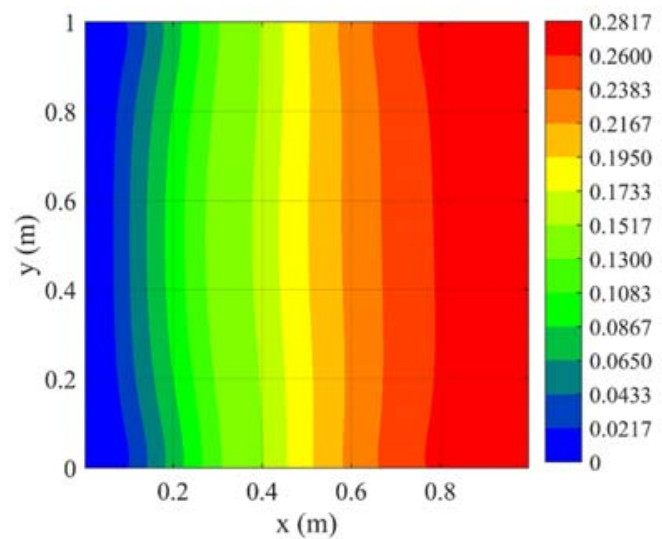

(a)

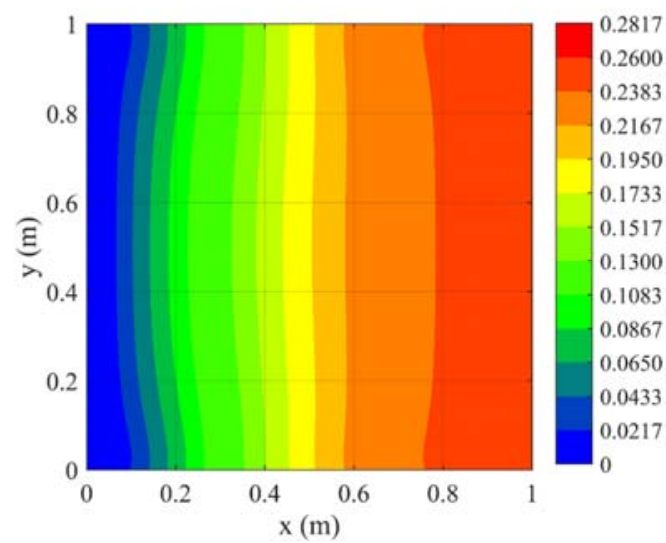

(b)

Fig. 16. Variation of rotation $\theta_{y}(\mathrm{rad})$ of shell with $L / h=10$ (a) PD, (b) FEA results

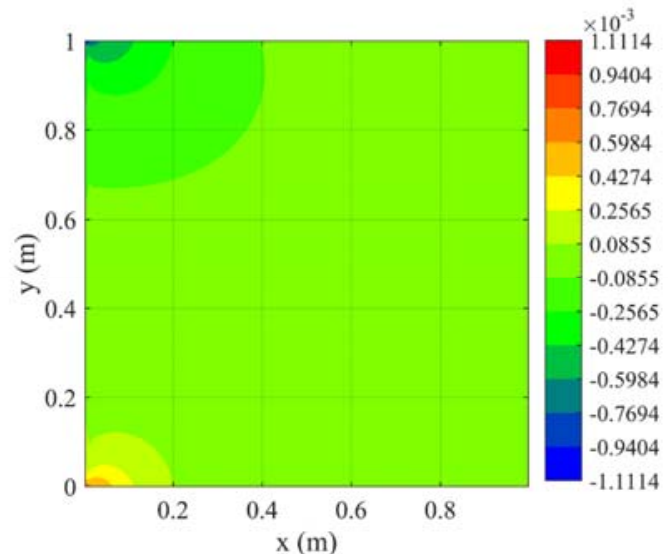

(a)

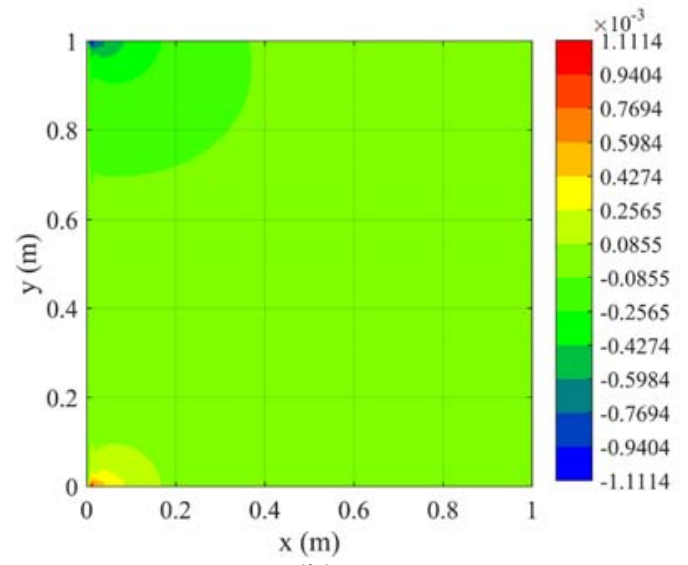

(b)

Fig. 17. Variation of rotation $\theta_{z}(\mathrm{rad})$ of shell with $L / h=10$ (a) PD, (b) FEA results 
In order to have a better comparison, the PD and FEA solution results along $y=W / 2$ and $x=L / 2$ are compared as shown in Fig. 18 and Fig. 19, respectively. As can be seen from the figures, PD and FEA solution results agree very well for both in-plane and out-of-plane deformations. Therefore, the developed PD model for thick flat shells is verified.
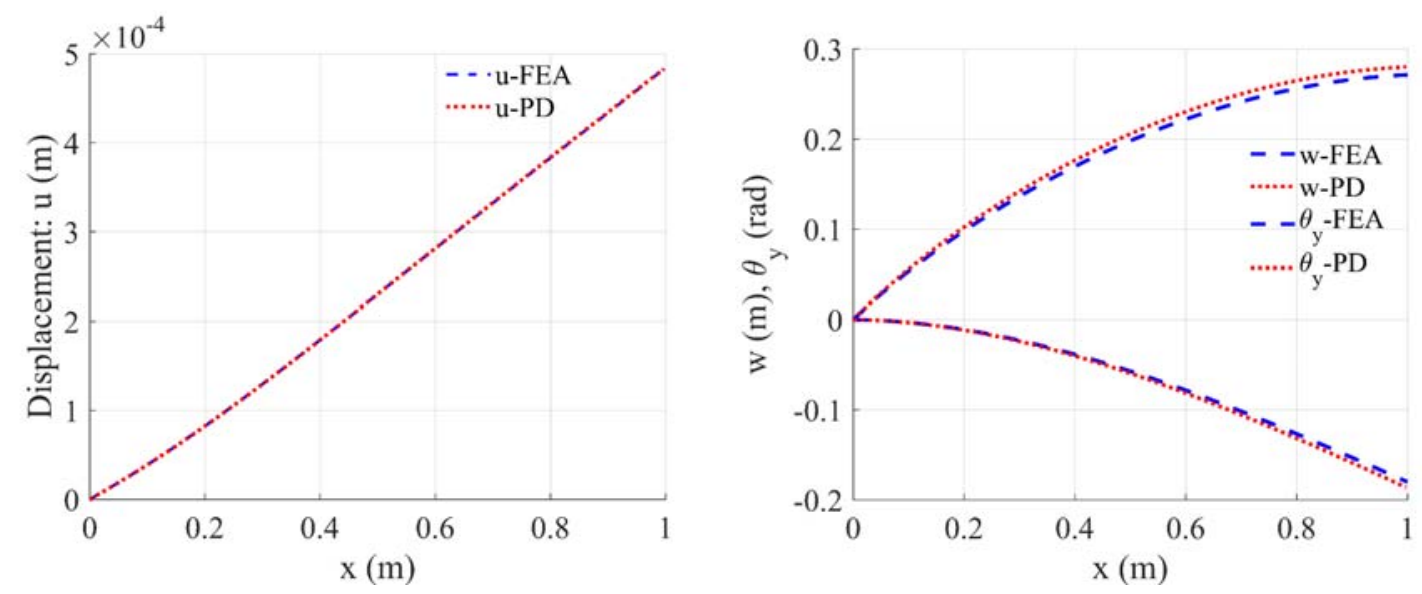

Fig. 18. Variation of DOFs $u(\mathrm{~m}), w(\mathrm{~m}), \theta_{y}(\mathrm{rad})$ along $y=W / 2$
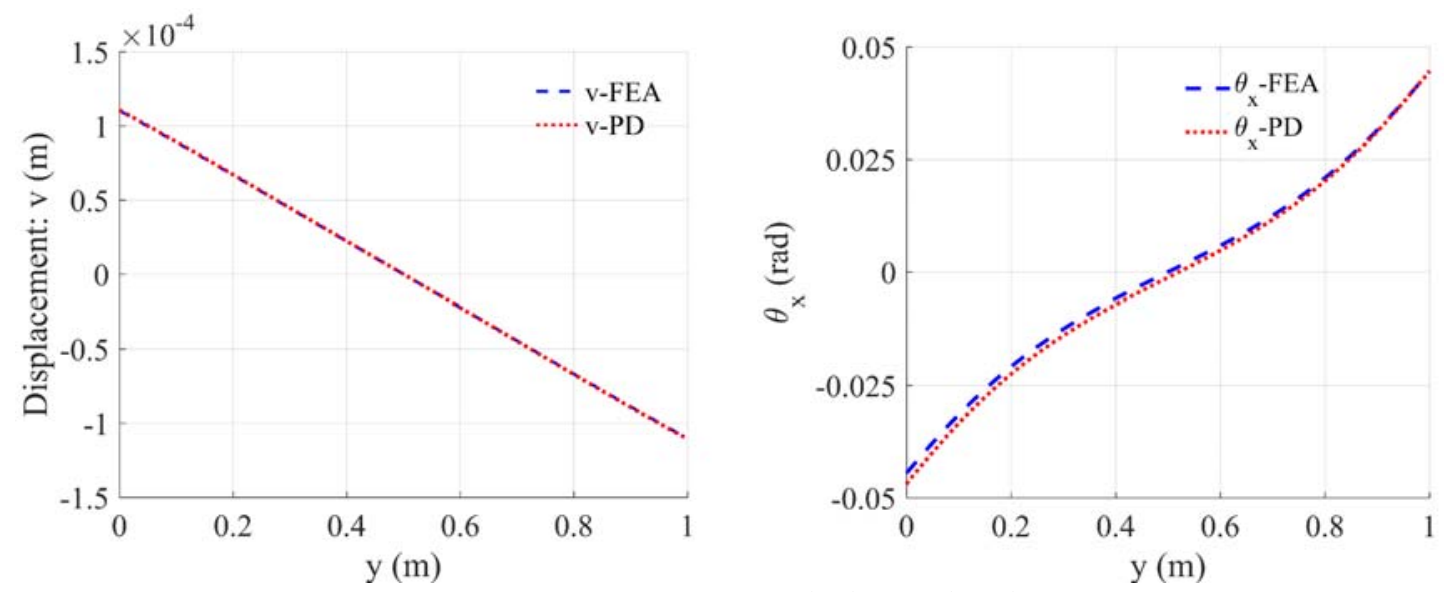

Fig. 19. Variation of DOFs $v(\mathrm{~m}), \theta_{x}(\mathrm{rad})$ along $x=L / 2$

In order to verify the developed PD model for thin shells, the flat shell is further investigated for three different cases corresponding to $L / h=10,100,1000$. In these cases, the shells are subjected to $p_{x}=1 \times 10^{2} \mathrm{~N} / \mathrm{m}$ and $p_{z}=-1 \times 10^{2} \mathrm{~N} / \mathrm{m}$. It is observed that, the relative errors between PD and FEA results for all DOFs are smaller than $3.71 \%$ for these three cases. Therefore, it can be concluded the developed PD model can be applicable for both thin and thick shell structures. In all cases, the mesh size is chosen as $150 \times 150$. Appendix F provides the PD and ANSYS solutions for thin shell with $L / h=100$. 
7.2. A curved shell subjected to constant static loading In order to verify the developed PD model for curved shells, a shell with a radius of $R$ is investigated as shown in Fig. 20(a). The shell is clamped at $\beta=75^{\circ}$ and it is subjected to a distributed load as $p_{z}=-1 \times 10^{7} \mathrm{~N} / \mathrm{m}$ at $\beta=90^{\circ}$. The shell has a thickness of $h=0.1 \mathrm{~m}$ and length of $L=0.7907 \mathrm{~m}$. It is made of steel with Young's modulus $E=2 \times 10^{11} \mathrm{~N} / \mathrm{m}^{2}$ and Poisson's ratio $v=0.27$.

The shell is discretized with uniform 150 integration points in each direction. In order to apply boundary conditions, fictitious layers of material points are added as shown in Fig. 20(b). All DOFs of these fictitious points are set equal to zero. In FEA model, same mesh size is used.

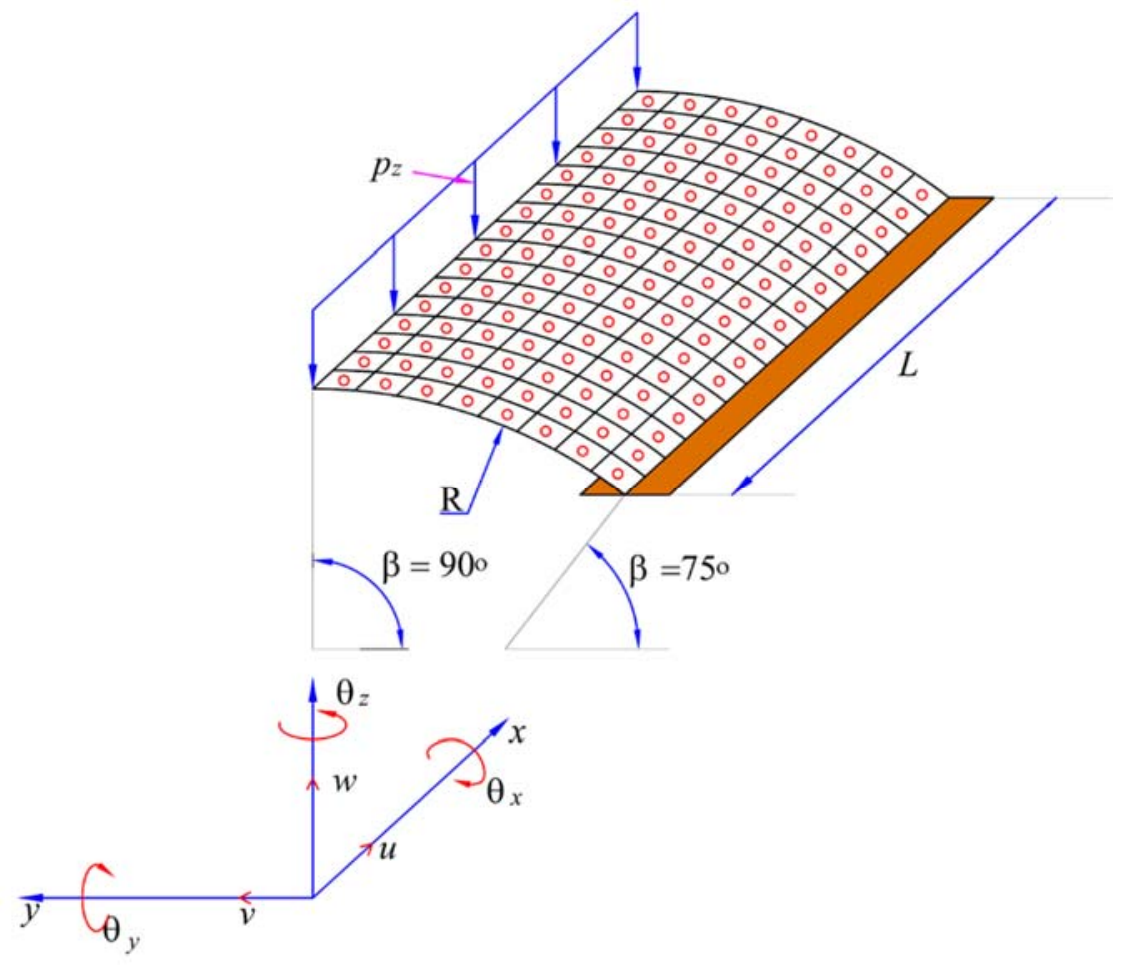

(a) 


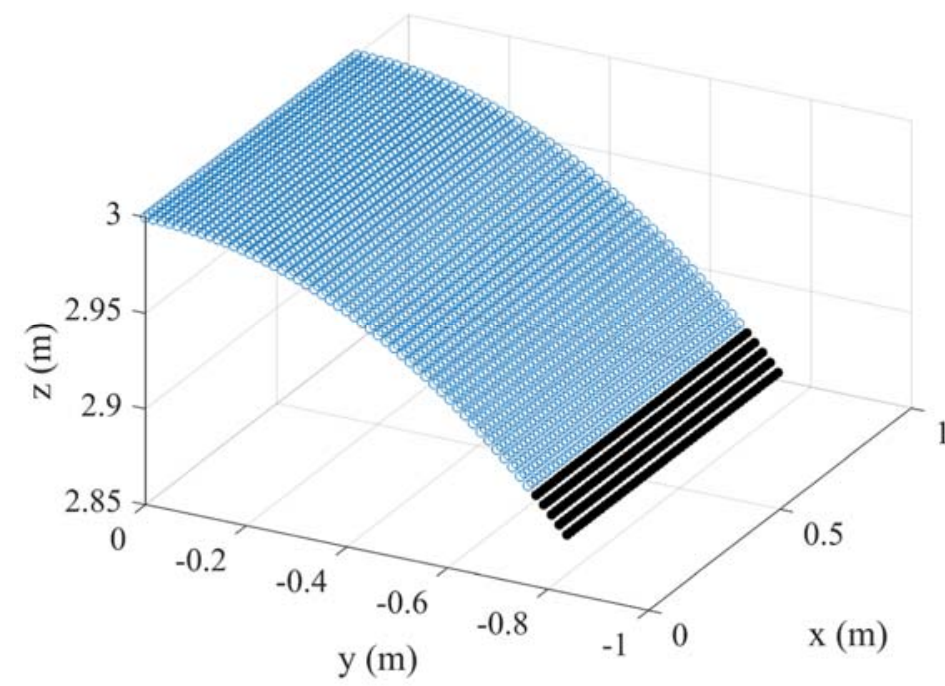

(b)

Fig. 20. A curved shell subjected to static loading (a) geometry, (b) model discretization

Similar to the previous example, the effects of $k_{0}$ to PD predictions with horizon size $\delta=3.015 \Delta x$ is investigated as shown in Fig. 21. In order to check the effect of the curvature, the curved shell is studied with two different values of radius, $R=1 \mathrm{~m}$ and $R=3 \mathrm{~m}$. As can be seen from the figure, the PD predictions for all degrees of freedom of the two curved shells have good agreement with the FEA solution when $k_{0} \geq 1$.Therefore, it can be concluded that the value of $k_{0}$ given in Eq. (33) can be chosen as $k_{0} \geq 1$ for both flat and curved shells. Therefore in the following sections $k_{0}=1$ is chosen.

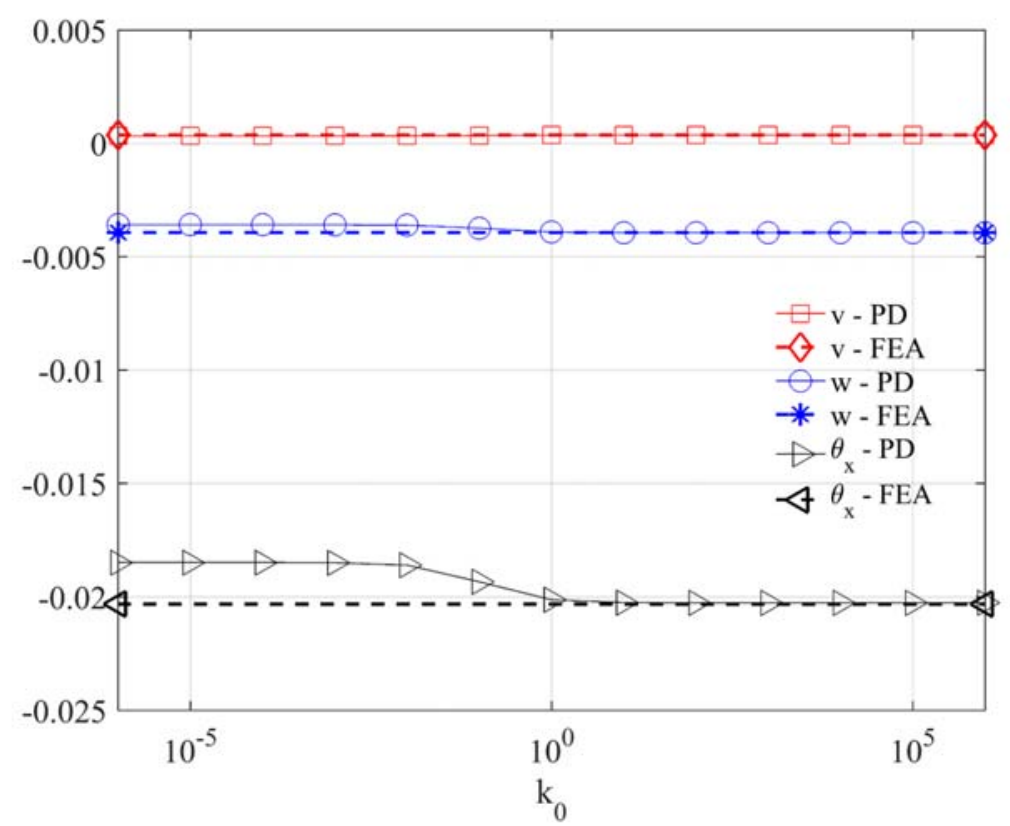

(a) 


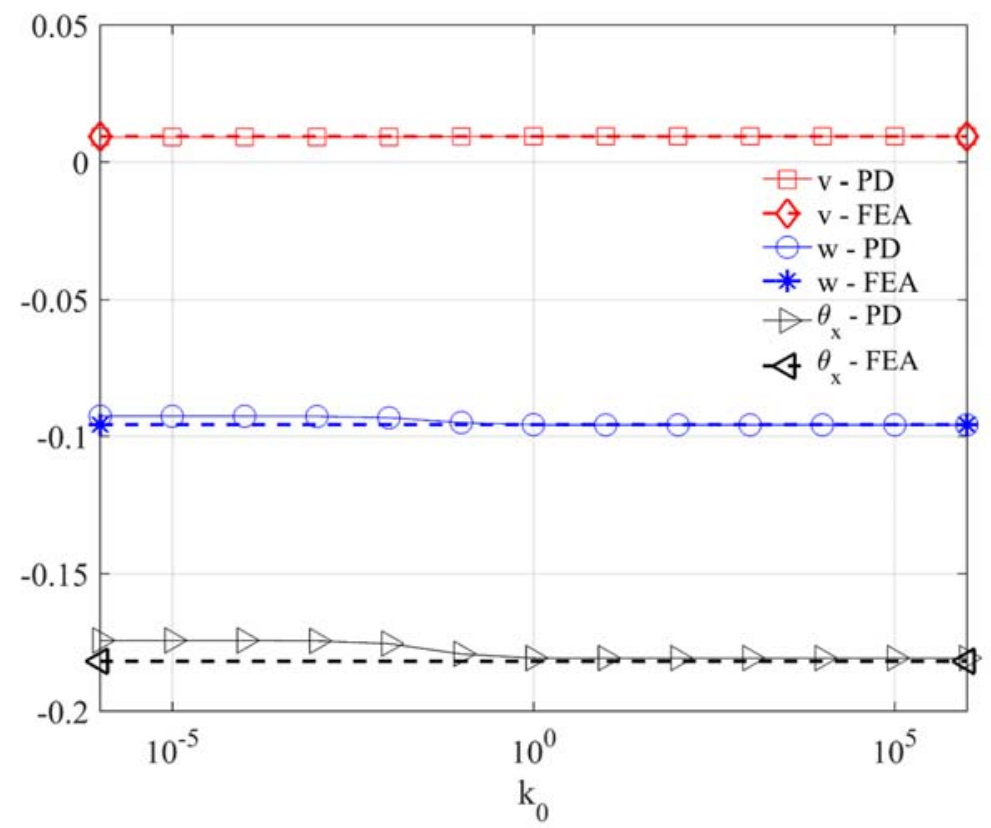

(b)

Fig. 21. Effect of torsional coefficient $k_{0}$ to solutions of curved shell radius of curvature
(a) $R=1 \mathrm{~m}$
(b) $R=3 \mathrm{~m}$ at $(x=L / 2, y=0, z=R)$

The effect of horizon size on PD results for curved shells are also investigated. Fig. 22 shows the relative errors between PD and FEA results for nonzero DOFs of material point located at $(x=L / 2, y=0, z=3)$ on the curved shell with $R=3 \mathrm{~m}$. As it can be seen from the figure, the PD results converge to FEA solution when $\delta=3.015 \Delta \mathrm{x}$ in which all the relative errors are smaller than $1.5 \%$. Therefore, $\delta=3.015 \Delta \mathrm{x}$ is chosen for the PD representation of curved shells. 


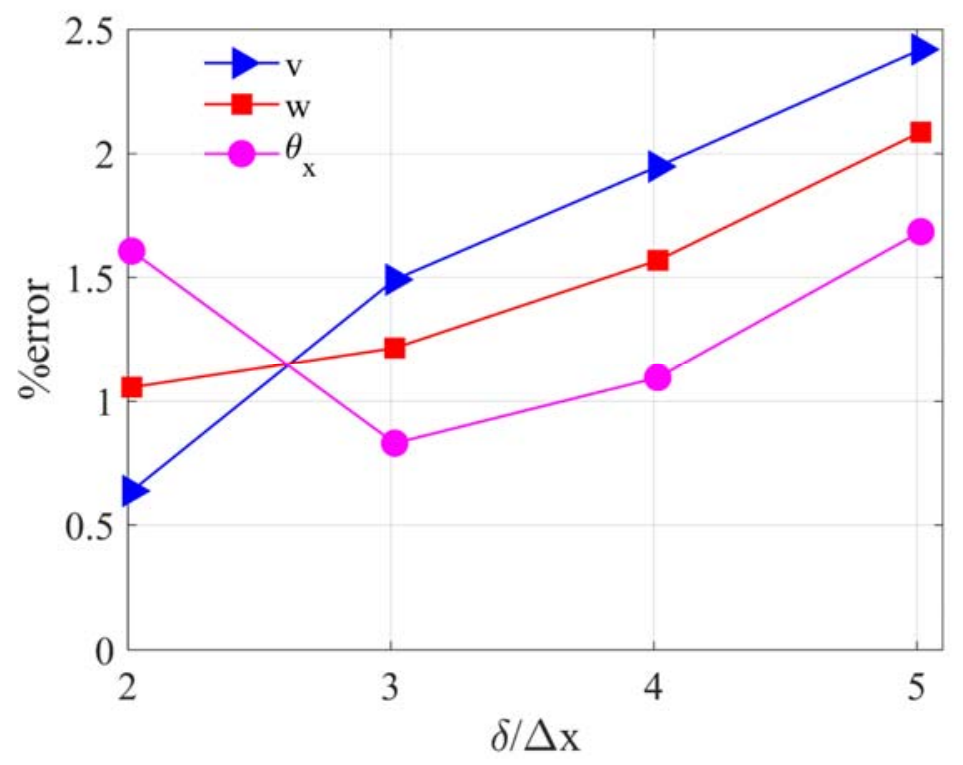

Fig. 22. Effect of horizon size on PD results at $(x=L / 2, y=0, z=3)$

After the stability of the PD prediction is investigated, the PD predictions with $k_{0}=1$ and $\delta=3.015 \Delta \mathrm{x}$ for the curved shell with radius $R=3 \mathrm{~m}$ are compared with FEA solution. Figs. 2328 show the variations of 6 DOFs of the shell in the deformed configuration. The results obtained from PD analysis agree very well with those in FEA. Moreover, as shown in Fig. 29, the deformed shape along the line of $x=L / 2$ captured in PD and FEA solutions are on top of each other which shows the accuracy of the developed PD model for curved shells. A good agreement between PD and FEA prediction results for thin curved shell with $L / h=100$ is also observed in Appendix F.2.

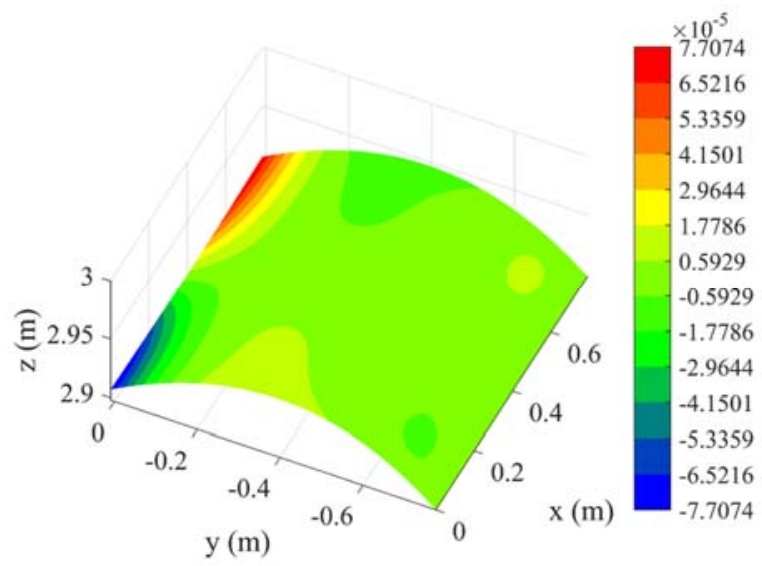

(a)

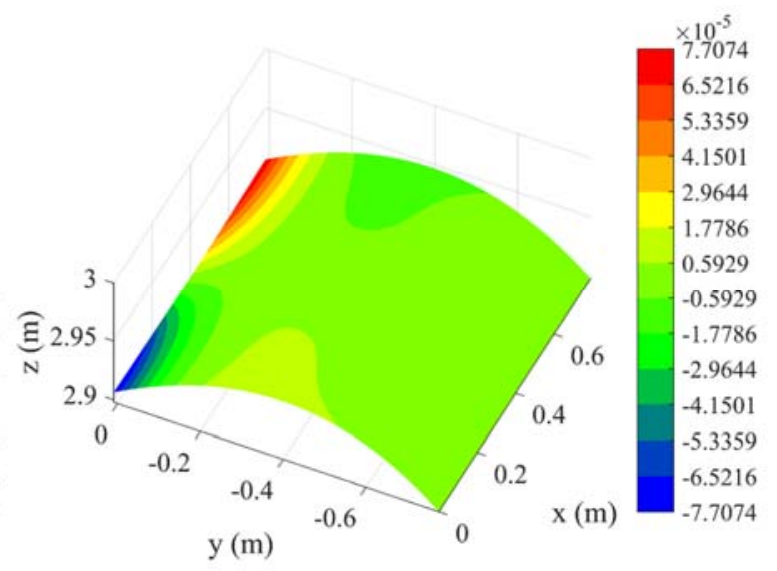

(b)

Fig. 23. Variation of displacement $u(\mathrm{~m})$ in deformed configuration (a) PD, (b) FEA results 


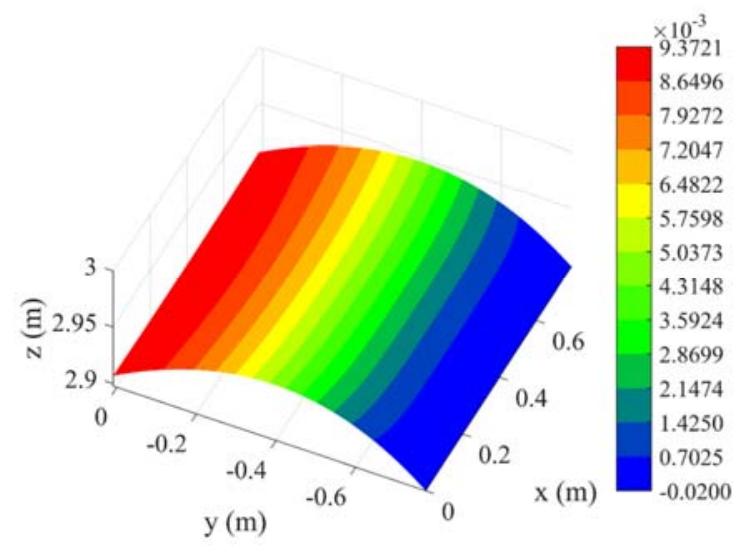

(a)

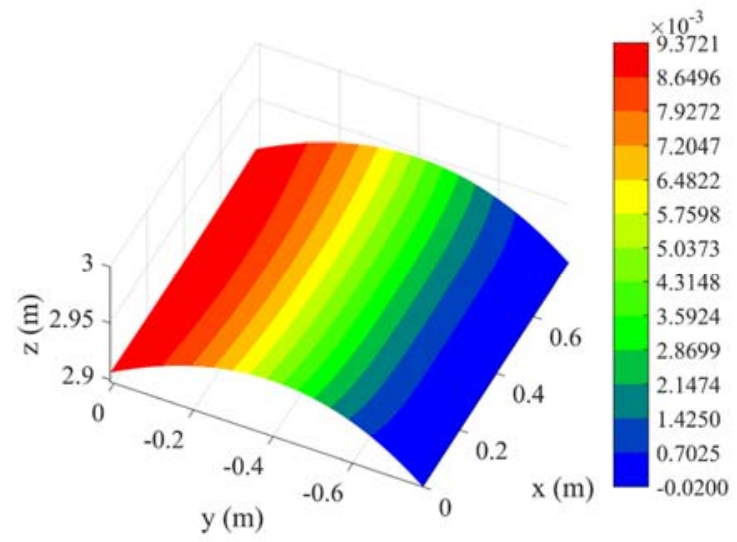

(b)

Fig. 24. Variation of displacement $v(\mathrm{~m})$ in deformed configuration (a) PD, (b) FEA results

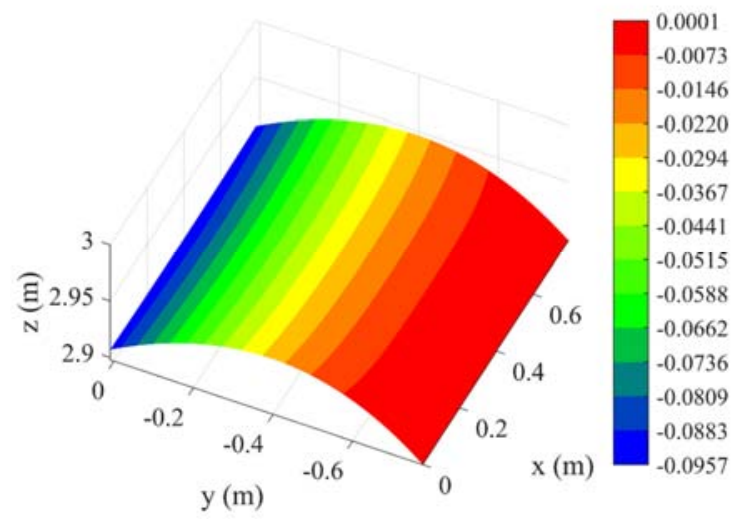

(a)

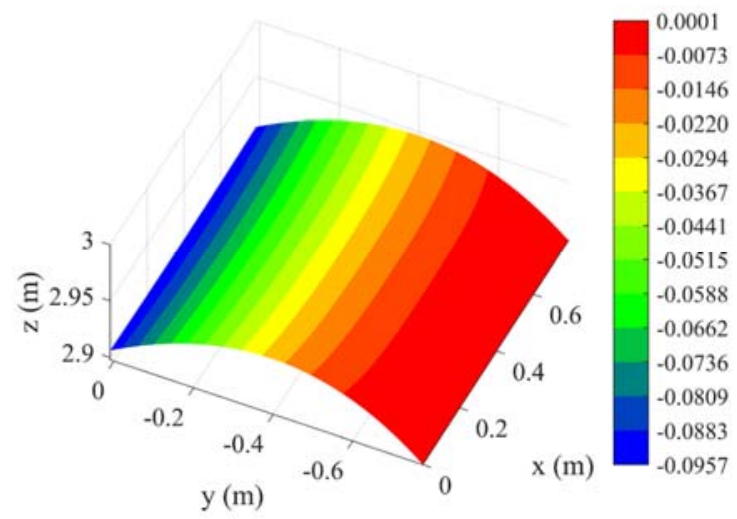

(b)

Fig. 25. Variation of displacement $w(\mathrm{~m})$ in deformed configuration (a) PD, (b) FEA results

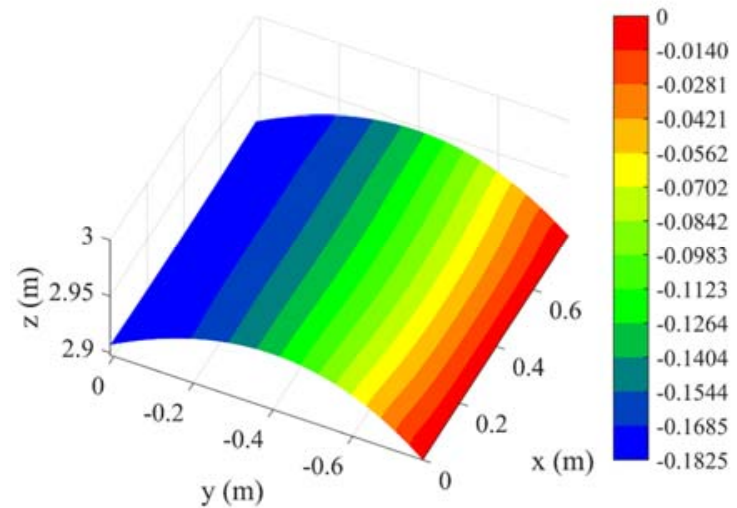

(a)

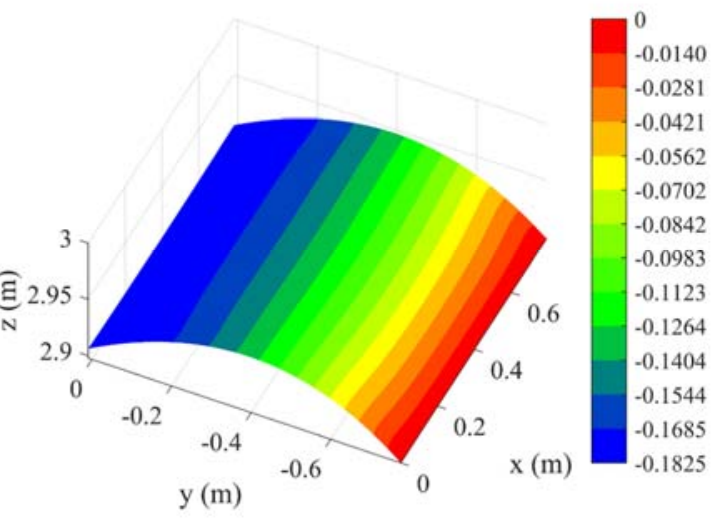

(b)

Fig. 26. Variation of rotation $\theta_{x}(\mathrm{rad})$ in deformed configuration (a) PD, (b) FEA results 


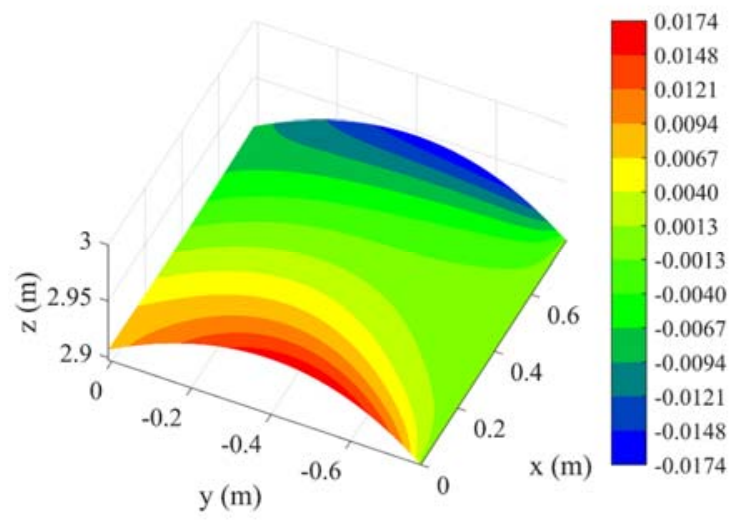

(a)

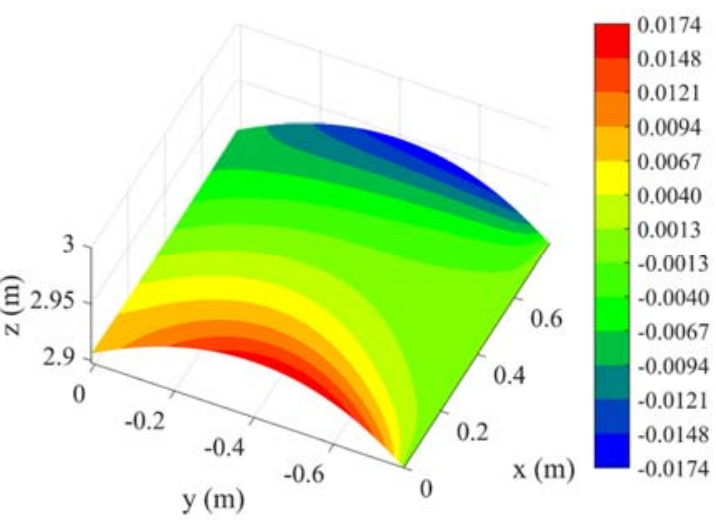

(b)

Fig. 27. Variation of rotation $\theta_{y}(\mathrm{rad})$ in deformed configuration (a) PD, (b) FEA results

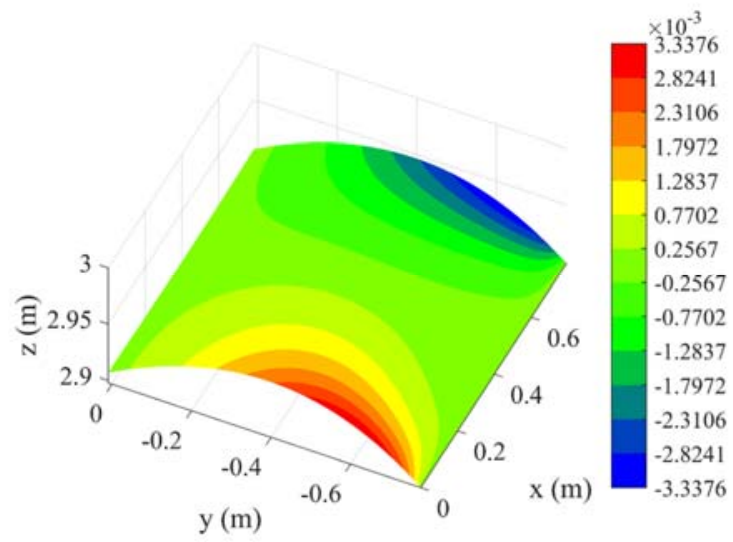

(a)

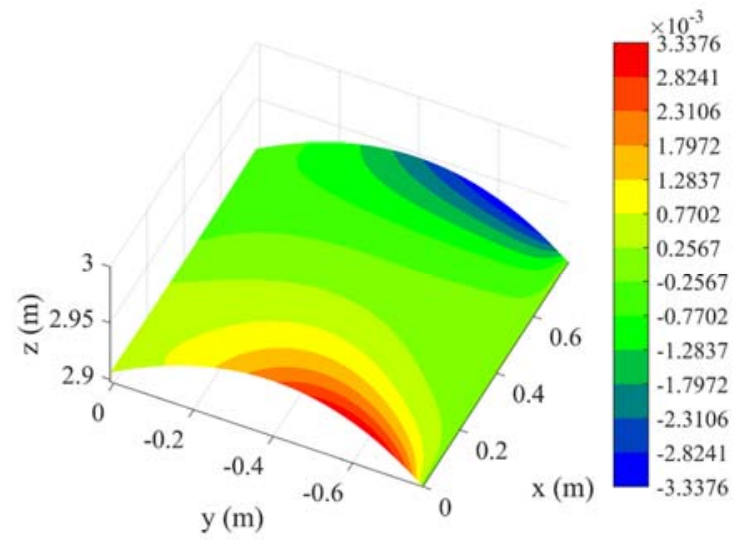

(b)

Fig. 28. Variation of rotation $\theta_{z}(\mathrm{rad})$ in deformed configuration (a) PD, (b) FEA results 


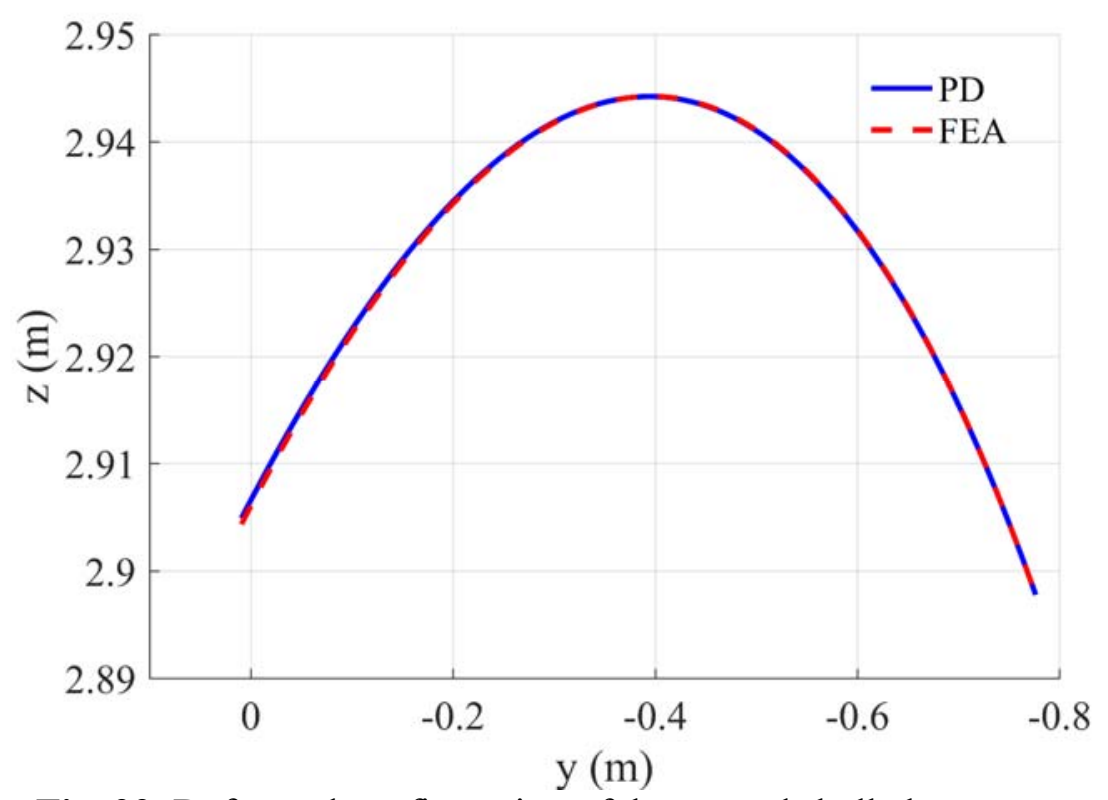

Fig. 29. Deformed configuration of the curved shell along $x=L / 2$

7.3. A stiffened structure subjected to constant static pressure

After verifying the developed PD model for flat and curved shells, this example is to verify the developed PD model for stiffened structures. As shown in Fig. 30(a), a shell structure made up from a cylindrical shell and four stringers is investigated. The cylindrical shell has a radius of $R=2 \mathrm{~m}$ and total length of $L=L_{1}+L_{2}+L_{3}=1.0472 \mathrm{~m}$ with $L_{1}=L_{3}=L / 4 \mathrm{~m}$. The side view of the stiffened structure is shown in Fig. 30(b). The height of each stringer is $H=0.1057 \mathrm{~m}$. All the shells have thickness of $h=0.1 \mathrm{~m}$ and they are made of steel with Young's modulus $E=2 \times 10^{11} \mathrm{~N} / \mathrm{m}^{2}$ and Poisson's ratio $v=0.27$. The cylindrical shell is subjected to constant pressure of $p_{0}=2 \times 10^{9} \mathrm{~N} / \mathrm{m}^{2}$. All four edges of the curved shell and two ends of each stringer, shown in black in Fig 30(a), are clamped.

In peridynamic model, the curved shell is discretized with $150 \times 150$ material points and each stringer is discretized with $150 \times 20$ material points. The horizon size is $\delta=3.015 \Delta x$. In order to apply boundary conditions, four fictitious layers of material points, shown in black, are added as shown in Fig. 31. All 6 DOFs of these fictitious material points are set equal to zero. In the FEA model, the same mesh size is used.

Figs. 32-37 show the variation of 6 DOFs of the shell structure. As shown in Fig. 34, due to the applied pressure, the middle region of the curved shell has the highest deformation in the vertical direction. As a result, due to the connection between the curved shell and the stringers, all stringers are twisted towards the centre of the cylindrical shell as shown in Fig. 32 and 33. As can be seen from the figures, the PD results agree very well with the FEA results which shows the accuracy of the developed PD model for stiffened structures. 


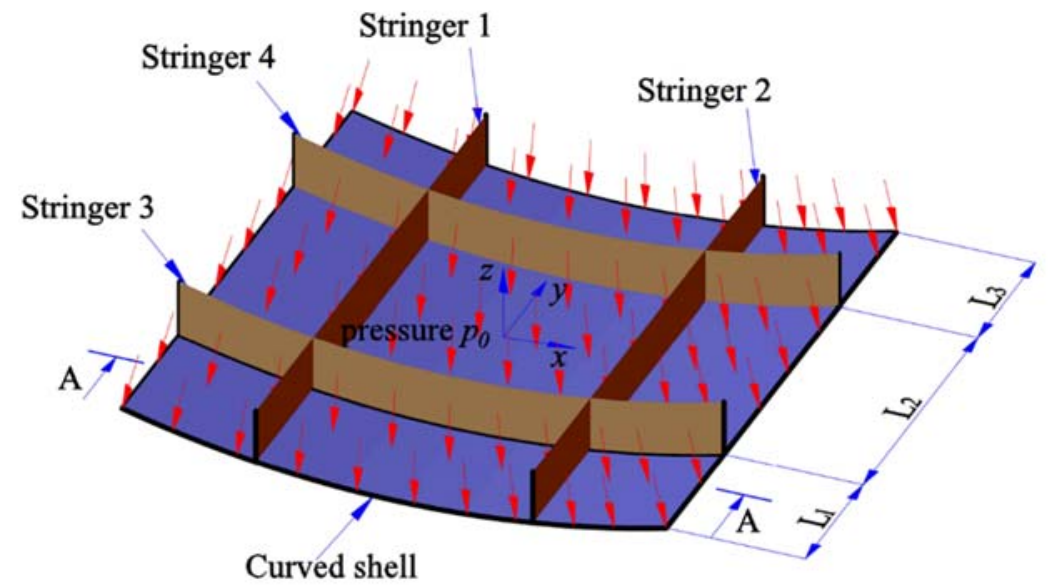

(a)

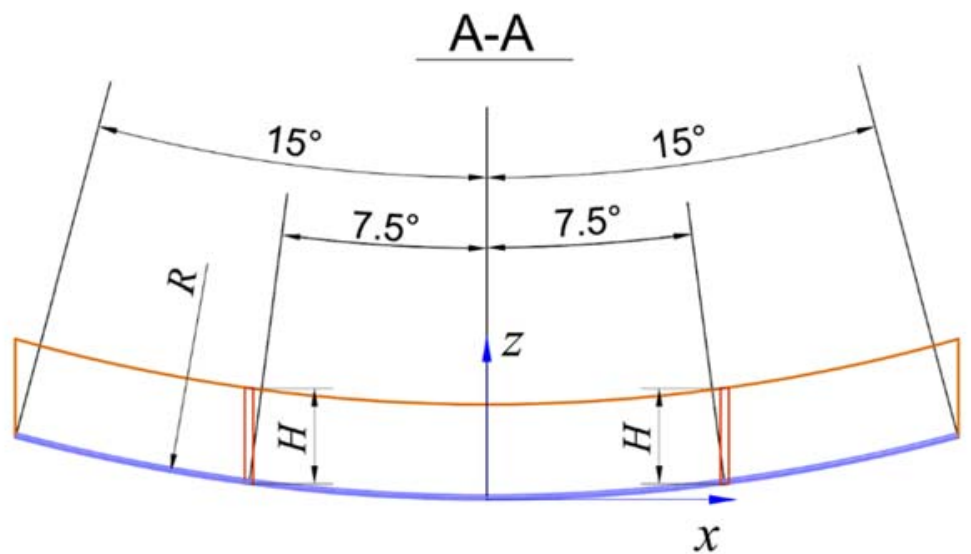

(b)

Fig. 30. Stiffened structure subjected to constant pressure (a) 3D view, (b) side view

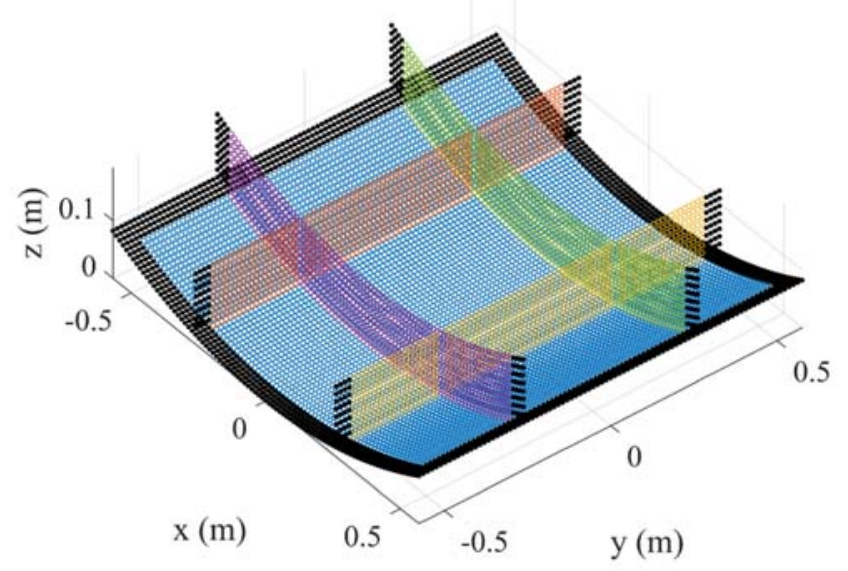

Fig. 31. Model discretization for stiffened structure 


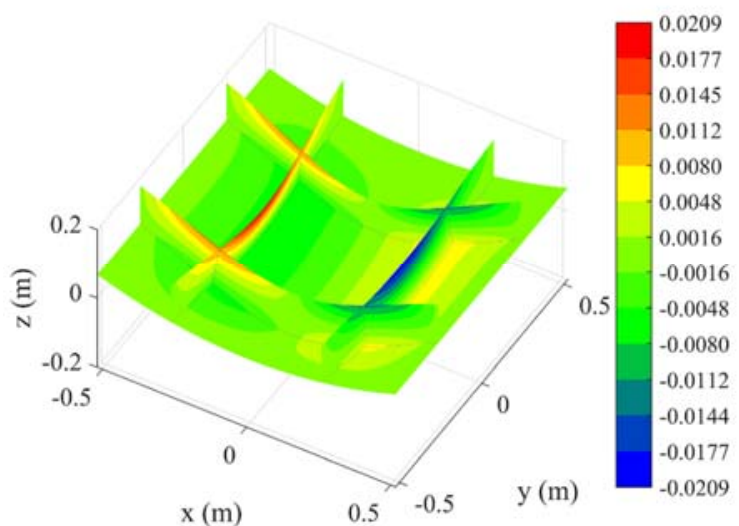

(a)

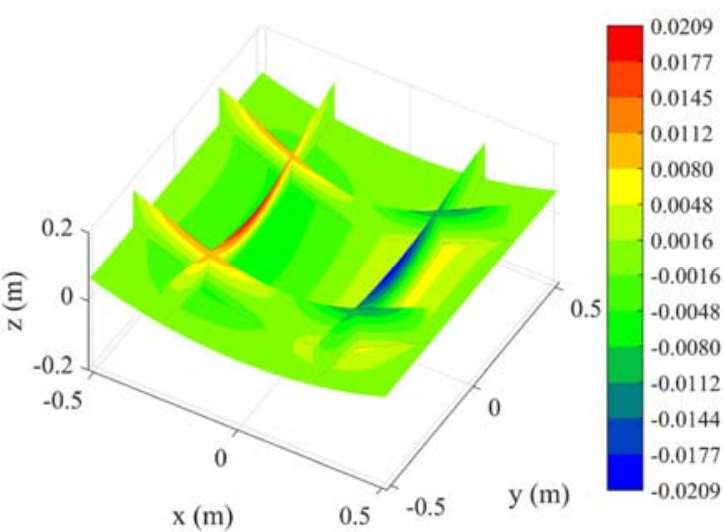

(b)

Fig. 32. Variation of displacement $u(\mathrm{~m})$ (a) PD, (b) FEA results

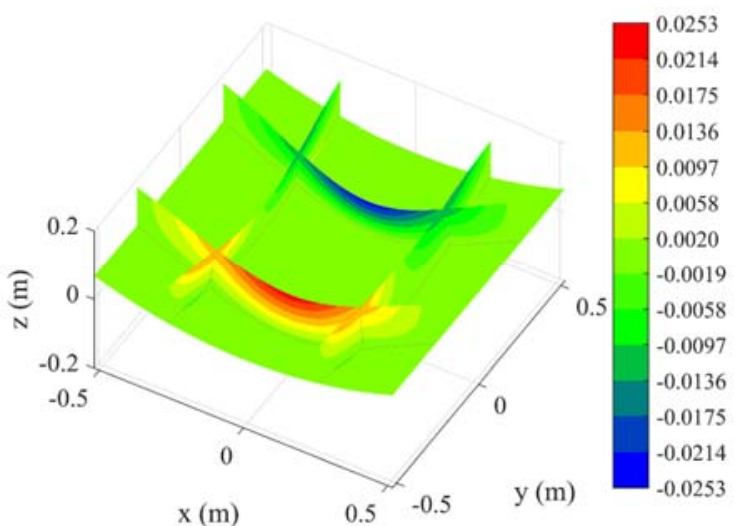

(a)

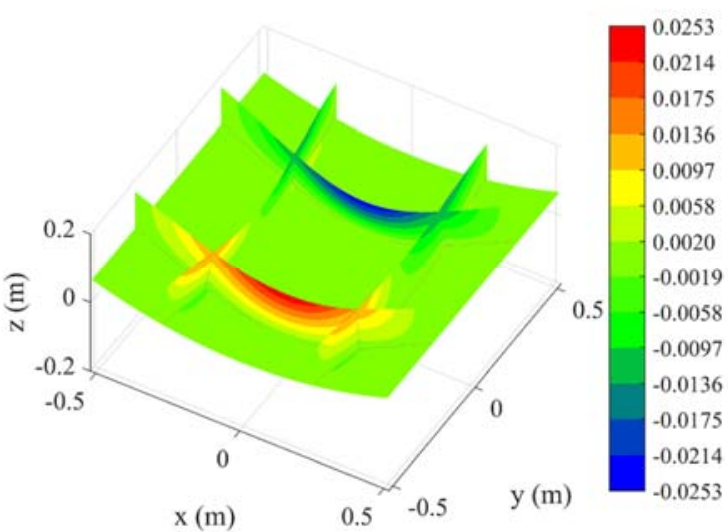

(b)

Fig. 33. Variation of displacement $v(\mathrm{~m})$ (a) PD, (b) FEA results

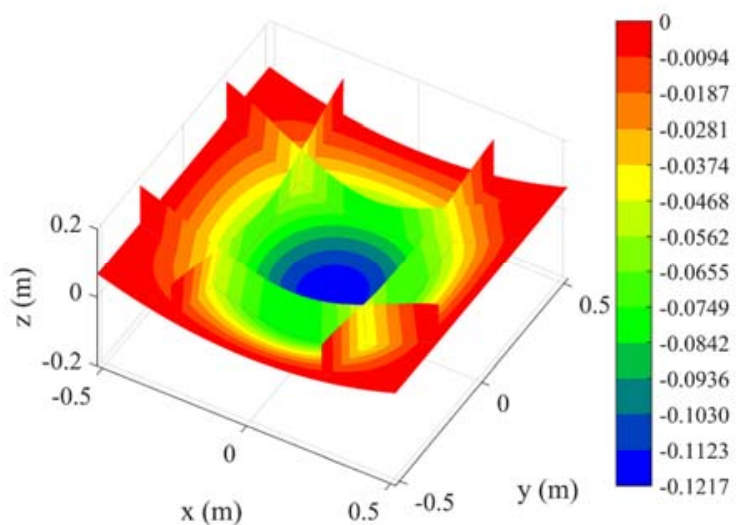

(a)

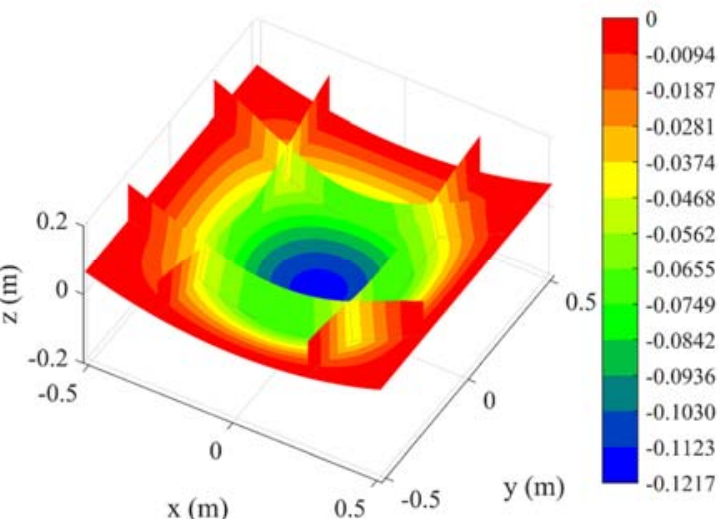

(b)

Fig. 34. Variation of displacement $w(\mathrm{~m})$ (a) PD, (b) FEA results 


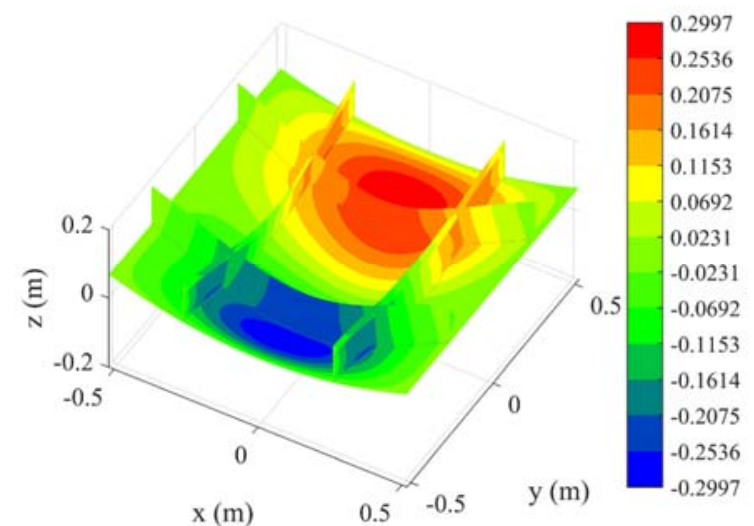

(a)

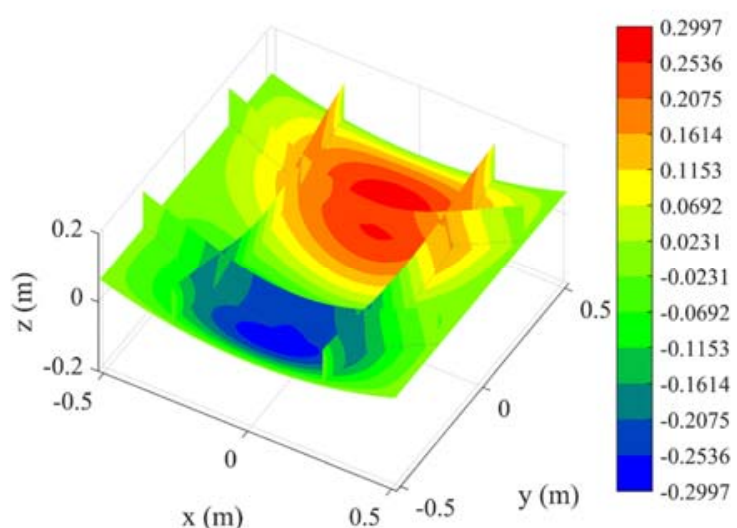

(b)

Fig. 35. Variation of rotation $\theta_{x}(\operatorname{rad})(a)$ PD, (b) FEA results

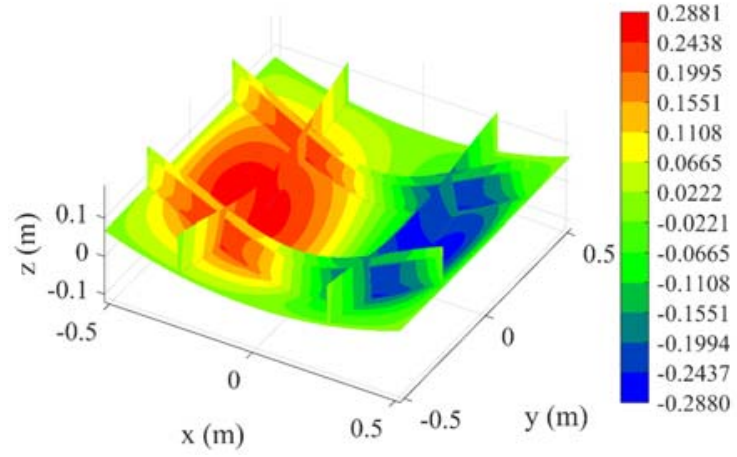

(a)

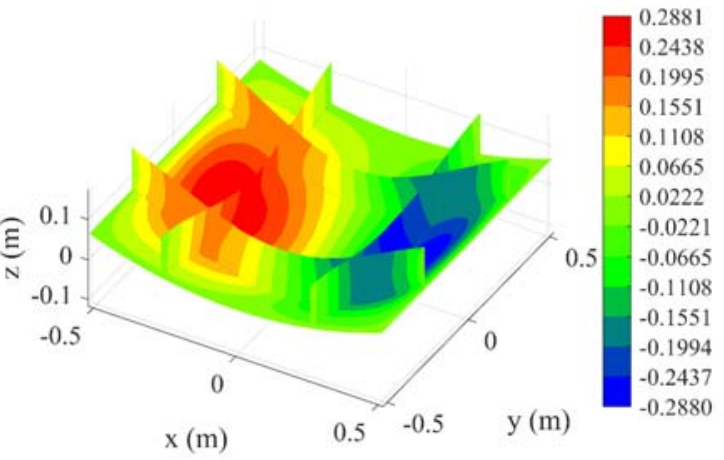

(b)

Fig. 36. Variation of rotation $\theta_{y}(\mathrm{rad})$ (a) PD, (b) FEA results

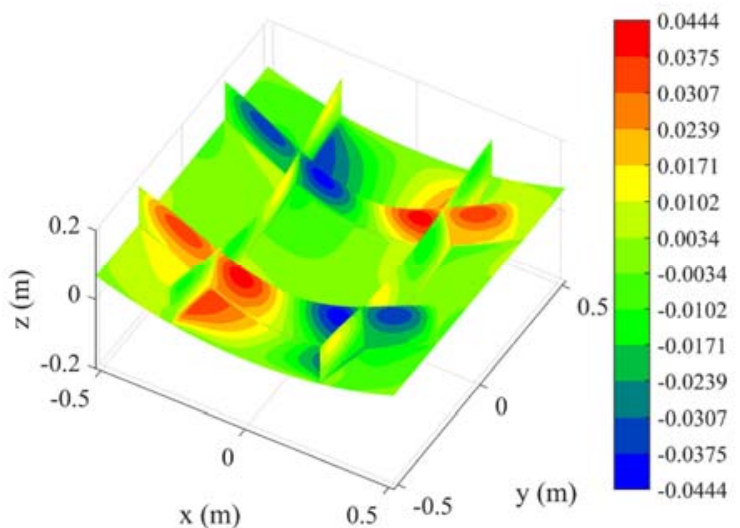

(a)

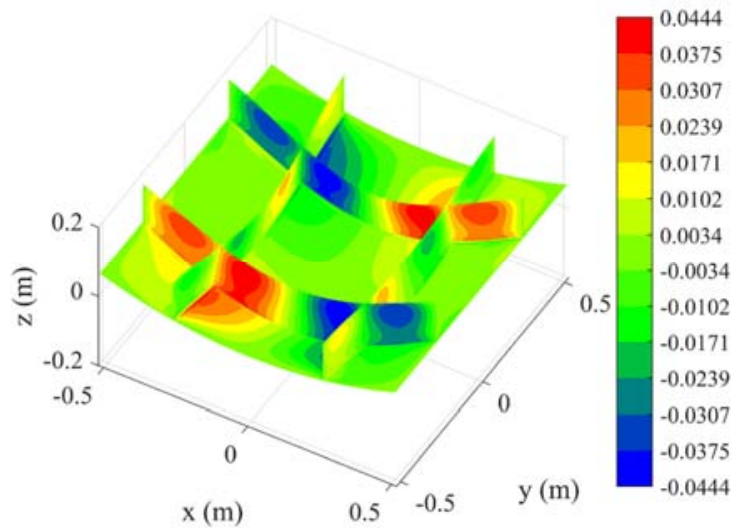

(b)

Fig. 37. Variation of rotation $\theta_{z}(\mathrm{rad})$ (a) PD, (b) FEA results 
7.4. Damage prediction for double torsion problem

After verifying the developed PD model for both mechanical and thermomechanical behaviour of shell structures, the damage process in a flat shell in double torsion problem is investigated. As shown in Fig. 38, the dimensions of the flat shell are $L=180 \mathrm{~mm}, W=60 \mathrm{~mm}, h=5 \mathrm{~mm}$ [39]. The shell has initial notch at $x=W / 2$ with initial notch length, $a=3.6 \mathrm{~mm}$. Along the line $x=W / 2$, an initial channel is created with $b=2.4 \mathrm{~mm}$ and $h_{n}=2.48 \mathrm{~mm}$ [39]. The material is marble with Young's modulus $E=26.68 \mathrm{GPa}$, Poisson's ratio $v=0.24[39]$, and fracture toughness $K_{c}=0.644 \mathrm{MPa} \sqrt{\mathrm{m}}[40]$. The critical energy release rate for this material is calculated as $G_{c}=K_{c}^{2} / E=15.545 \mathrm{~J} / \mathrm{m}^{2}$.

The shell is placed on 4 steel balls located at four corners with distances $\Delta=1.2 \mathrm{~mm}$ from corners. The shell is applied quasi-static loading on two points located at $x=W_{n}=23.35 \mathrm{~mm}$ and $x=W-W_{n}$ , respectively. The history of the total applied force, $F$ includes two stages as shown in Fig. 39 [39]. In the first stage, the load is increased to peak value of $F=158 \mathrm{~N}$ in $t=17.079 \mathrm{~s}$. Next, in the second stage, the load is slowly decreased to $F=120 \mathrm{~N}$ as shown in Fig 39.

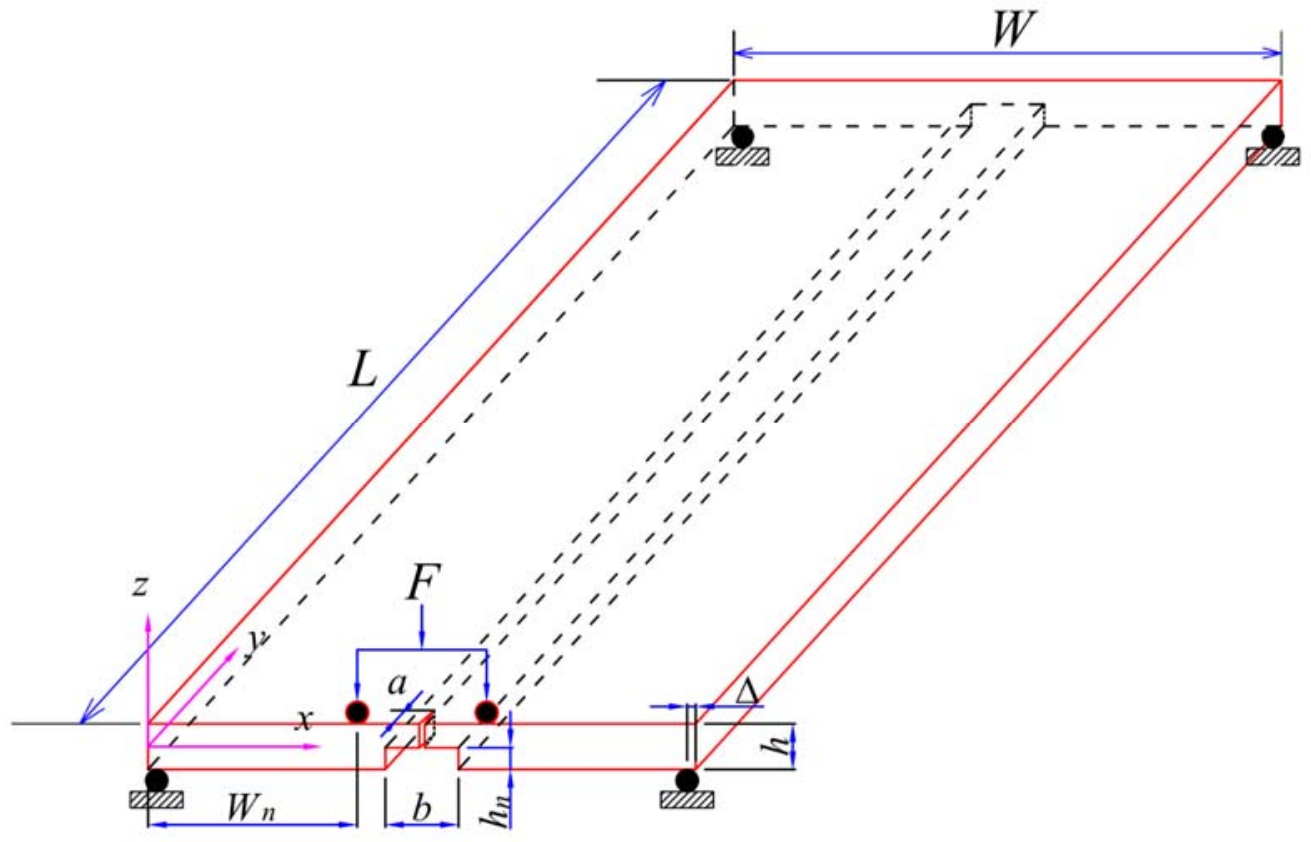

Fig. 38. Double torsion problem 


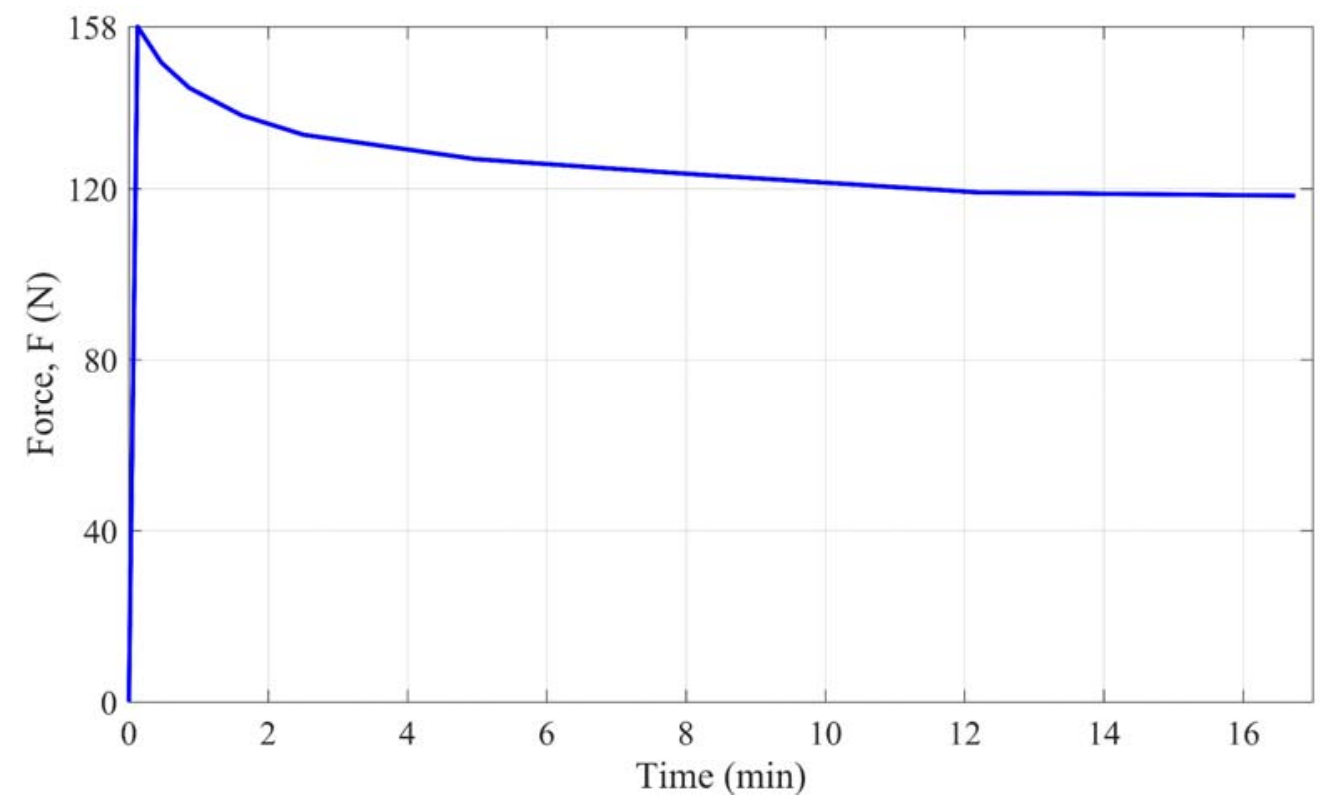

Fig. 39. Load-time curve [39]

In PD simulations, the model is discretized into $50 \times 150$ material points ( 50 layers of material points along $x$ direction and 150 layers along $y$ direction). In order to represent the initial trough, the thickness of material points located along the trough are defined as $h-h_{n}$, meanwhile other material points have a constant thickness $h$.

In order to apply loading conditions, two material points located at the locations of loading are defined. During the simulation, these two material points are equally applied force per unit area $\bar{b}_{z}=F / 2\left(\Delta x^{2}\right)$ in which the value of $F$ as function of time is given in Fig. 39. The adaptive dynamic relaxation methodology is used in the PD solution for this quasi-static problem. The problem is simulated in 40000 load steps, in which 15000 load steps are used for first loading stage, and 25000 load steps are used for second loading stage. Based on these information, the loading history in real time given in Fig. 39 is converted to loading history for quasi-static solution.

Fig. 40 shows the damage evolution on the flat shell predicted by using the developed PD model. The damage coefficient is plot in a range of $0 \leq \phi \leq 0.37$. Material points which have $\phi \geq 0.37$ are considered as completely damaged. As shown in Fig. 40(a), after $t=17.079 \mathrm{~s}$ when the load reaches to peak value, the structure is primarily damaged along the initial trough. The damage coefficients of material points along the trough are smaller than 0.1 which can be understood that the crack is not visibly propagated. At $t=22.794 \mathrm{~s}$, the crack starts propagating and the structure is more damaged along the channel as shown in Fig. 40(b). As shown in Figs. 40(c, d, e), the crack propagates along the channel up to the locations at, $y=50 \mathrm{~mm}, y=85 \mathrm{~mm}$ and $y=121 \mathrm{~mm}$ when $t=88.425 \mathrm{~s}, t=247.989 \mathrm{~s}$ and $t=567.116 \mathrm{~s}$, respectively. Fig. 40(f) shows the completely damaged experimental specimens in [39]. As it can be seen from the figures, the crack paths captured by PD simulation and experiment [39] agree very well. 
Fig. 41 shows the variation of crack length and crack growth velocity captured by PD simulation and experiments in [39]. As can be seen from the figure, both the crack length and crack growth velocity captured by PD simulation agree with the experiment which show the capacity of the developed PD model.

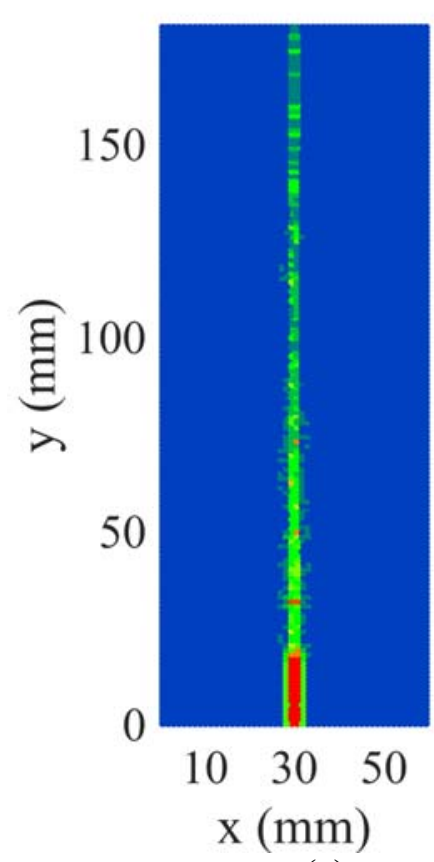

(a)

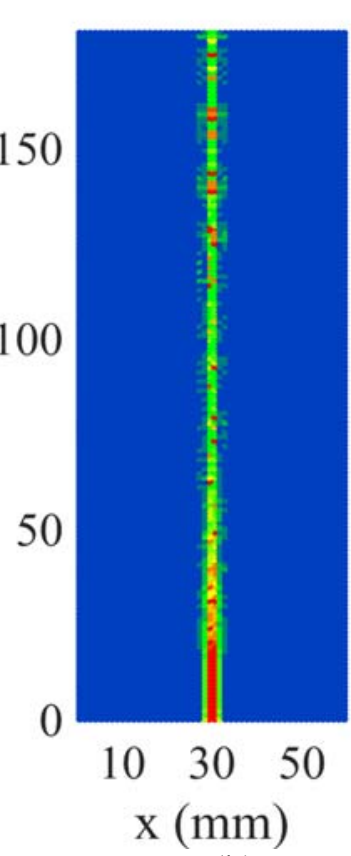

(b)

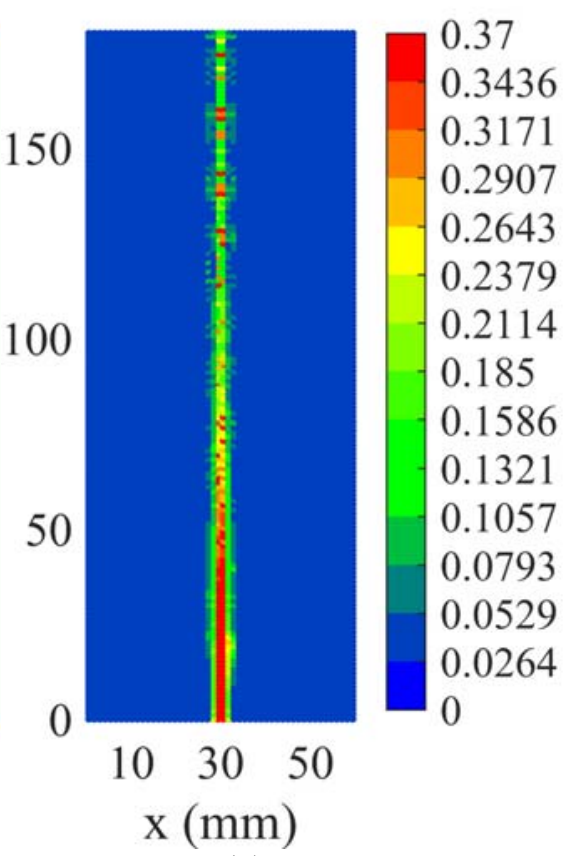

(c)

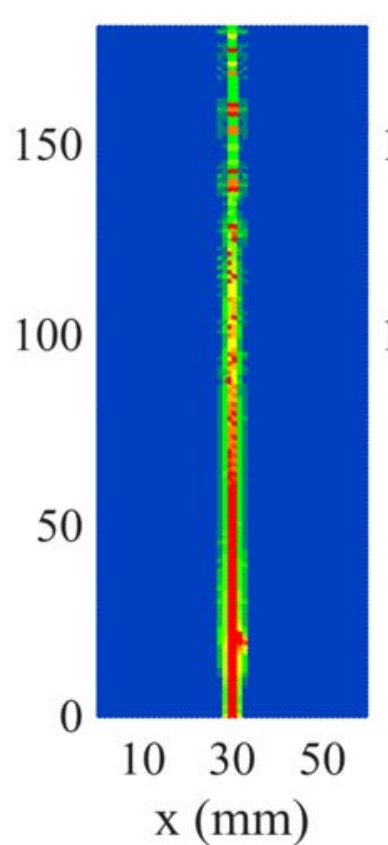

(d)

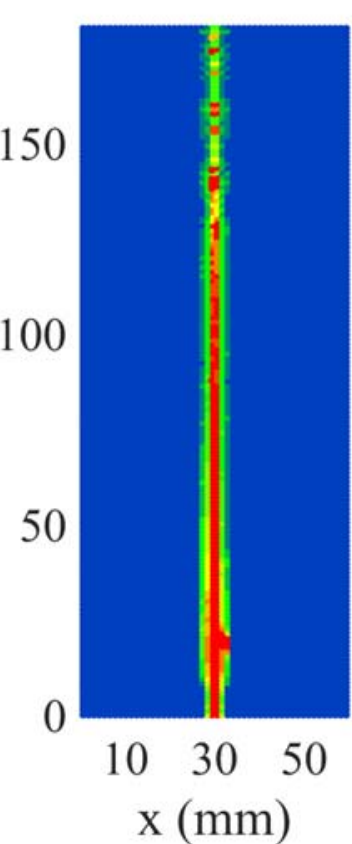

(e)

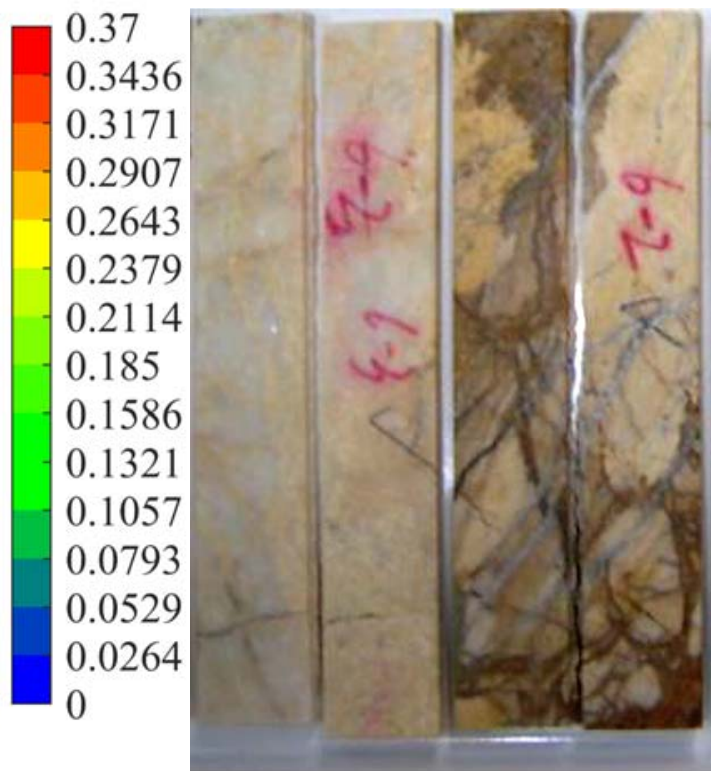

(f)

Fig. 40. Variation of damage coefficient, $\phi$ at (a) $t=17.079 \mathrm{~s}$, (b) $t=22.794 \mathrm{~s}$, (c) $t=88.425 \mathrm{~s}$, (d) $t=247.989 \mathrm{~s}$, (e) $t=567.116 \mathrm{~s}$, (f) completely damaged specimens [39] 


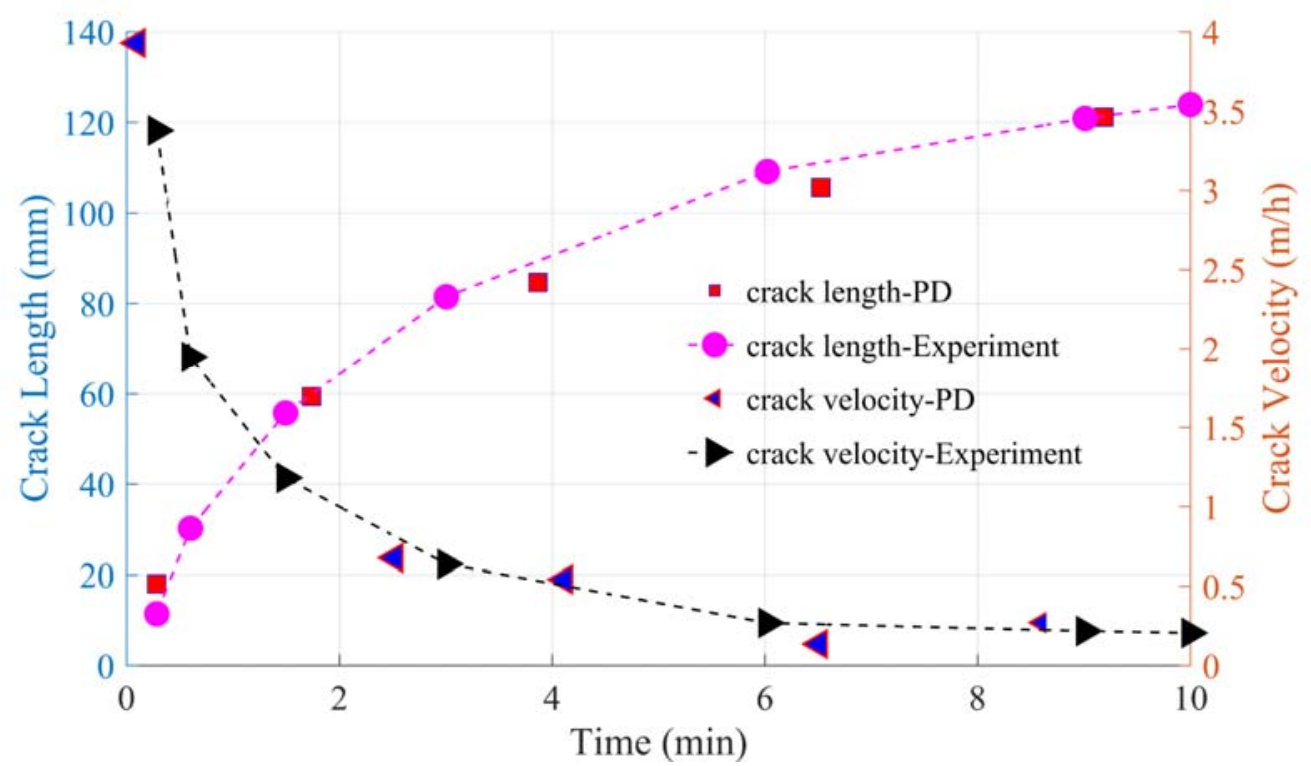

Fig. 41. Variation of crack length and crack growth velocity (experiment [39])

7.5. A flat shell with a pre-existing crack

In this section, a flat shell with dimensions of $L=0.8 \mathrm{~m}, W=0.6 \mathrm{~m}$, and thickness of $h=0.01 \mathrm{~m}$ is investigated as shown in Fig. 42. The flat shell has a pre-existing crack at the middle with the crack length of $2 a=W / 10$. The orientation of the initial crack is defined by angle $\beta$ as shown in Fig. 42. Two different cases of initial crack orientations: $\beta=90^{\circ}$ (case 1) and $\beta=45^{\circ}$ (case 2) are investigated.

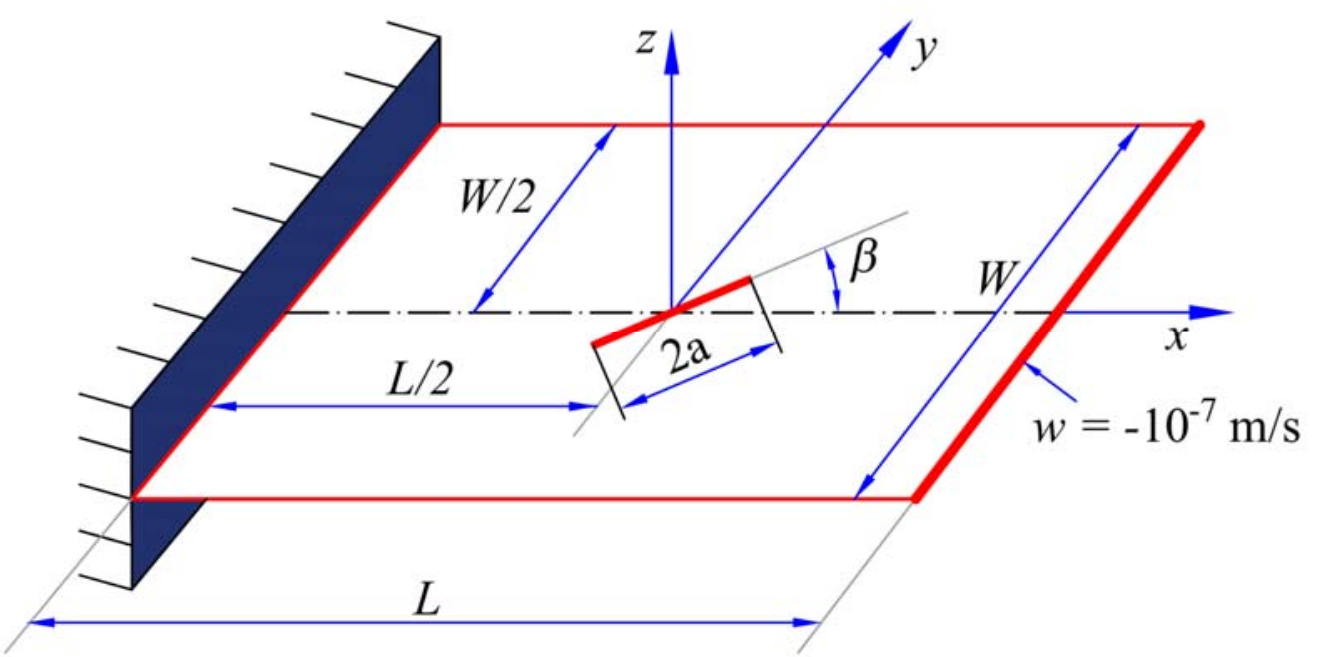

Fig. 42. A flat shell with pre-existing crack 
The flat shell is made of steel with Young's modulus $E=2 \times 10^{11} \mathrm{~N} / \mathrm{m}^{2}$ and Poisson's ratio $v=0.27$. The fracture toughness of steel is $K_{I C}=70 \times 10^{6} \mathrm{Nm}^{-3 / 2}$ [41] and the critical energy release rate is $G_{c}=2.2714 \times 10^{4} \mathrm{~J} / \mathrm{m}^{2}$.

The flat shell is fixed on the left edge and it is applied incremental vertical displacement is applied by increasing the displacement by $\Delta w=-10^{-7} \mathrm{~m}$ on the right edge as shown in Fig. 42. In PD model, the shell is discretized with a mesh size of $\Delta x=0.008 \mathrm{~m}$. Similar to Section 7.1 , three fictitious layers of material points are added on the left and all degrees of freedom of these fictitious point and material points located at $x=-L / 2$ are set equal to zero.

Fig. 43 presents the damage evolution on the flat shell with initial crack orientation $\beta=90^{\circ}$ (case 1). Fig. 43(a) presents the initial damage on the flat shell. As the applied displacement increases, the crack propagates continuously and by remaining the initial orientation as shown in Figs. 43(bd). As it can be seen from the figures, the crack propagates symmetrically in both positive and negative $y$ directions. As shown in Fig. 43(d), when the applied displacement $w=-0.0366 \mathrm{~m}$, the crack propagates and nearly reaches the boundary edges of the flat shell.

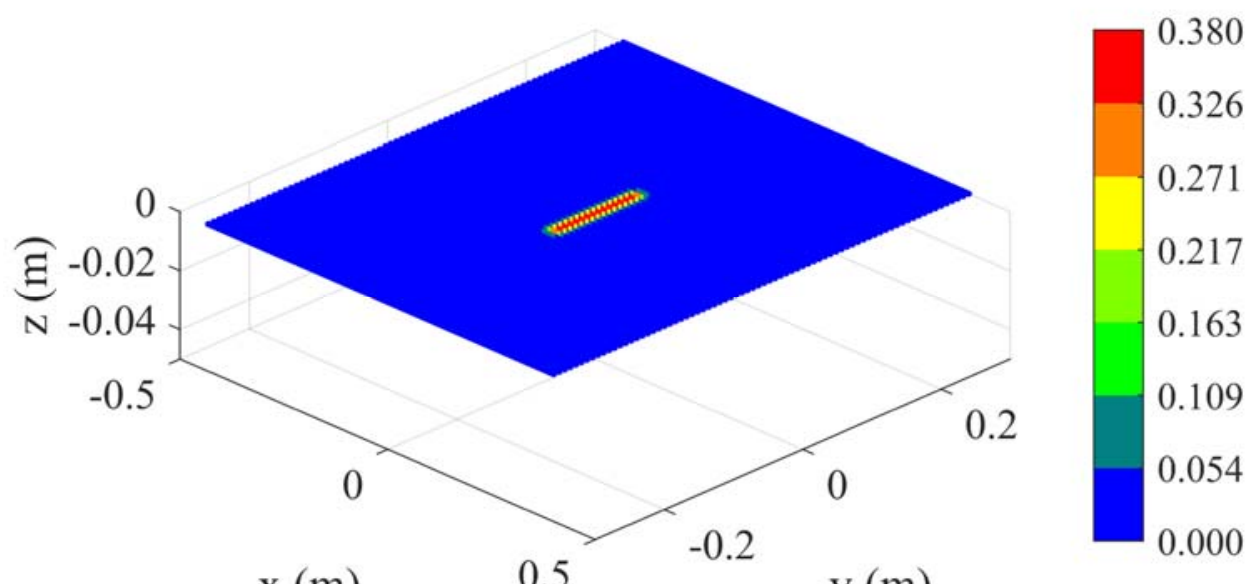

(a)

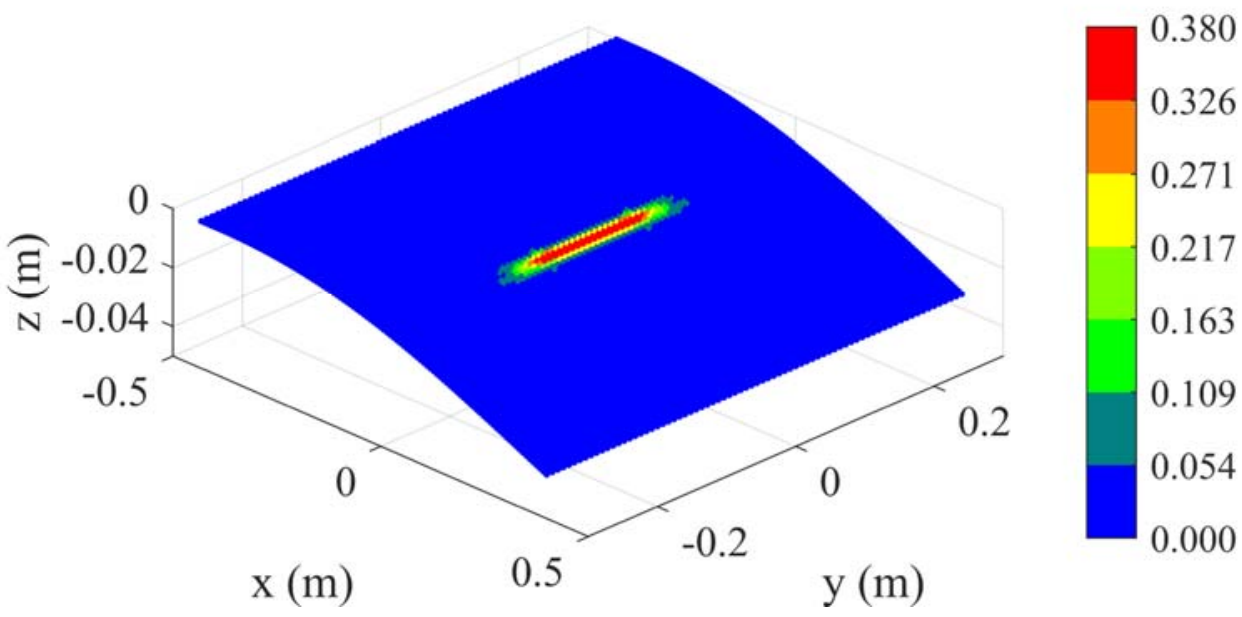


(b)

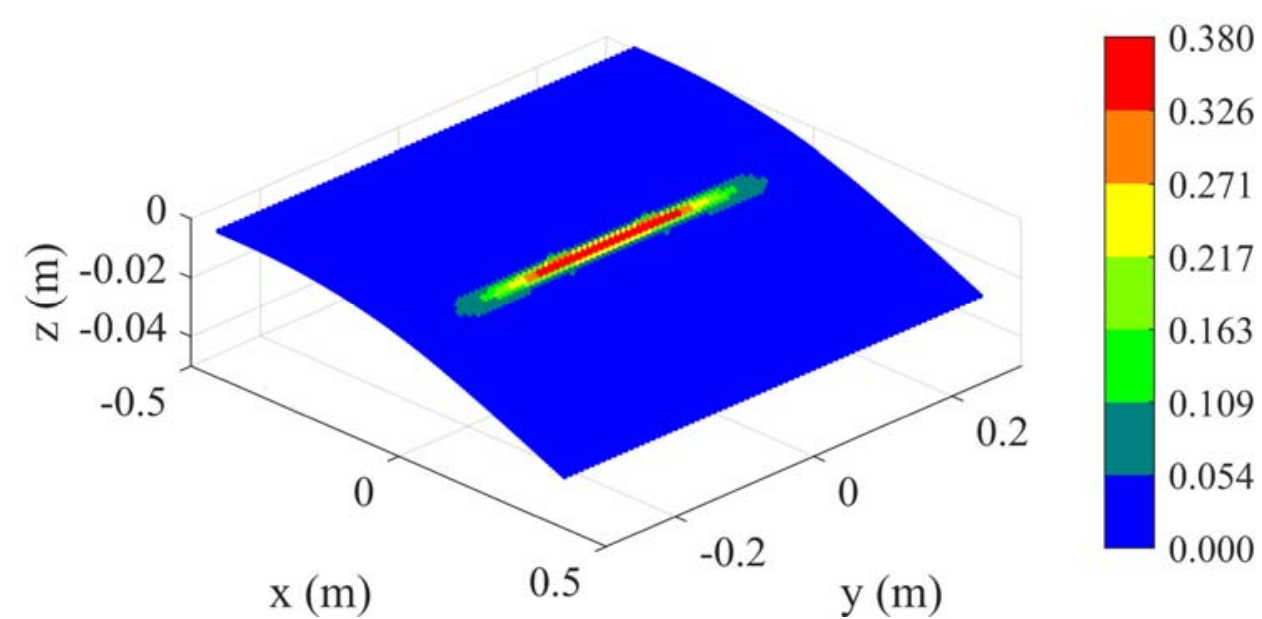

(c)

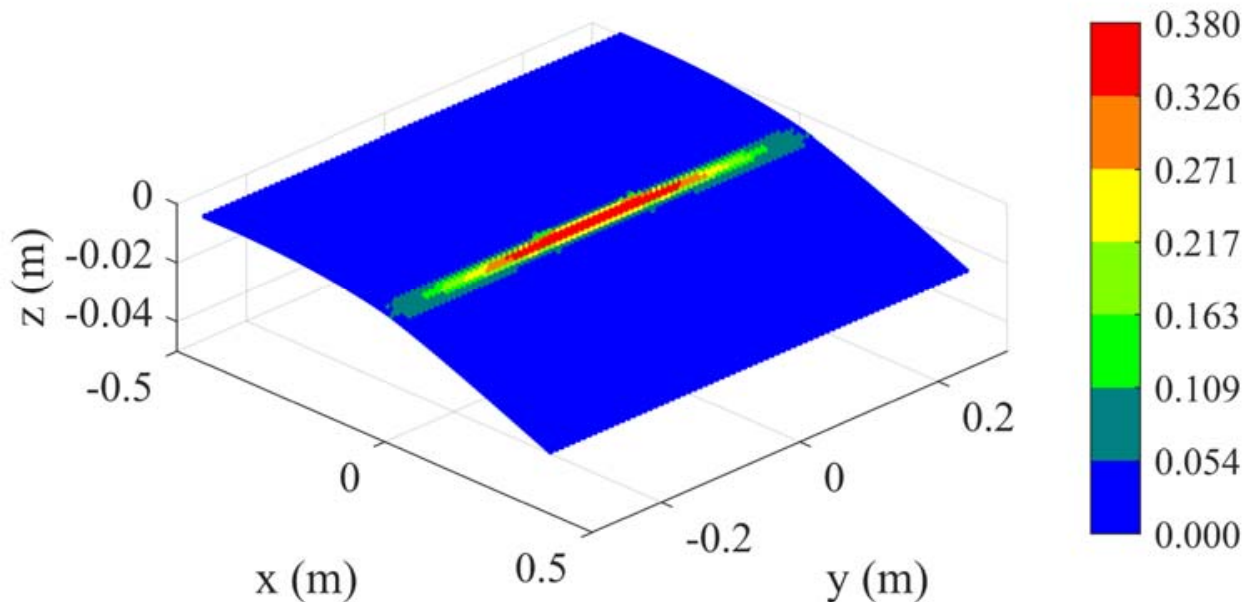

(d)

Fig. 43. Crack propagation for Case 1 when: (a) $w=0$, (b) $w=-0.0351 \mathrm{~m}$, (c) $w=-0.0359 \mathrm{~m}$, (d) $w=-0.0366 \mathrm{~m}$

Fig. 44 presents damage evolution on the flat shell with initial crack orientation $\beta=45^{\circ}$ (case 2). The initial damage on the flat shell is shown in Fig. 44(a). The damage evolution on the flat shell when the applied displacements $w=-0.0321 \mathrm{~m},-0.0328 \mathrm{~m},-0.0335 \mathrm{~m}$ are shown in Figs. $44(\mathrm{~b}, \mathrm{c}, \mathrm{d})$, respectively. As can be seen from the figures, the crack propagates only on one side of the crack tip which is near the applied loading edge. As the applied displacement increases, the crack continues propagating on this side and reaches the boundary edge of the flat shell when the applied displacement is $w=-0.0335 \mathrm{~m}$. It can also observe that although the initial orientation of the crack is $\beta=45^{\circ}$, the newly propagated crack path is almost parallel to the right edge on which the incremental displacements are applied. 


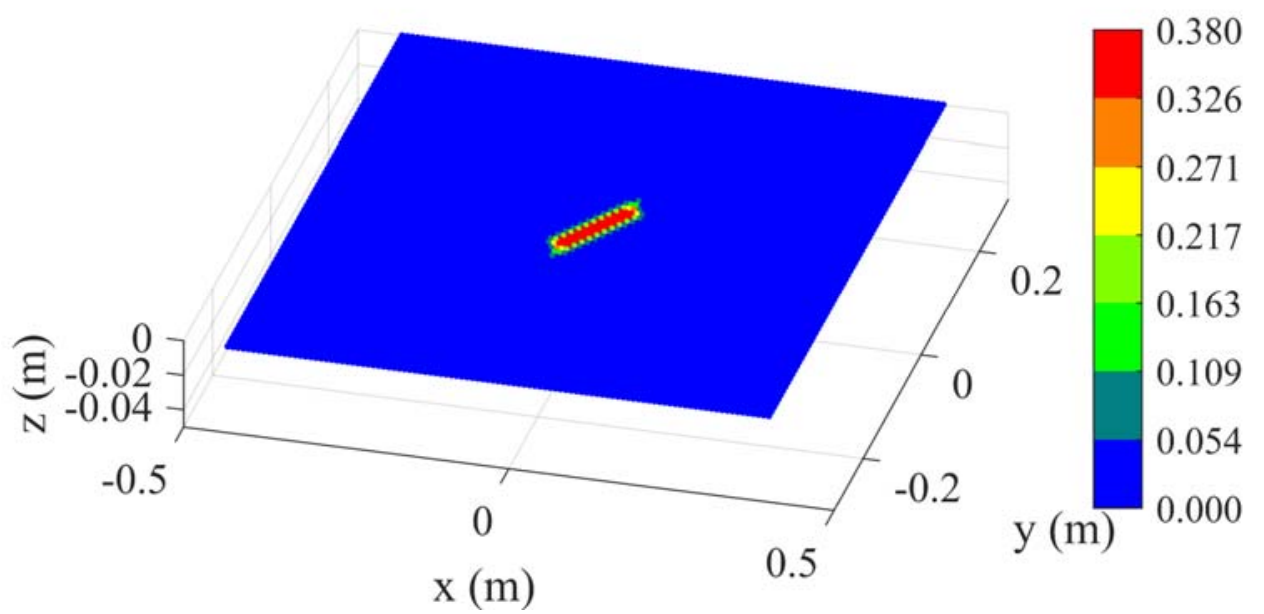

(a)

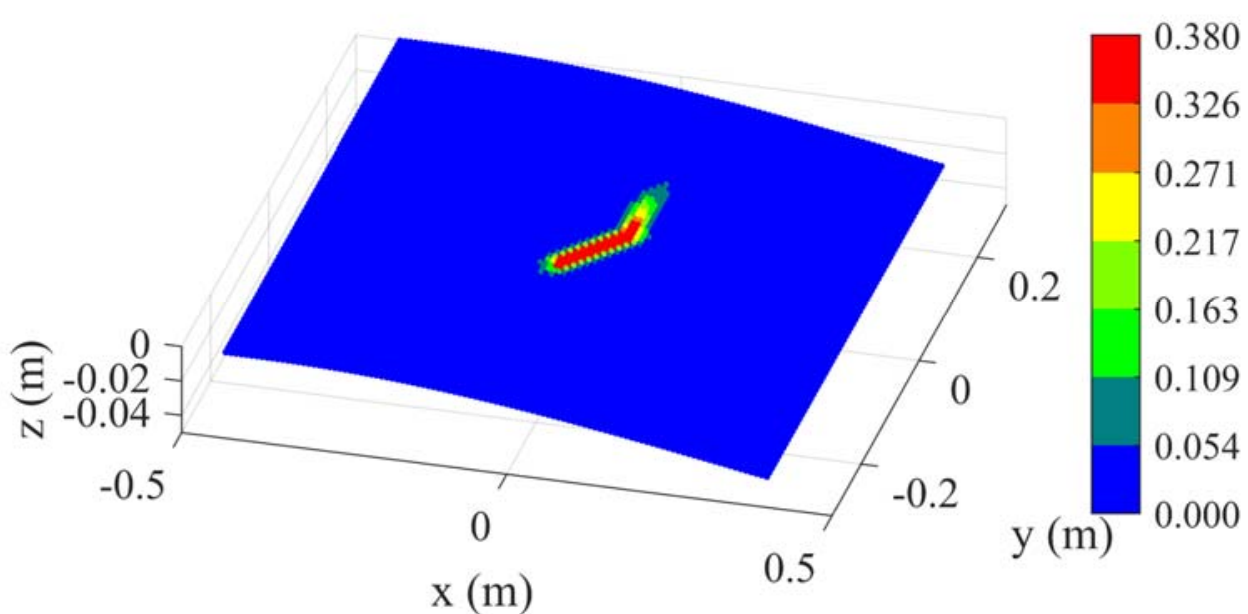

(b)

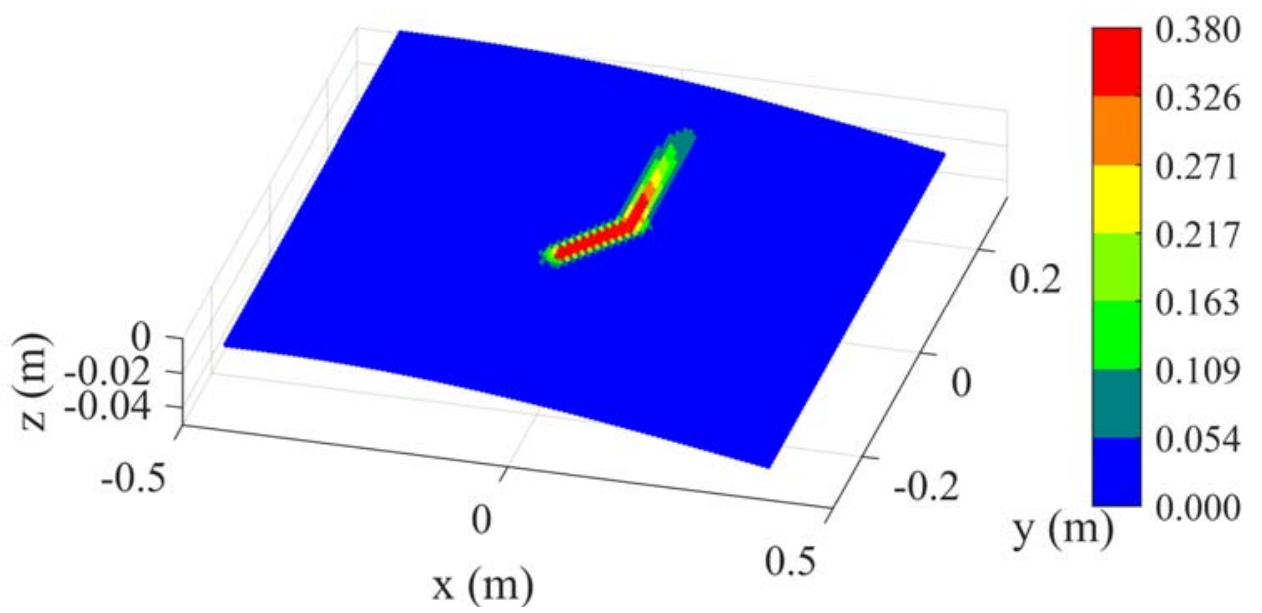

(c) 


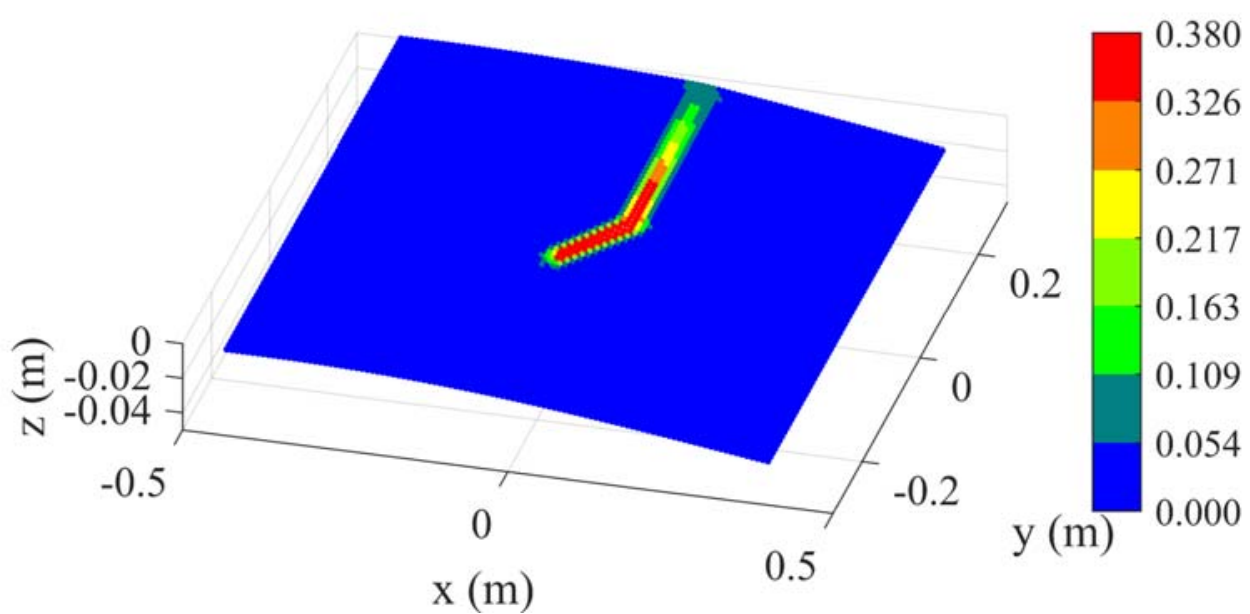

(d)

Fig. 44. Crack propagation for Case 2 when: (a) $w=0$, (b) $w=-0.0321 \mathrm{~m}$, (c) $w=-0.0328 \mathrm{~m}$, (d) $w=-0.0335 \mathrm{~m}$

7.6. Damage prediction of the glass cup with hot water In this section, the damage occurrence in a glass cup due to sudden temperature change is investigated. It is assumed that the lower part of the cup, shown in red, is subjected to $\Delta T=80^{\circ} \mathrm{C}$ temperature change and the upper part of the cup, shown in blue, subjected to $\Delta T=0^{\circ} \mathrm{C}$ temperature change as shown in Fig. 45(a). The shape of the cup is defined as

$x=b \cos \theta \cos \phi$

$y=b \cos \theta \sin \phi$

$z=(a+e \sin \theta) \sin \theta$

where $a=0.1 \mathrm{~m}, b=0.06 \mathrm{~m}, e=0.04 \mathrm{~m},-\pi / 2 \leq \theta \leq \pi / 12,-\pi \leq \phi \leq \pi$

The cup is assumed to have a randomly distributed thickness as shown in Fig. 45(b) as

$h(z)= \begin{cases}h_{0}\left(2-\left|\frac{z+0.06}{0.0039}\right|\right) & \text { for }-0.06 \leq z \leq-0.0561 \\ h_{0} f & \text { for }-0.0561<z \leq 0.02625\end{cases}$

where $0.9 \leq f \leq 1.1$ and $h_{0}=1 \times 10^{-3} \mathrm{~m}$.

The water level inside the cup is assumed as $d_{\text {water }}=0.0345 \mathrm{~m}$ measure from bottom of the cup. The material has Young's modulus $E=68 \times 10^{9} \mathrm{~N} / \mathrm{m}^{2}$, Poisson's ratio $v=0.17$, mass density $\rho=2710 \mathrm{~kg} / \mathrm{m}^{3}$ and linear thermal expansion coefficient $\alpha=8.5 \times 10^{-6} \mathrm{~m} / \mathrm{mK}$ [42]. The critical energy release rate of the glass is taken as $G_{c}=5.2941 \mathrm{~J} / \mathrm{m}^{2}[42]$. 


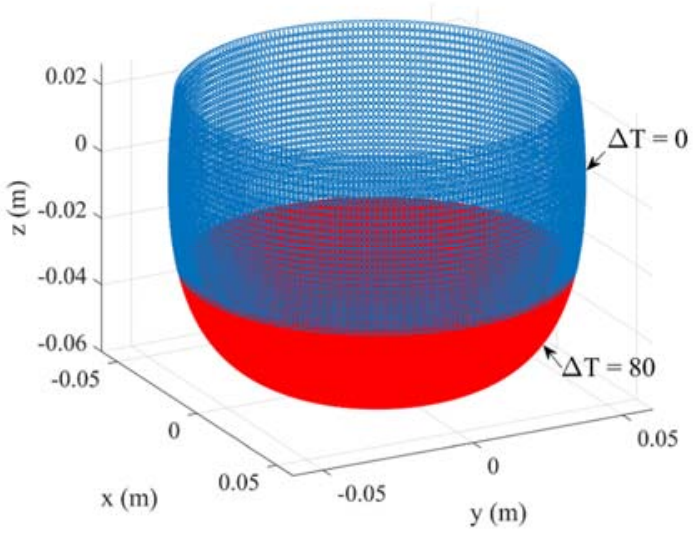

(a)

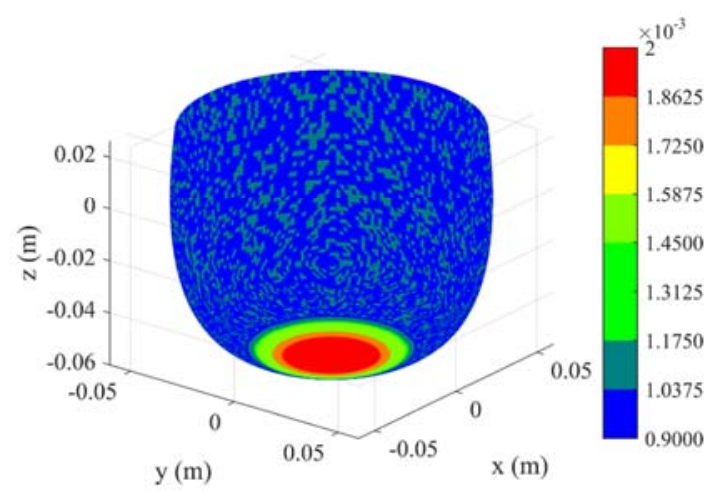

(b)

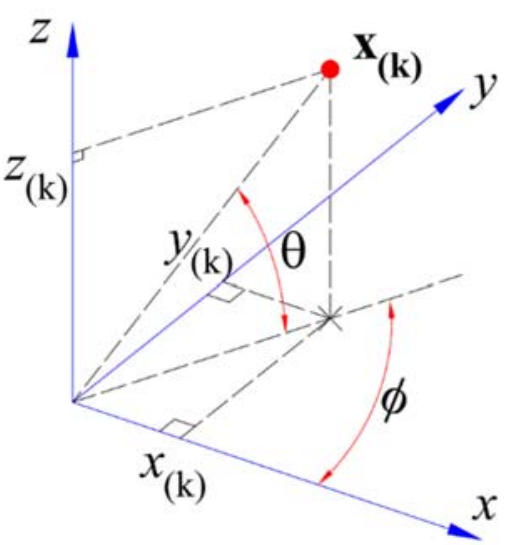

(c)

Fig. 45. Glass cup with hot water (a) 3D model (b) random thickness variation,(m) (c) coordinate system

Since the cup is applied sudden temperature change, the problem is solved dynamically by using explicit time integration scheme with time step size of $d t=0.01 \mu \mathrm{s}$. The PD model is discretized by setting incremental angles as $d \theta=\pi / 120$ and $d \phi=\pi / 90$.

Figs. 46-47 present the extent of the damage coefficient, $\phi$ on the cup. As shown in Fig. 46(a), the cup initially gets damaged around the interface region between the hot and cold part of the cup. Later, the cup gets damaged around the interface region where there is a sudden thickness change as shown in Fig. 46(b). As the time increases, the extent of damage is increased in these two regions and, the cup is almost split into three parts at $t=4.5 \mu \mathrm{s}$ as shown in Fig. 47. 


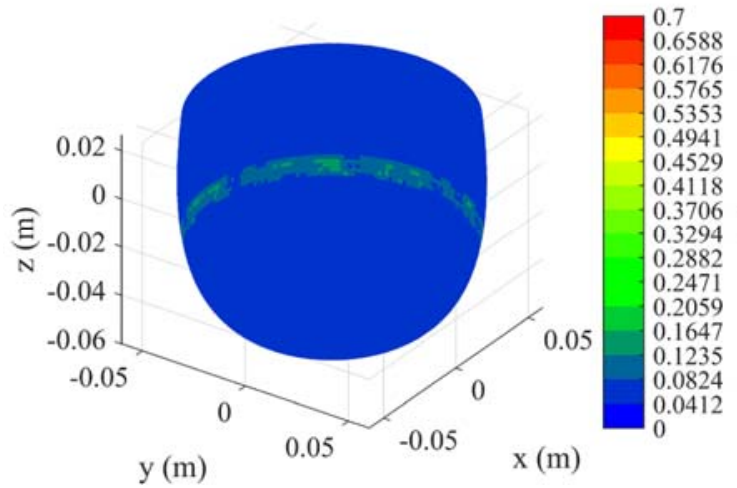

(a)

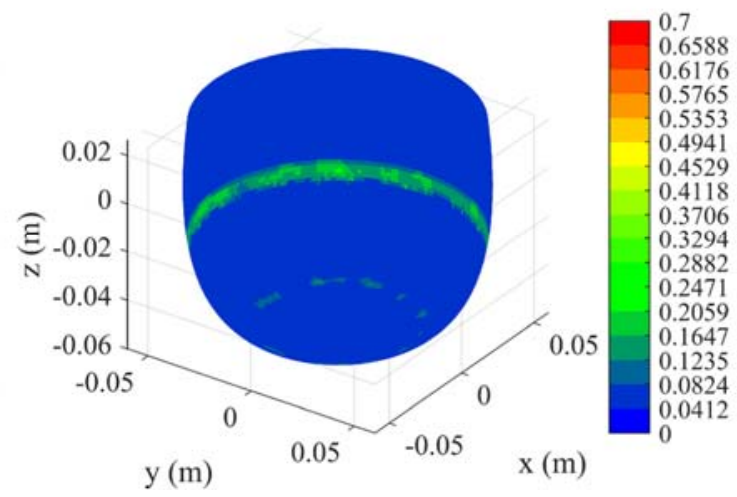

(b)

Fig. 46. Variation of damage coefficient, $\phi$ at (a) $t=2 \mu \mathrm{s}$, (b) $t=2.5 \mu \mathrm{s}$
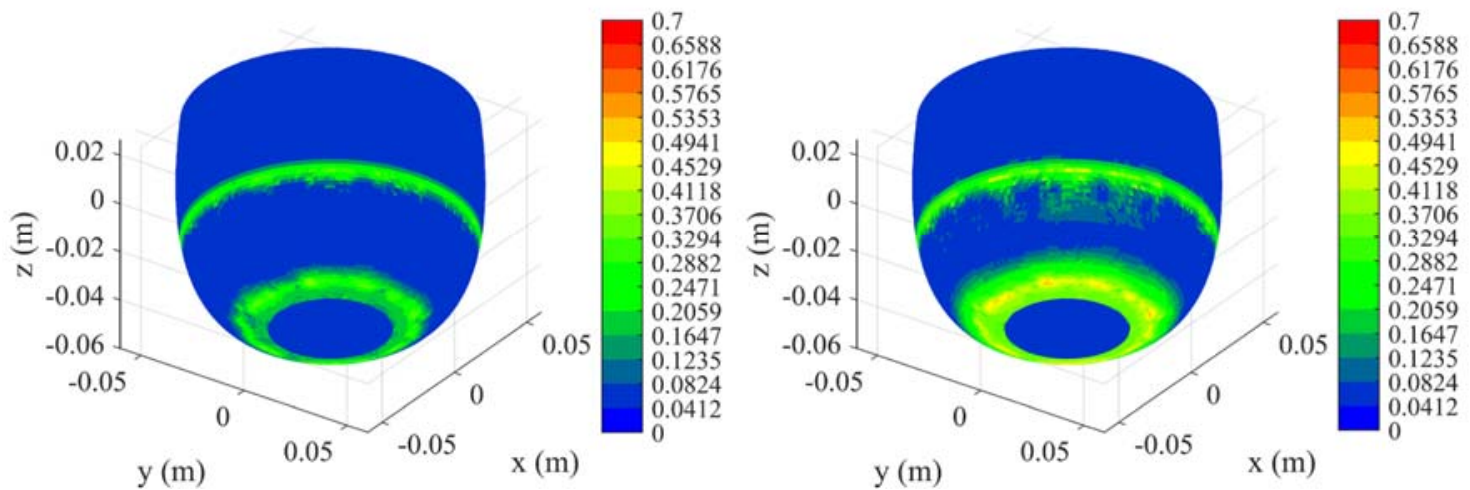

Fig. 47. Variation of damage coefficient, $\phi$ at (a) $t=3.5 \mu \mathrm{s}$, (b) $t=4.5 \mu \mathrm{s}$

7.7. Damage prediction for a dropped egg

In this example, the extent of damage in a dropped egg is investigated. The shape of the typical chicken egg is defined as [43]

$y=\frac{W}{2} \cos \theta \cos \phi$

$z=\frac{W}{2} \cos \theta \sin \phi$

$x=\left(\frac{H}{2}+e \sin \theta\right) \sin \theta$

with

$-\pi / 2 \leq \theta \leq \pi / 2,-\pi \leq \phi \leq \pi$

where $H$ represents the height and $W$ represents the width of the egg. In Eq. (62c), e represents the egg parameter. The egg parameter is chosen as $e=0.004 \mathrm{~m}$ to modify the shape of an ellipsoid to the shape of an egg [43]. The height and width of the egg is chosen as $H=0.06 \mathrm{~m}$ and $W=0.045 \mathrm{~m}$, respectively.

The material properties of the eggshell are described as follows: Elastic modulus, $E=55 \times 10^{9} \mathrm{~N} / \mathrm{m}^{2}$ [44], Poisson's ratio, $v=0.35$ [45], mass density $\rho=2050 \mathrm{~kg} / \mathrm{m}^{3}$ [46], critical energy release rate, $G_{c}=1.6364 \mathrm{~J} / \mathrm{m}^{2}[47]$. 
It is assumed that the eggshell falls from a height of $0.2225 \mathrm{~m}$ as shown in Fig. 48(a). The density of the fluid inside the eggshell is considered as $\rho_{\text {fluid }}=1024.3 \mathrm{~kg} / \mathrm{m}^{3}$ [48], the weight of the fluid is taken as $0.0652 \mathrm{~kg}$ and the weight of the eggshell is ignored. In order to represent the heterogeneity of the eggshell, a randomly distributed thickness is assumed for each material point in a range of $3.325 \times 10^{-4} \mathrm{~m} \leq h \leq 3.675 \times 10^{-4} \mathrm{~m}$ as shown in Fig. 49.

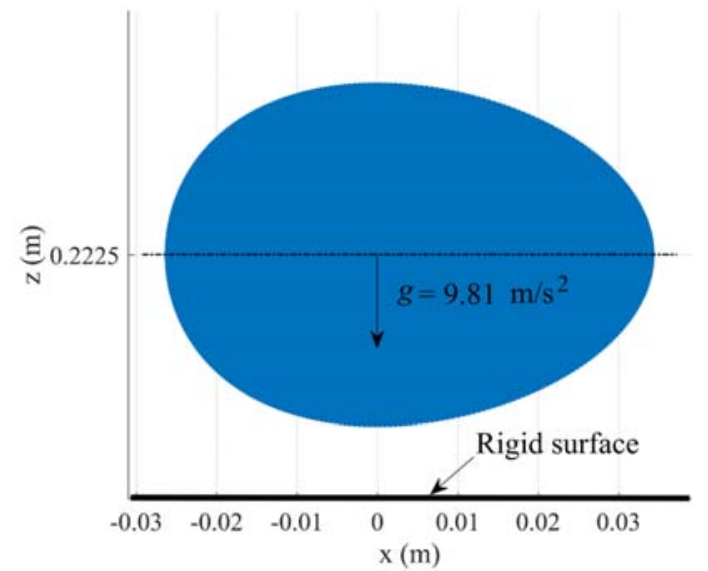

(a)

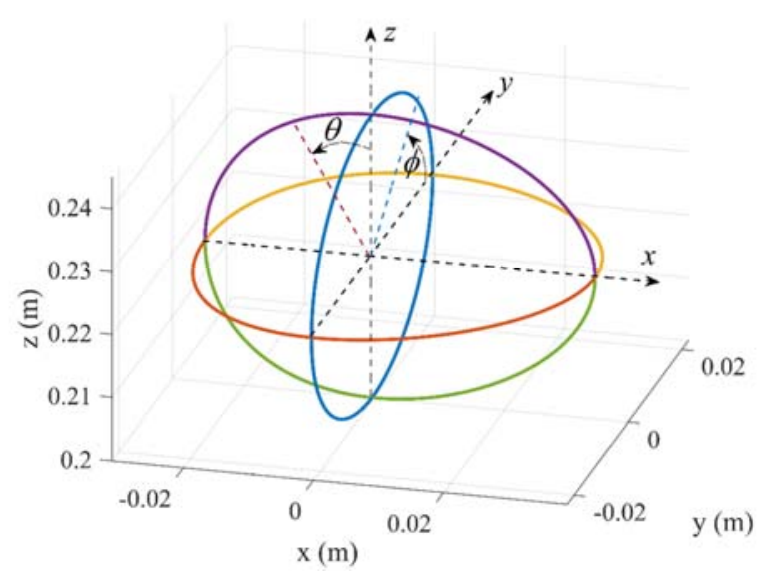

(b)

Fig. 48. Eggshell (a) geometry (b) coordinate system

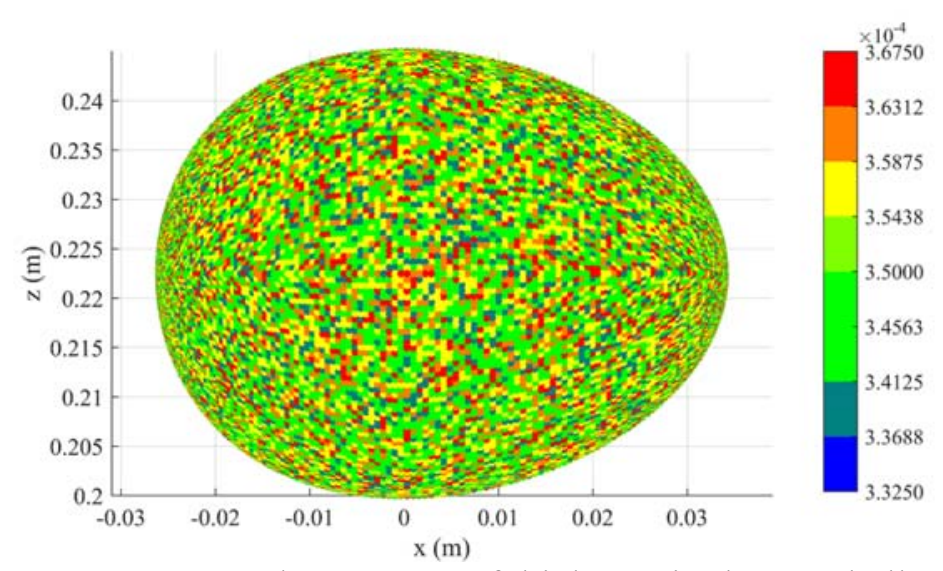

Fig. 49. Random scatter of thickness in the eggshell

When the eggshell hits the rigid surface at $t=0.2019 \mathrm{~s}$, the velocity of the egg is calculated as $\mathrm{v}_{\text {fluid }}=-1.9809 \mathrm{~m} / \mathrm{s}$. It is assumed that after $100 \mu \mathrm{s}$, the fluid velocity inside the egg reduces to $\mathrm{v}_{\text {fluid }}=0$. Therefore, the acceleration of the fluid inside the eggshell is calculated as

$a_{\text {fluid }}=\frac{0-(-1.9809)}{100 \times 10^{-6}}=1.9809 \times 10^{4} \mathrm{~m} / \mathrm{s}^{2}$

It is assumed that the pressure on egg surface area, $A_{(k)}$ is caused by inertia forces of fluid particles that are vertically aligned with material point $k$ as shown in Fig. 50 and all of the fluid particles 
have the same acceleration as provided in Eq. (63). The total inertial force acting on surface $A_{k \text { ) }}$ can be calculated as

$\vec{F}_{z(k)}=-\rho_{\text {fluid }} V_{\text {fluid }(k)} \vec{a}_{\text {fluid }}$

where $V_{\text {fluid }(k)}$ represents the volume of the fluid particles that are vertically aligned with material point $k$. Therefore, the normal force acting on surface $A_{(k)}$ is evaluated as

$\left|\vec{F}_{n(k)}\right|=\left|\vec{F}_{z(k)} / c_{3(k)}\right|$

$\vec{F}_{n(k)}=\left|\vec{F}_{n(k)}\right| \vec{n}_{z^{\prime}(k)}$

where $\vec{n}_{z^{\prime}(k)}$ represents the normal vector of surface $A_{(k)}$ as

$\vec{n}_{\mathbf{\prime}^{\prime}(k)}=\left[\begin{array}{lll}C_{1(k)} & C_{2(k)} & C_{3(k)}\end{array}\right]^{T}$

Therefore, the pressure generated on the surface $A_{(k)}$ can be estimated as

$\vec{p}_{n(k)}=\vec{F}_{n(k)} / A_{(k)}$

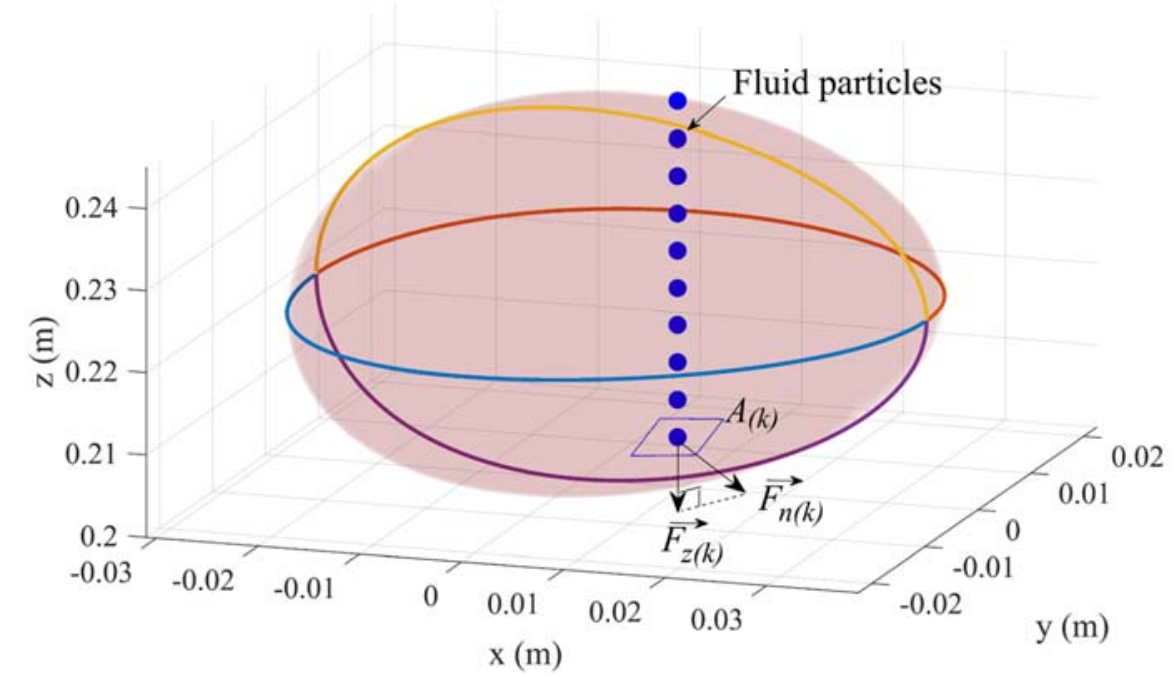

Fig. 50. Calculating pressure on eggshell due to inside fluid

Fig. 51 shows the distribution of pressure acting on the eggshell due to the inertial forces of fluid inside the egg. As can be seen from the figure, only the lower half of the eggshell is subjected to pressure caused by the fluid. 


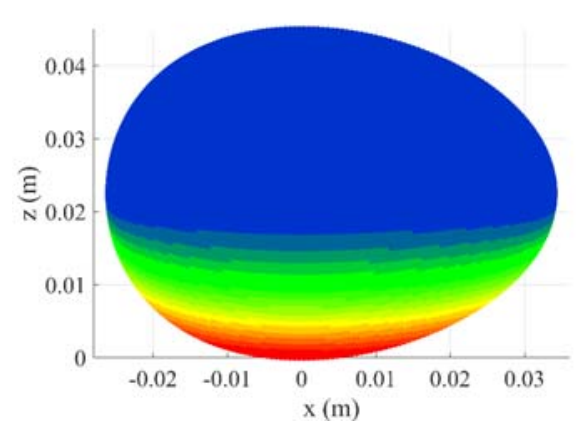

(a)
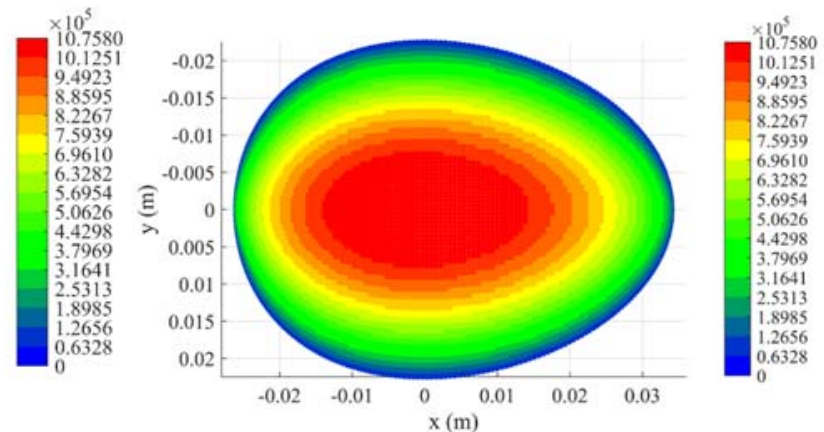

(b)

Fig. 51. Pressure distribution, $(\mathrm{Pa})$ on egg surface due to inertial forces (a) side view (b) bottom view of the eggshell

Similar to previous example, the damage process of the eggshell is simulated by using explicit time integration scheme with time step size of $d t=0.01 \mu \mathrm{s}$. Note that, during the simulations, the locations of the material points are updated. If vertical coordinate of a material point is $z \leq 0$, it is updated as $z=0$, where the rigid surface is located.

Figs. 52-55 show the extent of damage on the eggshell during falling at different times. As the time progresses, the extent of the damage increases. The material points with damage coefficient $\phi \geq 0.37$ represent the completely split region on the eggshell. The damaged region is continuously expanded, and the eggshell is split into many pieces as shown in Figs. 53-55. As shown in Fig. 55, after $t=10 \mu$ s the lower part of the eggshell is destroyed into many small pieces and the damage region expanded to the upper part of the eggshell.

Fig. 56 shows the comparison of damage for a dropped egg with PD predictions. As it can be observed from the figure, the damage patterns predicted in PD simulation are similar to the experimental results. However, it can also be seen from the figure that the fragments of the experiment shown in Fig. 56(a) are larger than the PD results shown in Fig. 56(b). The difference can be explained as follows:

First, in this example, the pressures generated on the eggshell are calculated based on the simplified fluid model inside the eggshell. Therefore, more realistic predictions can be obtained with more realistic computational fluid dynamic (CFD) simulations. Moreover, in this example, fluidstructure interaction between the eggshell and egg liquid is not fully coupled. Therefore, coupling CFD simulation and PD prediction can be further investigated.

Second, although the material properties of the eggshell are chosen based on recent experimental studies, the eggshell is modelled with one layer of isotropic material. In fact, the eggshell consists of many layers with different material properties [49]. Therefore, better predictions can be obtained with more complex material models. 


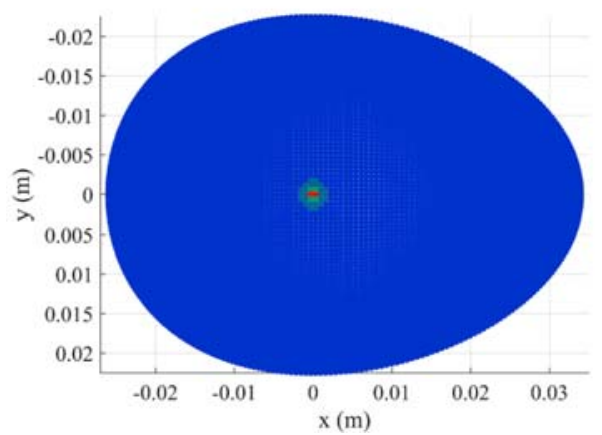

(a)

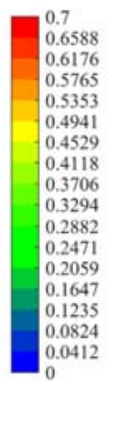

(b)

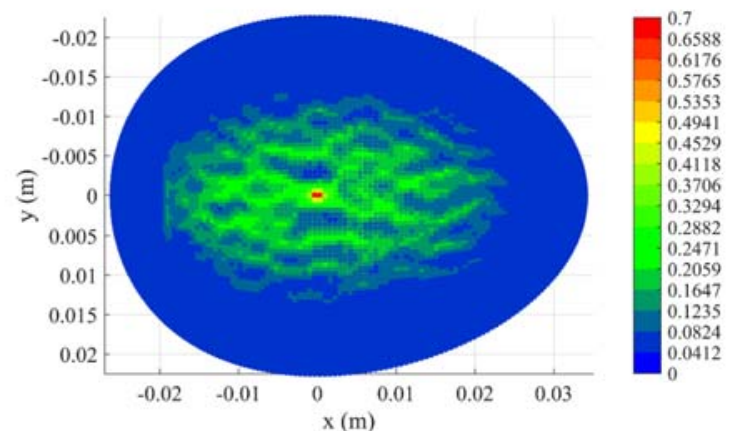

Fig. 52. The extent of damage at (a) $t=2.5 \mu \mathrm{s}$ (b) $t=3.75 \mu \mathrm{s}$ after egg hits the surface

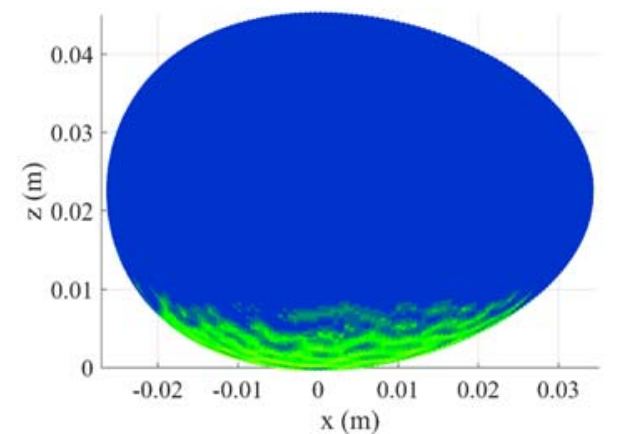

(a)
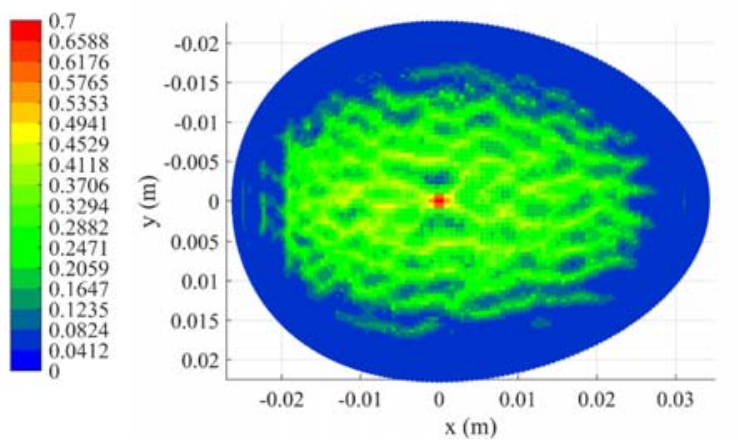

(b)

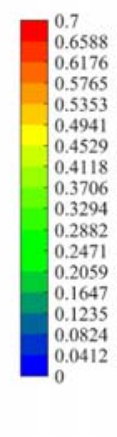

Fig. 53. The extent of damage at $t=5 \mu \mathrm{s}$ after egg hits the surface (a) side view (b) bottom view

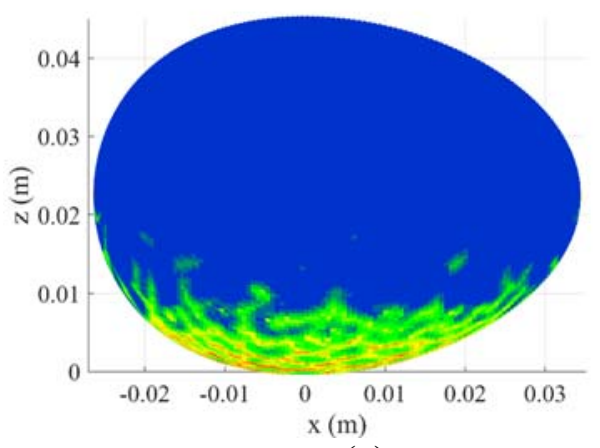

(a)

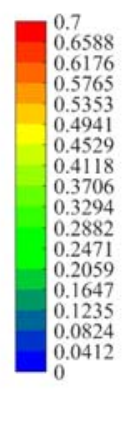

(b)

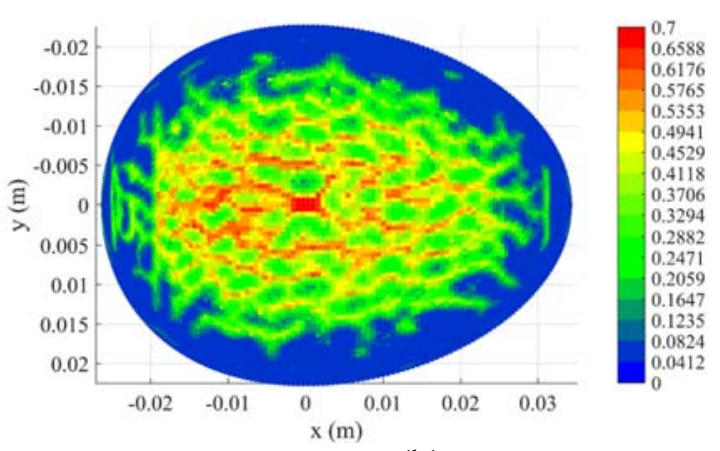

Fig. 54. The extent of damage at $t=7.5 \mu$ s after egg hits the surface (a) side view (b) bottom view 


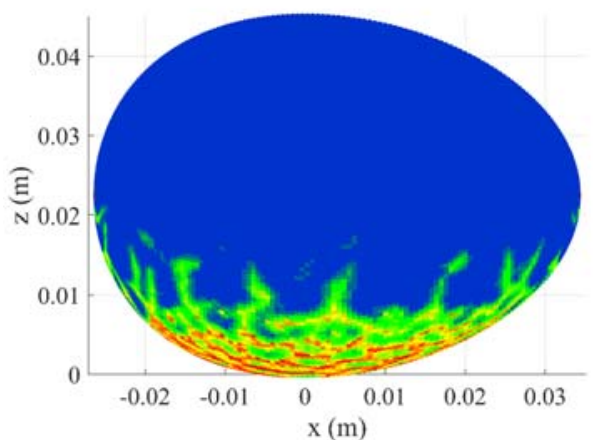

(a)

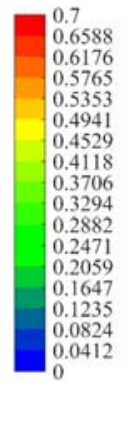

(b)

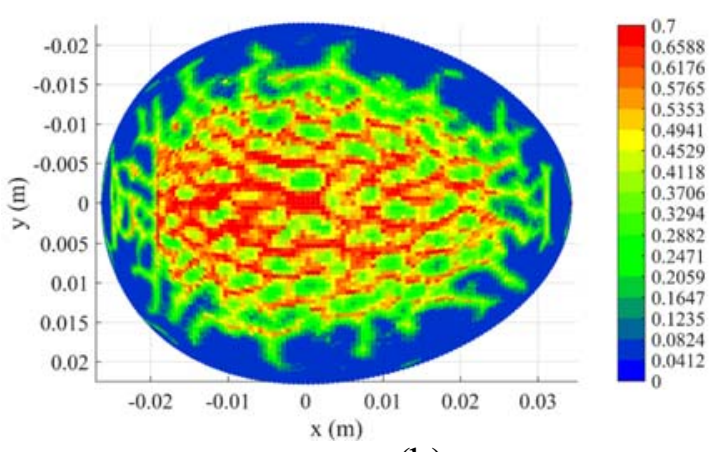

Fig. 55. The extent of damage at $t=10 \mu \mathrm{s}$ after egg hits the surface (a) side view (b) bottom view

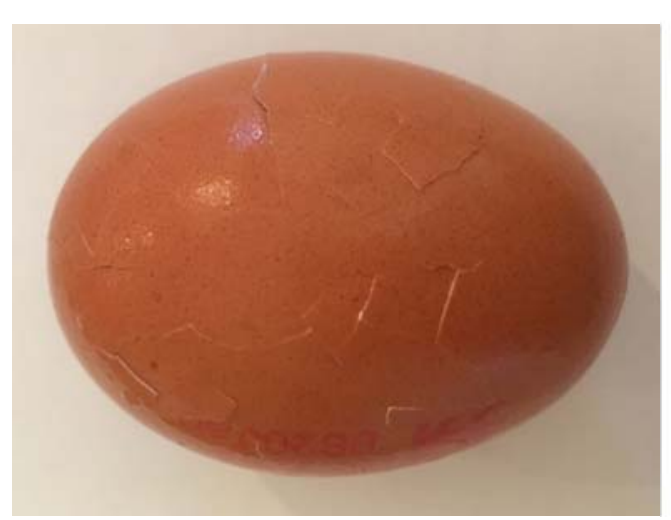

(a)

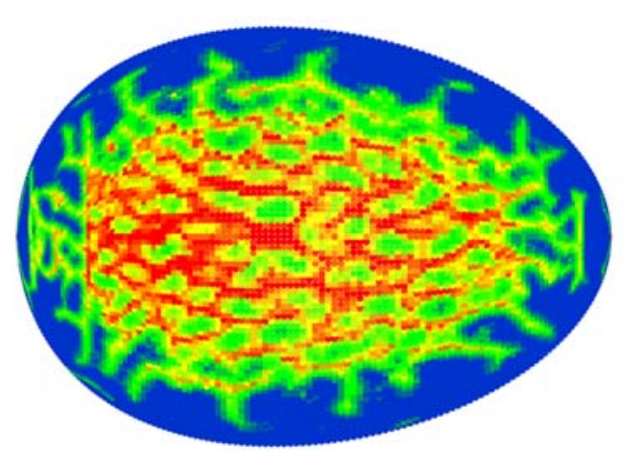

(b)

Fig. 56. Damage for (a) a dropped egg (b) PD predictions

\section{Concluding remarks}

This study presents a novel state-based peridynamic model for the thermomechanical behavior of shell structures in three-dimensional space with 6 degrees of freedom. The PD model is firstly developed for flat shells, and then modified for curve shells, and further developed for stiffened structures. The numerical procedure to deal with complex shell structures is also provided. The behaviors of shell structures captured by the developed PD model are verified by comparing with FEA solutions with very good agreements. The PD damage criteria for shell structures based on critical energy release rate is provided for damage predictions.

The developed PD model is used to predict damage on a flat shell in double torsion problem, a flat shell with pre-existing crack, a dropped egg and a glass cup subjected to temperature change. The developed PD model can be used for any type of shell structures to predict possible damages that may occur during the operation process.

\section{Acknowledgment}

The authors gratefully acknowledge financial support from the Ministry of Education and Training in Vietnam and University of Strathclyde. 


\section{Appendix A. Peridynamic constants for in-plane deformations}

A.1. PD dilatation constant, $d_{i p}$

By using Taylor's series expansion and by ignoring the higher order terms, the displacement components of material point $j$ can be expressed as

$$
\begin{aligned}
& u_{(j)}^{\prime}=u_{(k)}^{\prime}+u_{, x^{\prime}(k)}^{\prime}\left(x_{(j)}^{\prime}-x_{(k)}^{\prime}\right)+u_{, y^{\prime}(k)}^{\prime}\left(y_{(j)}^{\prime}-y_{(k)}^{\prime}\right) \\
& v_{(j)}^{\prime}=v_{(k)}^{\prime}+v_{, x^{\prime}(k)}^{\prime}\left(x_{(j)}^{\prime}-x_{(k)}^{\prime}\right)+v_{, y^{\prime}(k)}^{\prime}\left(y_{(j)}^{\prime}-y_{(k)}^{\prime}\right) \\
& w_{(j)}^{\prime}=w_{(k)}^{\prime}+w_{, x^{\prime}(k)}^{\prime}\left(x_{(j)}^{\prime}-x_{(k)}^{\prime}\right)+w_{, y^{\prime}(k)}^{\prime}\left(y_{(j)}^{\prime}-y_{(k)}^{\prime}\right)
\end{aligned}
$$

The relations given in Eq. (A.1) can be rewritten as

$\frac{u_{(j)}^{\prime}-u_{(k)}^{\prime}}{\xi}=u_{, x^{\prime}(k)}^{\prime} \cos \varphi+u_{, y^{\prime}(k)}^{\prime} \sin \varphi$

$\frac{v_{(j)}^{\prime}-v_{(k)}^{\prime}}{\xi}=v_{, x^{\prime}(k)}^{\prime} \cos \varphi+v_{, y^{\prime}(k)}^{\prime} \sin \varphi$

$\frac{w_{(j)}^{\prime}-w_{(k)}^{\prime}}{\xi}=w_{, x^{\prime}(k)}^{\prime} \cos \varphi+w_{, y^{\prime}(k)}^{\prime} \sin \varphi$

Therefore, by using Eq. (A.2a) and (A.2b), the bond stretch for in-plane deformations given in Eq. (24) can be rewritten as

$s_{i p(k)(j)}=u_{, x^{\prime}(k)}^{\prime} \cos ^{2} \varphi+u_{, y^{\prime}(k)}^{\prime} \sin \varphi \cos \varphi+v_{, x^{\prime}(k)}^{\prime} \sin \varphi \cos \varphi+v_{, y^{\prime}(k)}^{\prime} \sin ^{2} \varphi$

The dilatation for in-plane deformations given in Eq. (22) can be rewritten in an integral form as

$\vartheta_{(k)}=d_{i p} h \int_{0}^{\delta} \int_{0}^{2 \pi}\left(\begin{array}{l}u_{, x^{\prime}(k)}^{\prime} \cos ^{2} \varphi+u_{, y^{\prime}(k)}^{\prime} \sin \varphi \cos \varphi \\ +v_{, x^{\prime}(k)}^{\prime} \sin \varphi \cos \varphi+v_{, y^{\prime}(k)}^{\prime} \sin ^{2} \varphi-\alpha \Delta T_{(k)}\end{array}\right) \xi d \xi d \varphi+2 \alpha \Delta T_{(k)}$

By performing integrations given in Eq. (A.4), the dilatation for in-plane deformations can be calculated as

$$
\vartheta_{(k)}=d_{i p} \frac{\pi h \delta^{2}}{2}\left(u_{, x^{\prime}(k)}^{\prime}+v_{, y^{\prime}(k)}^{\prime}\right)+\alpha \Delta T_{(k)}\left(2-d_{i p} \pi h \delta^{2}\right)
$$

Meanwhile, the classical form of dilatation can be described as $\vartheta_{(k)}^{C C M}=u_{, x^{\prime}(k)}^{\prime}+v_{, y^{\prime}(k)}^{\prime}$

By comparing Eq. (A.5) and (A.6), the PD dilatation constant $d_{i p}$ can be defined as

$d_{i p}=\frac{2}{\pi h \delta^{2}}$

A.2. PD material constants, $a_{i p 1}, a_{i p 2}, a_{i p 3}$ and $b_{i p}$

By using the stretch definition in Eq. (A.3) and dilatation term in Eq. (A.6), the strain energy per unit area for in-plane deformations given in Eq. (20) can be rewritten in an integral form as 


$$
\begin{aligned}
\bar{W}_{\text {inplane }(k)}^{P D}= & a_{i p 1}\left(u_{, x^{\prime}(k)}^{\prime}+v_{, y^{\prime}(k)}^{\prime}\right)^{2}-a_{i p 2}\left(u_{, x^{\prime}(k)}^{\prime}+v_{, y^{\prime}(k)}^{\prime}\right) \Delta T_{(k)}+a_{i p 3} \Delta T_{(k)}^{2} \\
& +b_{i p} h \int_{0}^{\delta} \int_{0}^{2 \pi}\left(\begin{array}{l}
u_{, x^{\prime}(k)}^{\prime} \cos ^{2} \varphi+u_{, y^{\prime}(k)}^{\prime} \sin \varphi \cos \varphi \\
+v_{, x^{\prime}(k)}^{\prime} \sin \varphi \cos \varphi+v_{, y^{\prime}(k)}^{\prime} \sin ^{2} \varphi-\alpha \Delta T_{(k)}
\end{array}\right)^{2} \xi^{2} d \xi d \varphi
\end{aligned}
$$

After performing integrations given in Eq. (A.8), the strain energy per unit area for the in-plane deformations can be evaluated as

$$
\begin{aligned}
\bar{W}_{\text {inplane }(k)}^{P D}= & a_{i p 1}\left(u_{, x^{\prime}(k)}^{\prime}+v_{, y^{\prime}(k)}^{\prime}\right)^{2}-a_{i p 2}\left(u_{, x^{\prime}(k)}^{\prime}+v_{, y^{\prime}(k)}^{\prime}\right) \Delta T_{(k)}+a_{i p 3} \Delta T_{(k)}^{2} \\
& +b_{i p} \frac{\pi h \delta^{3}}{12}\left(3 u_{, x^{\prime}(k)}^{\prime 2}+3 v_{, y^{\prime}(k)}^{\prime 2}+u_{, y^{\prime}(k)}^{\prime 2}+v_{, x^{\prime}(k)}^{\prime 2}+2 u_{, x^{\prime}(k)}^{\prime} v_{, y^{\prime}(k)}^{\prime}+2 u_{, y^{\prime}(k)}^{\prime} v_{, x^{\prime}(k)}^{\prime}\right) \\
& +b_{i p} \frac{2 \pi h \delta^{3}}{3}\left[-\alpha \Delta T_{(k)}\left(u_{, x^{\prime}(k)}^{\prime}+v_{, y^{\prime}(k)}^{\prime}\right)+\alpha^{2} \Delta T_{(k)}^{2}\right]
\end{aligned}
$$

or

$$
\begin{aligned}
\bar{W}_{\text {inplane }(k)}^{P D}= & a_{i p 1}\left(u_{, x^{\prime}(k)}^{\prime}+v_{, y^{\prime}(k)}^{\prime}\right)^{2} \\
& +b_{i p} \frac{\pi h \delta^{3}}{12}\left[3\left(u_{, x^{\prime}(k)}^{\prime}+v_{, y^{\prime}(k)}^{\prime}\right)^{2}-4 u_{, x^{\prime}(k)}^{\prime} v_{, y^{\prime}(k)}^{\prime}+\left(u_{, y^{\prime}(k)}^{\prime}+v_{, x^{\prime}(k)}^{\prime}\right)^{2}\right] \\
& -\left(a_{i p 2}+b_{i p} \frac{2 \pi h \delta^{3}}{3} \alpha\right)\left(u_{, x^{\prime}(k)}^{\prime}+v_{, y^{\prime}(k)}^{\prime}\right) \Delta T_{(k)} \\
& +\left(a_{i p 3}+b_{i p} \frac{2 \pi h \delta^{3}}{3} \alpha^{2}\right) \Delta T_{(k)}^{2}
\end{aligned}
$$

By comparing Eq. (A.10) and (14b) following relations can be obtained

$$
\begin{aligned}
& b_{i p} \frac{\pi h \delta^{3}}{12}=\frac{E h}{4(1+v)} \\
& a_{i p 1}+b_{i p} \frac{\pi h \delta^{3}}{4}=\frac{E h}{2\left(1-v^{2}\right)} \\
& a_{i p 2}+b_{i p} \frac{2 \pi h \delta^{3}}{3} \alpha=\frac{E h}{1-v} \alpha \\
& a_{i p 3}+b_{i p} \frac{2 \pi h \delta^{3}}{3} \alpha^{2}=\frac{E h}{1-v} \alpha^{2}
\end{aligned}
$$

Finally, by using the relations in Eq. (A.11), the PD constants for in-plane deformations can be defined as

$$
\begin{aligned}
& b_{i p}=\frac{3 E}{\pi \delta^{3}(1+v)} \\
& a_{i p 1}=\frac{E h(3 v-1)}{4\left(1-v^{2}\right)} \\
& a_{i p 2}=\frac{E h \alpha}{1-v^{2}}(3 v-1)
\end{aligned}
$$


$a_{i p 3}=\frac{E h}{1-v^{2}}(3 v-1) \alpha^{2}$

\section{Appendix B. Peridynamic constants for bending deformations}

B.1. PD constant, $d_{b}$

By using Taylor's series expansion and by ignoring the higher order terms, the rotation components of material point $j$ can be expressed as

$$
\begin{aligned}
& \theta_{x^{\prime}(j)}=\theta_{x^{\prime}(k)}+\theta_{x^{\prime}, x^{\prime}(k)}\left(x_{(j)}^{\prime}-x_{(k)}^{\prime}\right)+\theta_{x^{\prime}, y^{\prime}(k)}\left(y_{(j)}^{\prime}-y_{(k)}^{\prime}\right) \\
& \theta_{y^{\prime}(j)}=\theta_{y^{\prime}(k)}+\theta_{y^{\prime}, x^{\prime}(k)}\left(x_{(j)}^{\prime}-x_{(k)}^{\prime}\right)+\theta_{y^{\prime}, y^{\prime}(k)}\left(y_{(j)}^{\prime}-y_{(k)}^{\prime}\right)
\end{aligned}
$$

The relations given in Eq. (B.1) can be rewritten as

$$
\begin{aligned}
& \frac{\theta_{x^{\prime}(j)}-\theta_{x^{\prime}(k)}}{\xi}=\theta_{x^{\prime}, x^{\prime}(k)} \cos \varphi+\theta_{x^{\prime}, y^{\prime}(k)} \sin \varphi \\
& \frac{\theta_{y^{\prime}(j)}-\theta_{y^{\prime}(k)}}{\xi}=\theta_{y^{\prime}, x^{\prime}(k)} \cos \varphi+\theta_{y^{\prime}, y^{\prime}(k)} \sin \varphi
\end{aligned}
$$

Therefore, by using Eq. (B.2), the term $S_{b(k)(j)}$ given in Eq. (27b) can be rewritten as

$$
s_{b(k)(j)}=-\theta_{y^{\prime}, x^{\prime}(k)} \cos ^{2} \varphi-\theta_{y^{\prime}, y^{\prime}(k)} \sin \varphi \cos \varphi+\theta_{x^{\prime}, x^{\prime}(k)} \sin \varphi \cos \varphi+\theta_{x^{\prime}, y^{\prime}(k)} \sin ^{2} \varphi
$$

The term $\vartheta_{b(k)}$ given in Eq. (27a) can be rewritten in an integral form as

$$
\vartheta_{b(k)}=d_{b} h \int_{0}^{\delta} \int_{0}^{2 \pi}\left(\begin{array}{l}
-\theta_{y^{\prime}, x^{\prime}(k)} \cos ^{2} \varphi-\theta_{y^{\prime}, y^{\prime}(k)} \sin \varphi \cos \varphi \\
+\theta_{x^{\prime}, x^{\prime}(k)} \sin \varphi \cos \varphi+\theta_{x^{\prime}, y^{\prime}(k)} \sin ^{2} \varphi
\end{array}\right) \xi d \xi d \varphi
$$

By performing integrations given in Eq. (B.4), the term $\vartheta_{b(k)}$ can be written as

$$
\vartheta_{b(k)}=d_{b} \frac{\pi h \delta^{2}}{2}\left(-\theta_{y^{\prime}, x^{\prime}(k)}+\theta_{x^{\prime}, y^{\prime}(k)}\right)
$$

On the other hand, the corresponding term in classical form can be described as

$$
\vartheta_{b(k)}^{C C M}=-\theta_{y^{\prime}, x^{\prime}(k)}+\theta_{x^{\prime}, y^{\prime}(k)}
$$

By comparing Eq. (B.5) and (B.6), PD constant, $d_{b}$ can be defined as

$$
d_{b}=\frac{2}{\pi h \delta^{2}}
$$

B.2. PD material constants, $a_{b}$ and $b_{b}$

By using Eq. (B.3) and (B.6), the strain energy per unit area for bending deformations in Eq. (25) can be rewritten in an integral form as

$$
\bar{W}_{b e n d i n g(k)}^{P D}=a_{b}\left(-\theta_{y^{\prime}, x^{\prime}(k)}+\theta_{x^{\prime}, y^{\prime}(k)}\right)^{2}+b_{b} h \int_{0}^{\delta} \int_{0}^{2 \pi}\left(\begin{array}{c}
-\theta_{y^{\prime}, x^{\prime}(k)} \cos ^{2} \varphi-\theta_{y^{\prime}, y^{\prime}(k)} \sin \varphi \cos \varphi \\
+\theta_{x^{\prime}, x^{\prime}(k)} \sin \varphi \cos \varphi+\theta_{x^{\prime}, y^{\prime}(k)} \sin ^{2} \varphi
\end{array}\right)^{2} \xi^{2} d \xi d \varphi
$$

After performing integrations given in Eq. (B.8), the strain energy per unit area for bending deformations can be written as 


$$
\begin{aligned}
\bar{W}_{\text {bending }(k)}^{P D}= & a_{b}\left(\theta_{x^{\prime}, y^{\prime}(k)}-\theta_{y^{\prime}, x^{\prime}(k)}\right)^{2} \\
& +b_{b} \frac{\pi h \delta^{3}}{12}\left[3\left(\theta_{x^{\prime}, y^{\prime}(k)}-\theta_{y^{\prime}, x^{\prime}(k)}\right)^{2}+4 \theta_{y^{\prime}, x^{\prime}(k)} \theta_{x^{\prime}, y^{\prime}(k)}+\left(\theta_{x^{\prime}, x^{\prime}(k)}-\theta_{y^{\prime}, y^{\prime}(k)}\right)^{2}\right]
\end{aligned}
$$

By comparing Eq. (B.9) and (14c), PD constants for bending deformations can be defined as

$$
b_{b}=\frac{E h^{2}}{4 \pi \delta^{3}(1+v)}, a_{b}=\frac{E h^{3}(3 v-1)}{48\left(1-v^{2}\right)}
$$

\section{Appendix C. Peridynamic constants for shear deformations}

By assuming $\bar{\theta}_{(k)} \approx \bar{\theta}_{(j)}$ for small deformations, Eq. (28) becomes

$$
\bar{W}_{\text {shear }(k)}^{P D}=\frac{1}{4} \sum_{j=1}^{N} C_{s}\left(\frac{W_{(j)}^{\prime}-W_{(k)}^{\prime}}{\xi}-\bar{\theta}_{(k)}\right)^{2} \xi V_{(j)}
$$

By using the relations in Eq. (A.2c) and (30a), the strain energy per unit area for shear deformations given in Eq. (C.1) can be rewritten in an integral form as

$\bar{W}_{\text {shear }(k)}^{P D}=\frac{1}{4} C_{s} h \int_{0}^{\delta} \int_{0}^{2 \pi}\left(\left(w_{, x^{\prime}(k)}^{\prime}+\theta_{y^{\prime}(k)}\right) \cos \varphi+\left(w_{, y^{\prime}(k)}^{\prime}-\theta_{x^{\prime}(k)}\right) \sin \varphi\right)^{2} \xi^{2} d \xi d \varphi$

By performing integrations given in Eq. (C.2), the strain energy per unit area for shear deformations can be written as

$$
\bar{W}_{\text {shear }(k)}^{P D}=\frac{1}{12} C_{s} \pi h \delta^{3}\left[\left(w_{, x^{\prime}(k)}^{\prime}+\theta_{y^{\prime}(k)}\right)^{2}+\left(w_{, y^{\prime}(k)}^{\prime}-\theta_{x^{\prime}(k)}\right)^{2}\right]
$$

By comparing Eq. (C.3) and (14d), the PD constants for shear deformations can be defined as

$$
C_{s}=\frac{3 k_{s}}{\pi \delta^{3}} \frac{E}{(1+v)}
$$

\section{Appendix D. Peridynamic constants for torsional deformations}

By using strain energy per unit area given in Eq. (15b), the potential energy corresponding to drilling rotation, $\theta_{z^{\prime}}$ can be defined as

$$
U_{\text {torsional }}=\int_{A} W_{\text {torsional }} d A=\int_{A} k_{T} \frac{E h}{2(1+v)}\left[\theta_{z^{\prime}}-\frac{1}{2}\left(v_{, x^{\prime}}^{\prime}-u_{, y^{\prime}}^{\prime}\right)\right]^{2} d A
$$

The kinetic energy corresponding to drilling rotation can be defined as

$$
T_{\theta_{z}}=\frac{1}{2} \rho h I_{z^{\prime} z^{\prime}} \dot{\theta}_{z^{\prime}}{ }^{2}
$$

By substituting Eq. (D.1) and (D.2) into Eq. (18), the Lagrangian corresponding to drilling rotation can be obtained as

$$
L_{\theta_{z}}=T_{\theta_{z}}-U_{\theta_{z}}=\frac{1}{2} \rho h I_{z^{\prime} z^{\prime}} \dot{\theta}_{z^{\prime}}{ }^{2}-\int_{A} k_{T} \frac{E h}{2(1+v)}\left[\theta_{z^{\prime}}-\frac{1}{2}\left(v_{, x^{\prime}}^{\prime}-u_{, y^{\prime}}^{\prime}\right)\right]^{2} d A
$$

As mentioned in section 3.3, since the torsional strain energy is a fictitious energy, the contribution of drilling rotation into the equation of motion other degrees of freedom can be neglected [7]. 
Therefore, the equation of motion for drilling rotation in classical continuum mechanics can be derived by using the Euler-Lagrange equation given in Eq. (17) as

$$
\frac{\rho h I_{z^{\prime} z^{\prime}}}{A} \ddot{\theta}_{z^{\prime}}=k_{T} \frac{E h}{1+v}\left[\theta_{z^{\prime}}-\frac{1}{2}\left(v_{, x^{\prime}}-u_{, y^{\prime}}\right)\right]
$$

Meanwhile, by using relations given in Eq. (A.2a) and (A.2b), the PD equation of motion for drilling rotation given in Eq. (36f) can be written in an integral form as

$$
\frac{\rho h I_{z^{\prime} z^{\prime}(k)}}{A_{(k)}} \ddot{\theta}_{z^{\prime}(k)}=C_{t} h \int_{0}^{\delta} \int_{0}^{2 \pi}\left[\frac{\theta_{z^{\prime}(k)}+\theta_{z^{\prime}(j)}}{2}-\left(\begin{array}{l}
v_{, x^{\prime}(k)}^{\prime} \cos ^{2} \varphi+v_{, y^{\prime}(k)}^{\prime} \sin \varphi \cos \varphi \\
-u_{, x^{\prime}(k)}^{\prime} \sin \varphi \cos \varphi-u_{, y^{\prime}(k)}^{\prime} \sin ^{2} \varphi
\end{array}\right)\right] \xi^{2} d \xi d \varphi
$$

By assuming $\theta_{z^{\prime}(j)} \approx \theta_{z^{\prime}(k)}$ for small deformations, Eq. (D.5) can be rewritten as

$$
\frac{\rho h I_{z^{\prime} z^{\prime}(k)}}{A_{(k)}} \ddot{\theta}_{z^{\prime}(k)}=C_{t} h \int_{0}^{\delta} \int_{0}^{2 \pi}\left[\theta_{z^{\prime}(k)}-\left(\begin{array}{l}
v_{, x^{\prime}(k)}^{\prime} \cos ^{2} \varphi+v_{, y^{\prime}(k)}^{\prime} \sin \varphi \cos \varphi \\
-u_{, x^{\prime}(k)}^{\prime} \sin \varphi \cos \varphi-u_{, y^{\prime}(k)}^{\prime} \sin ^{2} \varphi
\end{array}\right)\right] \xi^{2} d \xi d \varphi
$$

By performing integrations given in Eq. (D.6), the equation of motion for drilling rotation can be written as

$$
\frac{\rho h I_{z^{\prime} z^{\prime}(k)}}{A_{(k)}} \ddot{\theta}_{z^{\prime}(k)}=C_{t} \frac{2 \pi h \delta^{3}}{3}\left[\theta_{z^{\prime}(k)}-\frac{1}{2}\left(v_{, x^{\prime}(k)}^{\prime}-u_{, y^{\prime}(k)}^{\prime}\right)\right]
$$

By comparing Eq. (D.4) and (D.7), the PD constants for drilling rotation can be defined as

$$
C_{t}=k_{T} \frac{3}{2 \pi \delta^{3}} \frac{E}{1+v}
$$

\section{Appendix E. Adaptive Dynamic Relaxation for static and quasi-static solution}

According to Kilic and Madenci [50], the adaptive dynamic relaxation (ADR) can be used in PD solutions for static and quasi-static problems. The mass vector $\overline{\mathbf{m}}_{(k)}$ can be replaced by a mass stable vector $\mathbf{M}_{(k)}$, and damping force is added into the PD equations of motion given in Eq. (44a) for flat shells and Eq. (45a) for curved shells as

$$
\mathbf{M}_{(k)} \ddot{\mathbf{u}}_{(k)}+\mathbf{C}_{(k)} \dot{\mathbf{u}}_{(k)}=\sum_{j=1} \mu_{(k)(j)} \mathbf{f}_{(k)(j)} V_{(j)}+\mathbf{b}_{(k)}
$$

where $\mathbf{C}_{(k)}$ represents the damping matrix.

According to Underwood [51], the mass stable vector and the damping matrix can be chosen arbitrarily to get a converged solution. However, the matrix $\mathbf{M}_{(k)}$ is required to be a diagonal matrix. In this study, the mass stable vectors in global coordinates can be defined as

$$
\mathbf{M}_{(k)}=\left[\begin{array}{cccccc}
M_{u_{(k)}} & 0 & 0 & 0 & 0 & 0 \\
0 & M_{v_{(k)}} & 0 & 0 & 0 & 0 \\
0 & 0 & M_{w_{(k)}} & 0 & 0 & 0 \\
0 & 0 & 0 & M_{\theta_{x(k)}} & 0 & 0 \\
0 & 0 & 0 & 0 & M_{\theta_{y(k)}} & 0 \\
0 & 0 & 0 & 0 & 0 & M_{\theta_{z(k)}}
\end{array}\right]
$$


with

$M_{u_{(k)}}=M_{v_{(k)}}=M_{w_{(k)}}=\max \left(M_{u_{(k)}^{\prime}}^{\prime}, M_{v_{(k)}^{\prime}}^{\prime}, M_{w_{(k)}^{\prime}}^{\prime}\right)$

$M_{\theta_{x(k)}}=M_{\theta_{y(k)}}=M_{\theta_{z(k)}}=\max \left(M_{\theta_{x^{\prime}(k)}}^{\prime}, M_{\theta_{y^{\prime}(k)}^{\prime}}^{\prime}, M_{\theta_{z^{\prime}(k)}^{\prime}}^{\prime}\right)$

where $M_{u_{(k)}^{\prime}}^{\prime}, M_{v_{(k)}^{\prime}}^{\prime}, M_{w_{(k)}^{\prime}}^{\prime}, M_{\theta_{x^{\prime}(k)}}^{\prime}, M_{\theta_{y^{\prime}(k)}}^{\prime}, M_{\theta_{z^{\prime}(k)}}^{\prime}$ represent the components mass stable vector corresponding to translational and rotational DOFs in the local coordinates, $M_{u_{(k)}}, M_{v_{(k)}}, M_{w_{(k)}}$, $M_{\theta_{x(k)}}, M_{\theta_{y(k)}}, M_{\theta_{z(k)}}$ represent the components of a mass stable vector in the global coordinates. The components of the mass stable vector in the local coordinates can be calculated based on PD bond constants as

$$
\begin{aligned}
& M_{u_{(k)}^{\prime}}^{\prime}=M_{v_{(k)}^{\prime}}^{\prime} \geq \frac{1}{4} d t^{2} \frac{4 b_{i p} \pi h \delta^{2}}{\Delta x^{\prime}} \\
& M_{w_{(k)}^{\prime}}^{\prime} \geq \frac{1}{4} d t^{2} \frac{C_{s} \pi h \delta^{2}}{\Delta x^{\prime}} \\
& M_{\theta_{x^{\prime}(k)}^{\prime}}^{\prime}=M_{\theta_{y^{\prime}(k)}^{\prime}}^{\prime} \geq \frac{1}{4} d t^{2} \frac{4 b_{b} \pi h \delta^{2}}{\Delta x^{\prime}} \\
& M_{\theta_{z^{\prime}(k)}^{\prime}}^{\prime} \geq \frac{1}{4} d t^{2} \frac{C_{t} \pi h \delta^{2}}{\Delta x^{\prime}} \frac{1}{\left(\Delta x^{\prime}\right)^{2}}
\end{aligned}
$$

where $d t=1$ represents the time step for quasi-static solution [51], $\Delta x^{\prime}$ represents mesh size in the local coordinates, $b_{i p}$ represents PD constant for in-plane deformation which is given in Eq. (21d), $C_{s}$ represents PD constant for shear deformation given in Eq. (29), $b_{b}$ represents PD constant for bending deformation given in Eq. (26b), $C_{t}$ represents PD constant for torsional deformation given in Eq. (32). 


\section{Appendix F. Peridynamic prediction results for thin shells}

In order to verify the capability of the developed PD model for thin shell, the same problems discussed in this Section 7.1 and 7.2 are investigated with thinner shells. In this section, the PD prediction results for both flat shell in Section 7.1 and curved shell in Section 7.2 with $L / h=100$ are presented.

\section{F.1. Flat shell with $L / h=100$ subjected to loads $p_{x}=1 \times 10^{2} \mathrm{~N} / \mathrm{m}, p_{z}=-1 \times 10^{2} \mathrm{~N} / \mathrm{m}$}

Figs. F1-F8 show the comparison between PD prediction results with FEA results for thin flat shell with $L / h=100$. The mesh size $150 \times 150$ is used in both PD and FEA solutions. As can be seen from the figures, the PD and FEA solution results much very well which show the accuracy of the developed PD model for thin shell.

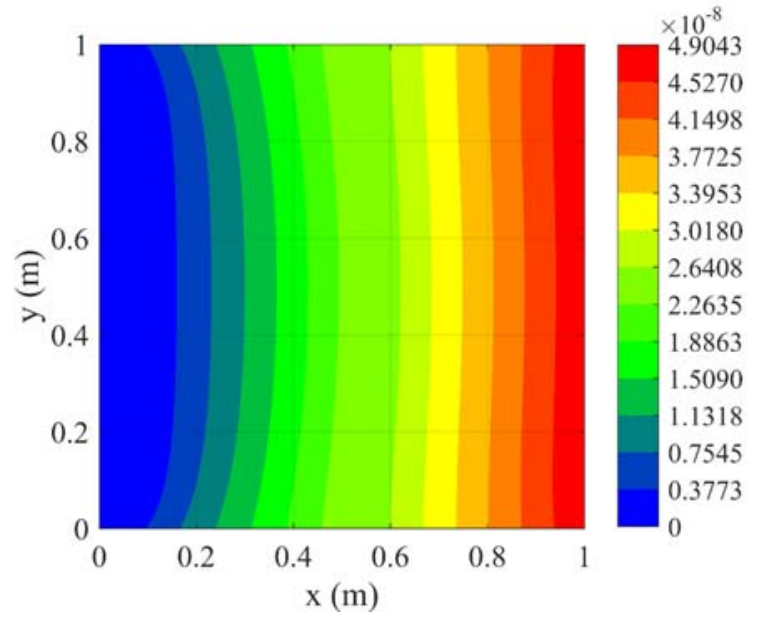

(a)

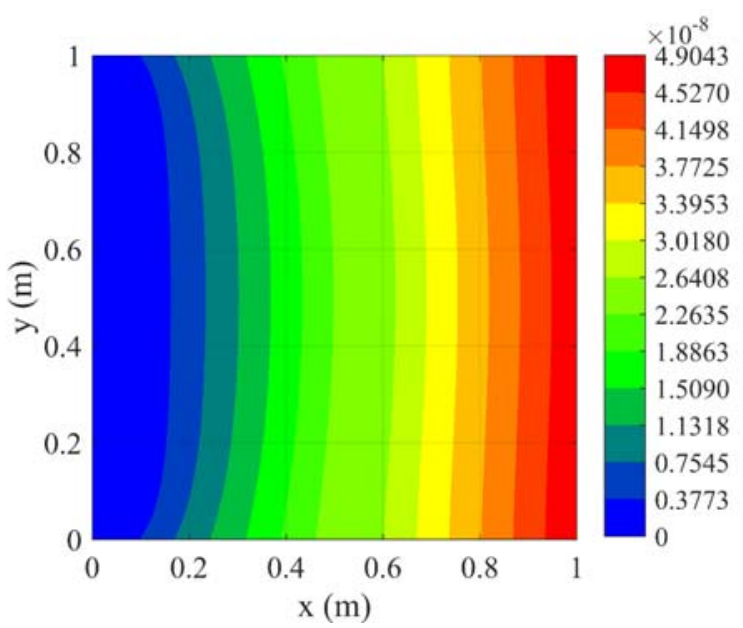

(b)

Fig. F1. Variation of displacement $u(\mathrm{~m})$ of shell with $L / h=100$ (a) PD, (b) FEA results

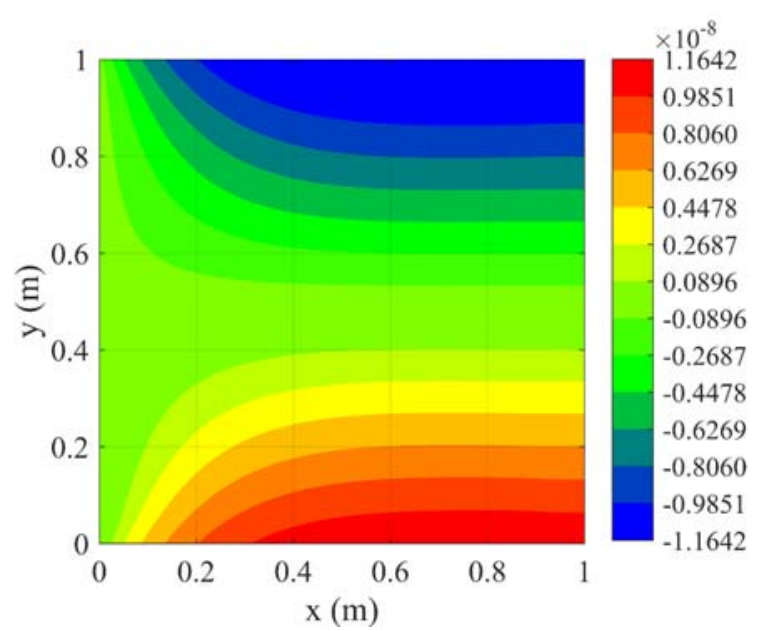

(a)

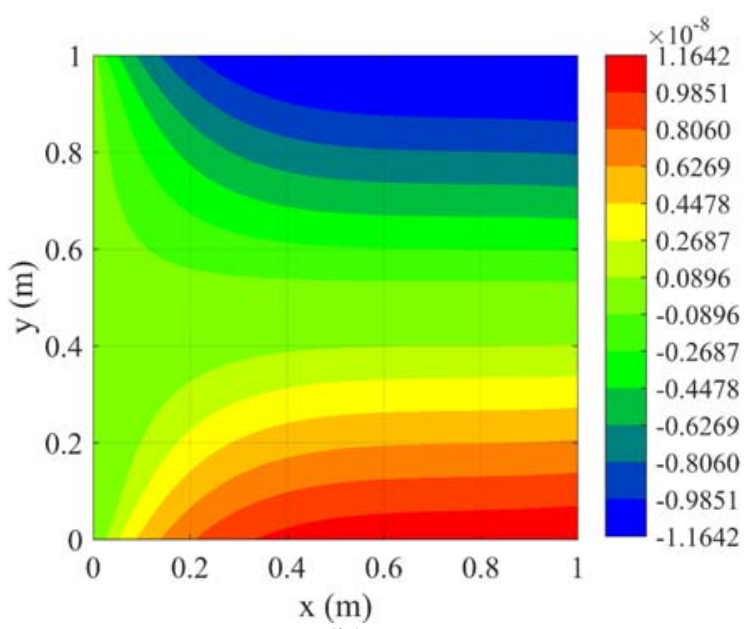

(b)

Fig. F2. Variation of displacement $v(\mathrm{~m})$ of shell with $L / h=100$ (a) PD, (b) FEA results 


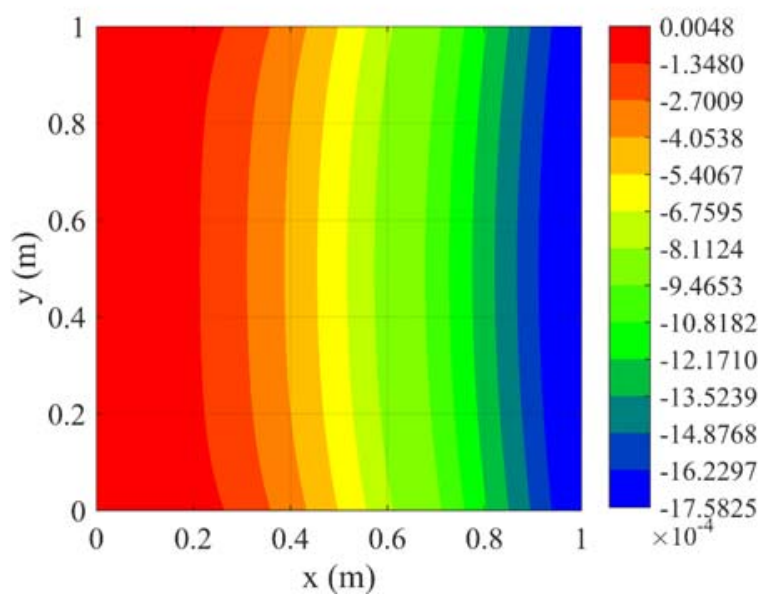

(a)

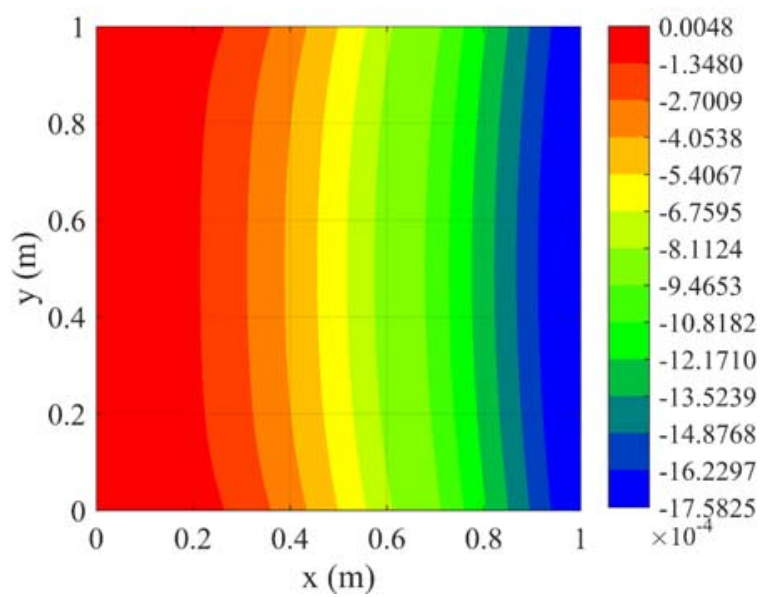

(b)

Fig. F3. Variation of displacement $w(\mathrm{~m})$ of shell with $L / h=100$ (a) PD, (b) FEA results

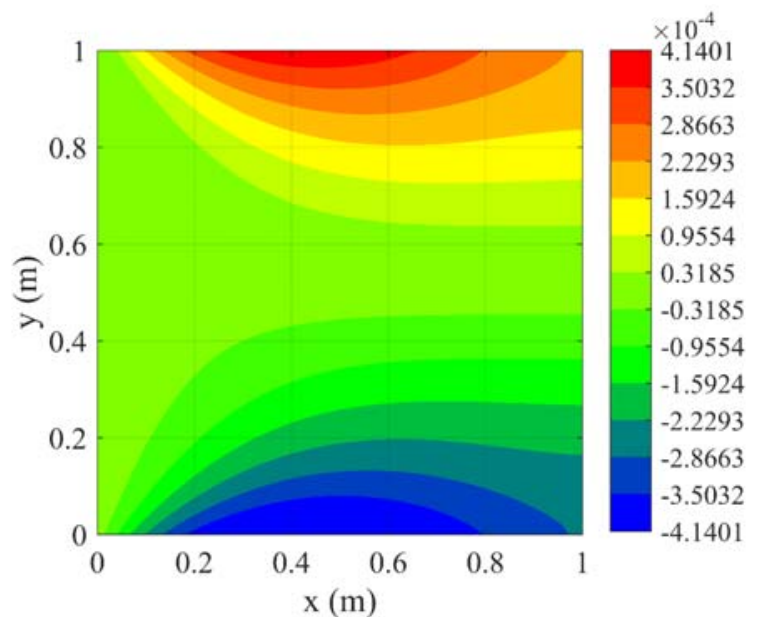

(a)

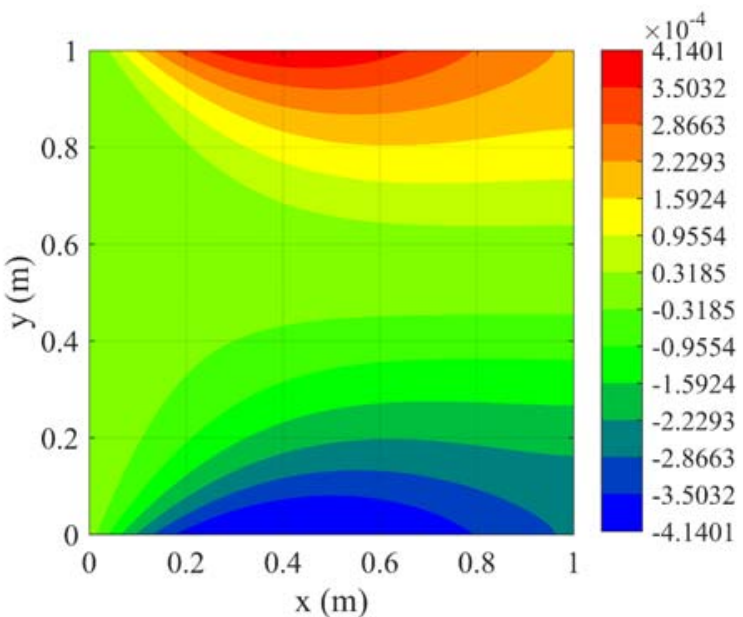

(b)

Fig. F4. Variation of rotation $\theta_{x}(\mathrm{rad})$ of shell with $L / h=100$ (a) PD, (b) FEA results 


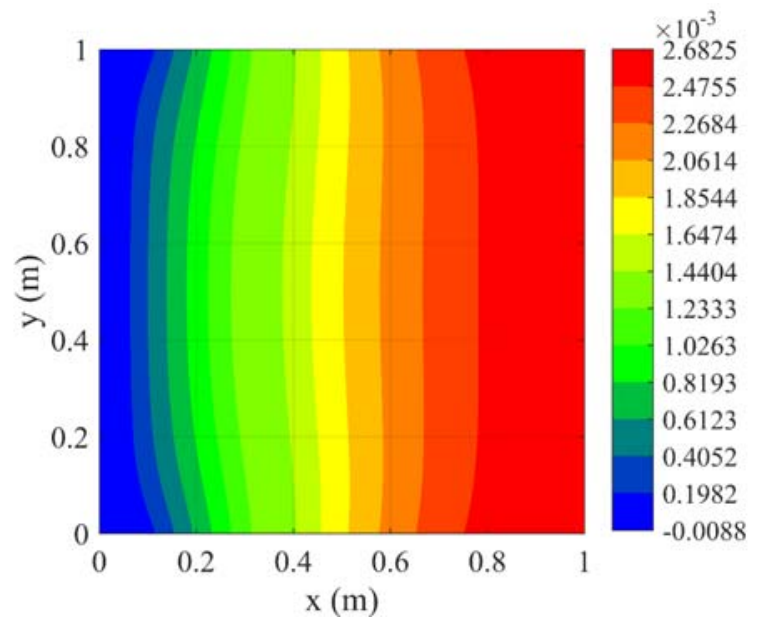

(a)

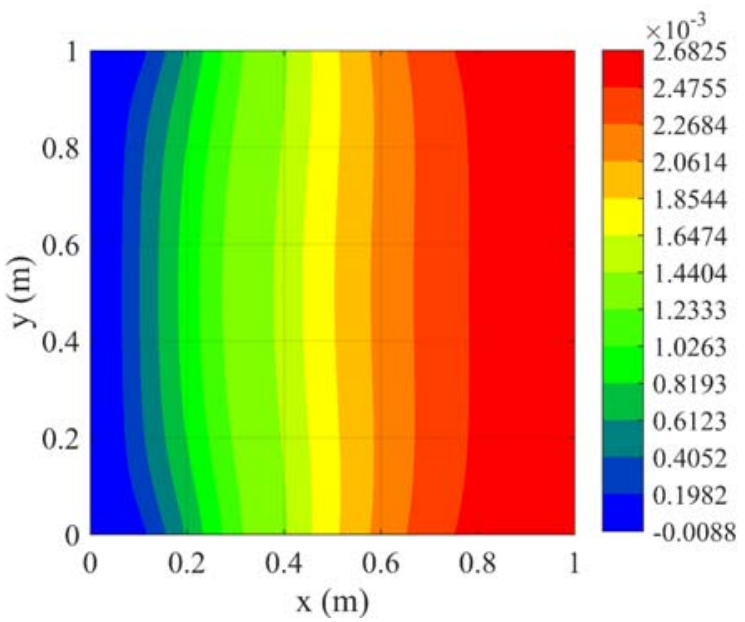

(b)

Fig. F5. Variation of rotation $\theta_{y}(\mathrm{rad})$ of shell with $L / h=100$ (a) PD, (b) FEA results

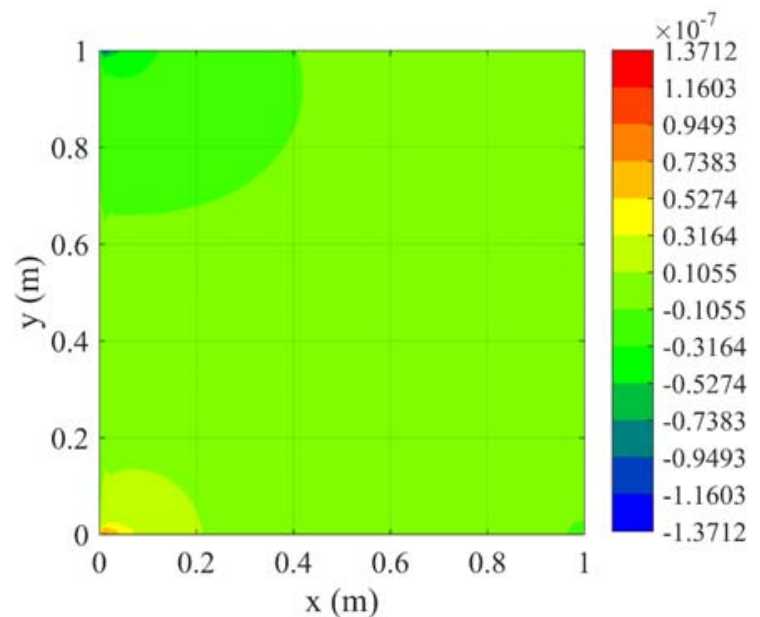

(a)

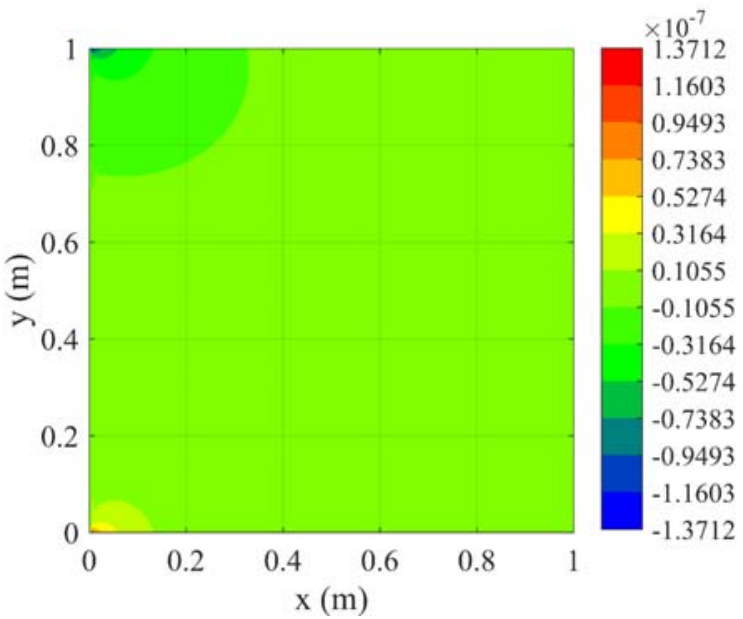

(b)

Fig. F6. Variation of rotation $\theta_{z}(\mathrm{rad})$ of shell with $L / h=100$ (a) PD, (b) FEA results

Fig. F7 and Fig. F8 show the comparison between the PD and FEA solution results along $y=W / 2$ and $x=L / 2$, respectively. As can be seen from the figures, PD and FEA solution results agree very well for both in-plane and out-of-plane deformations. Therefore, the developed PD model for thin flat shells is verified. 

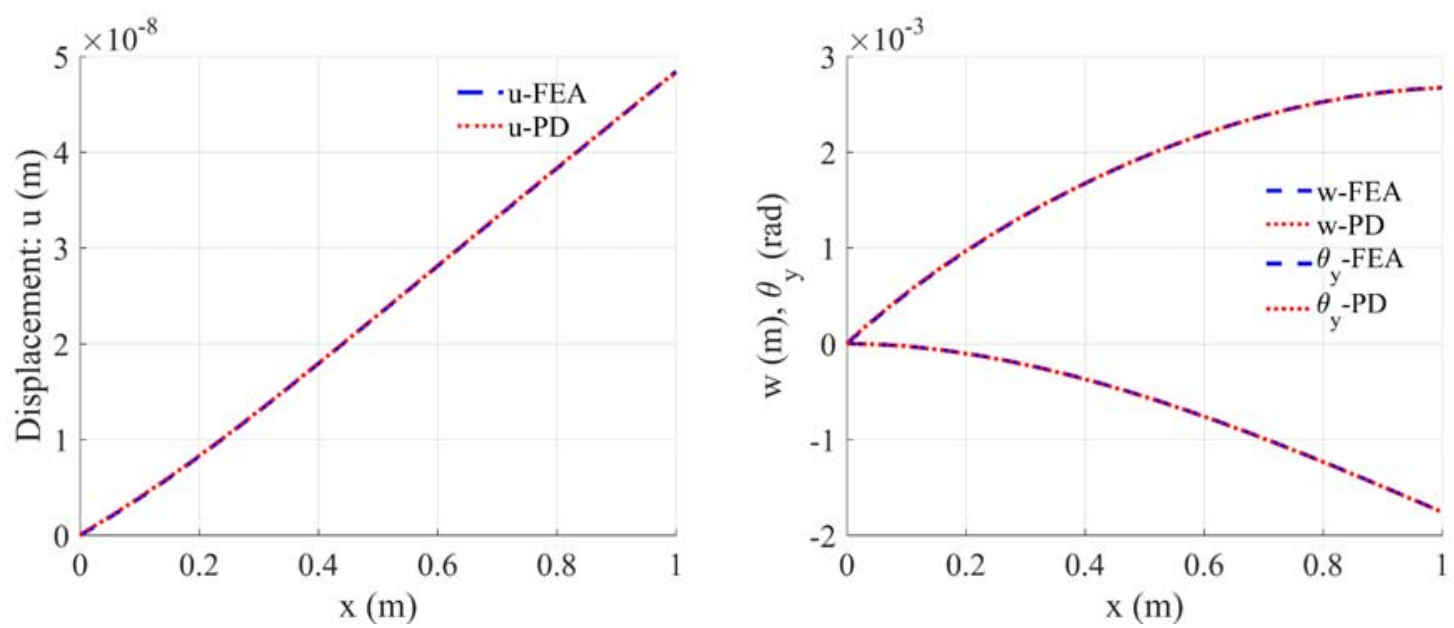

Fig. F7. Variation of DOFs $u(\mathrm{~m}), w(\mathrm{~m}), \theta_{y}(\mathrm{rad})$ along $y=W / 2$
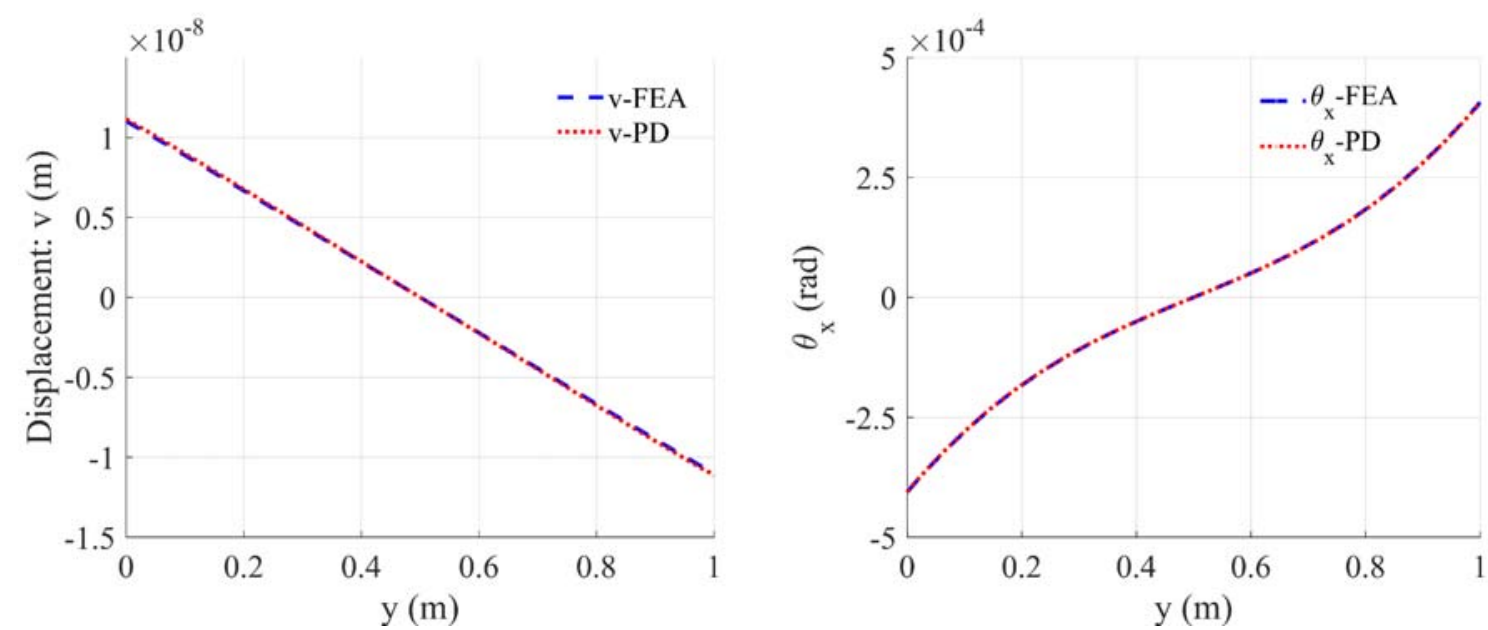

Fig. F8. Variation of DOFs $v(\mathrm{~m}), \theta_{x}(\mathrm{rad})$ along $x=L / 2$

F.2. curved shell with $L / h=100$ subjected to loads $p_{z}=-10 \mathrm{~N} / \mathrm{m}$

Figs. F9-F15 show the comparison between PD prediction results with FEA results for curved shell $R=3 \mathrm{~m}$ with $L / h=100$. The mesh size $150 \times 150$ is used in both PD and FEA solutions. As can be seen from the figures, the PD and FEA solution results much very well which show the accuracy of the developed PD model for thin shell.

Fig. F15 shows the deformed shape along $x=L / 2$ of the curved shell with the displacements are magnified 100 times. It can be seen that PD and FEA solution results have very good agreement. Therefore, it can be concluded that the capability of the developed PD model for thin shell is validated. 


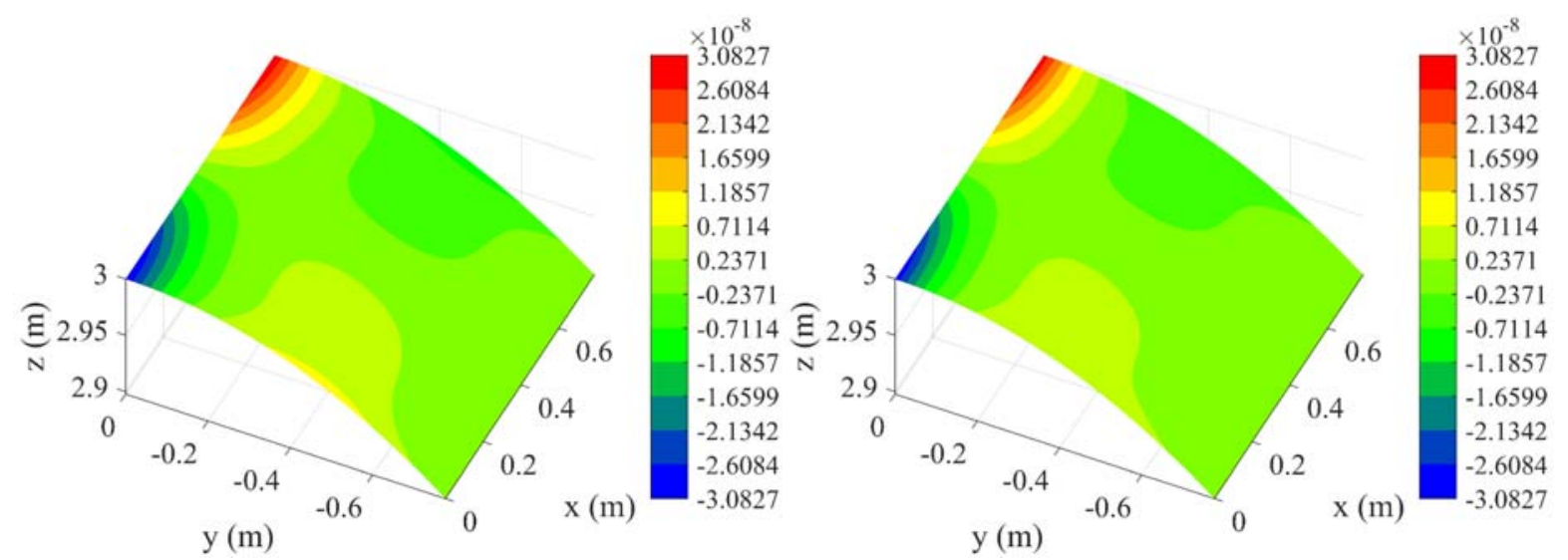

Fig. F9. Variation of displacement $u(\mathrm{~m})$ of curved shell $R=3 \mathrm{~m}$ with $L / h=100$ (a) PD, (b) FEA results

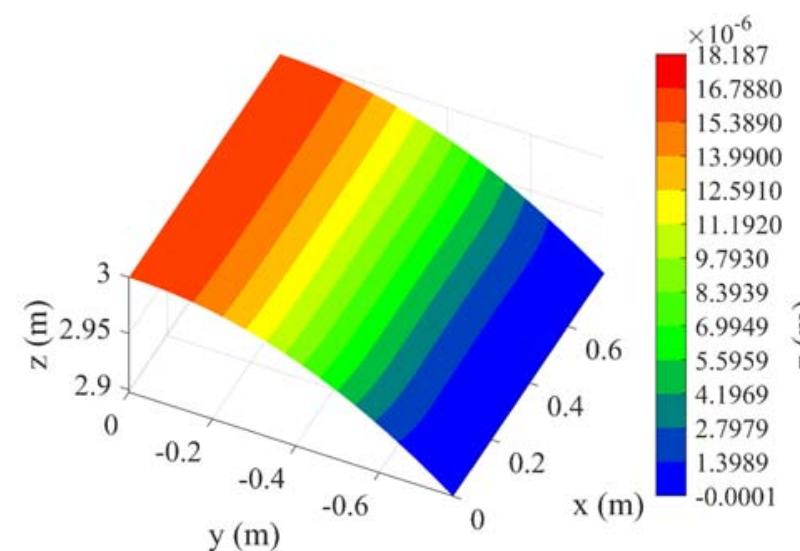

(a)

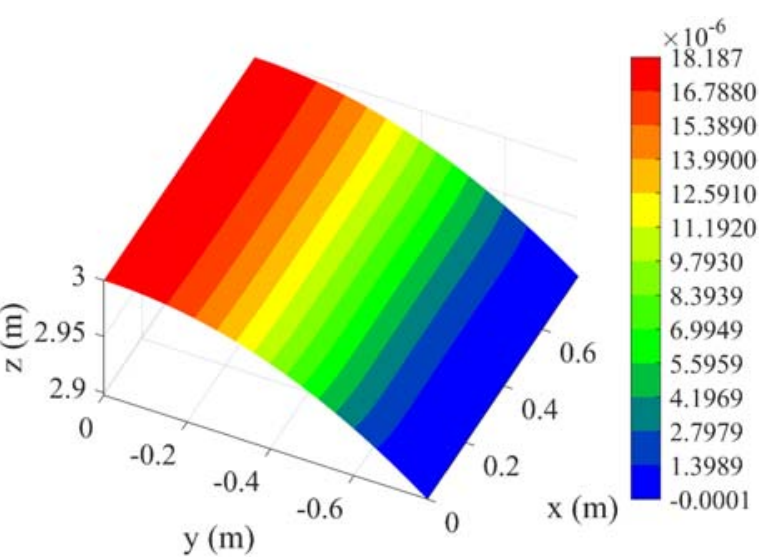

(b)

Fig. F10. Variation of displacement $v(\mathrm{~m})$ of curved shell $R=3 \mathrm{~m}$ with $L / h=100$ (a) PD, (b) FEA results

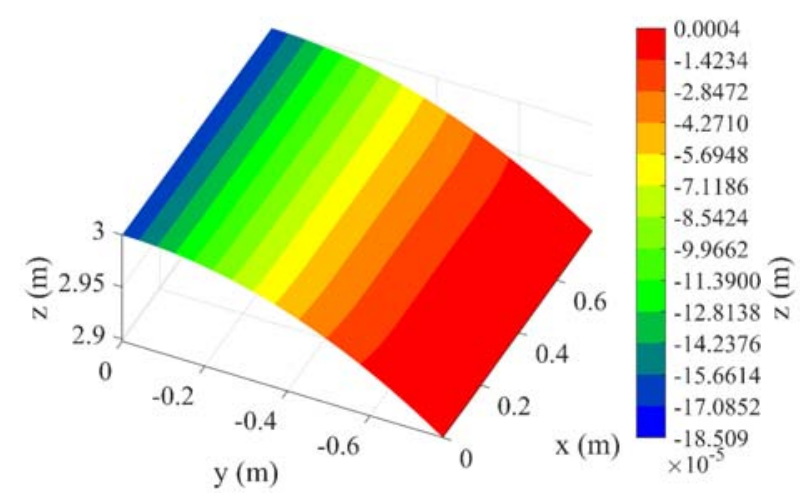

(a)

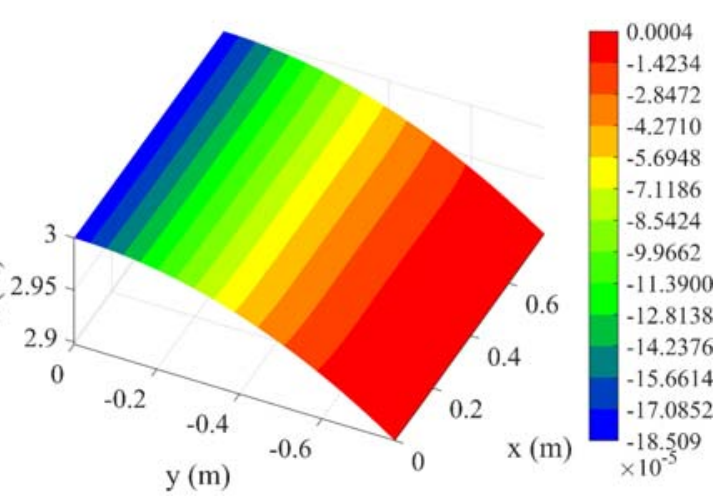

(b)

Fig. F11. Variation of displacement $w(\mathrm{~m})$ of curved shell $R=3 \mathrm{~m}$ with $L / h=100$ (a) PD, (b) FEA results 


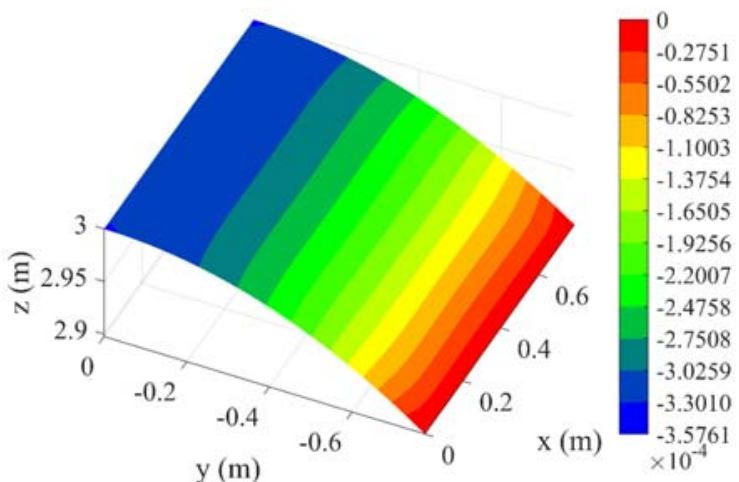

(a)

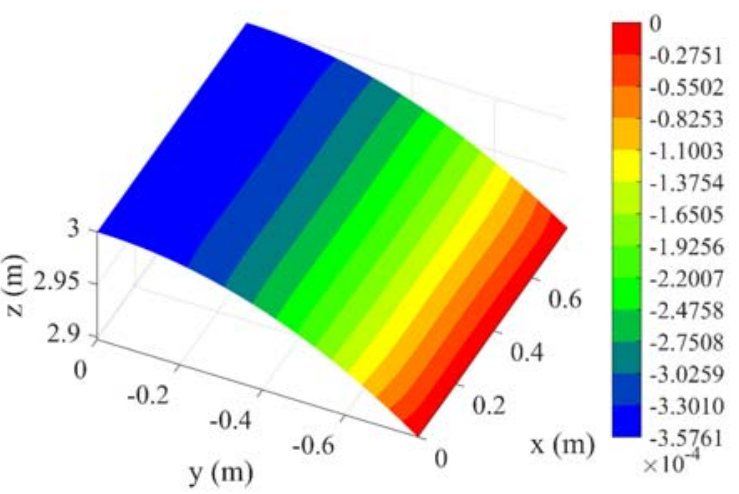

(b)

Fig. F12. Variation of rotation $\theta_{x}(\mathrm{rad})$ of curved shell $R=3 \mathrm{~m}$ with $L / h=100$ (a) PD, (b) FEA results

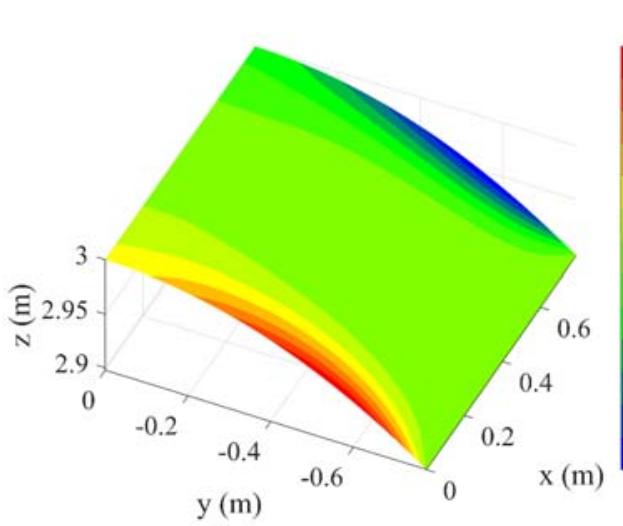

(a)
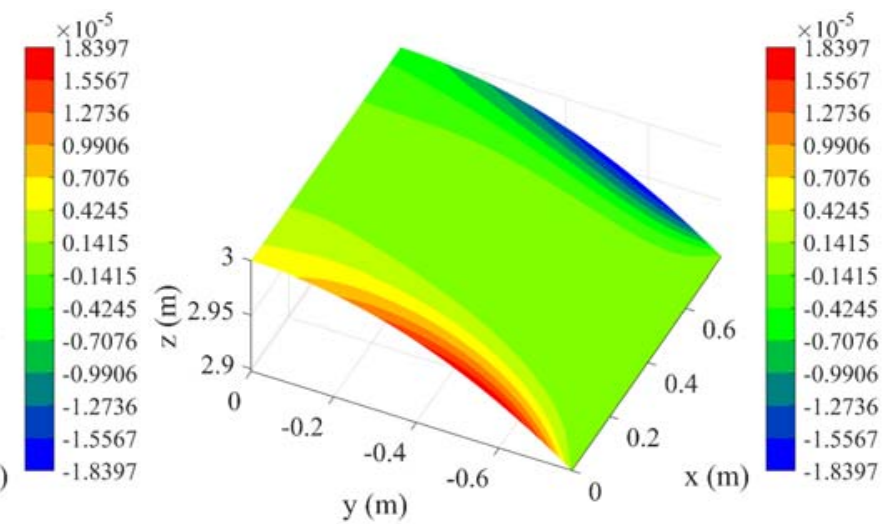

(b)

Fig. F13. Variation of rotation $\theta_{y}(\mathrm{rad})$ of curved shell $R=3 \mathrm{~m}$ with $L / h=100$ (a) PD, (b) FEA results

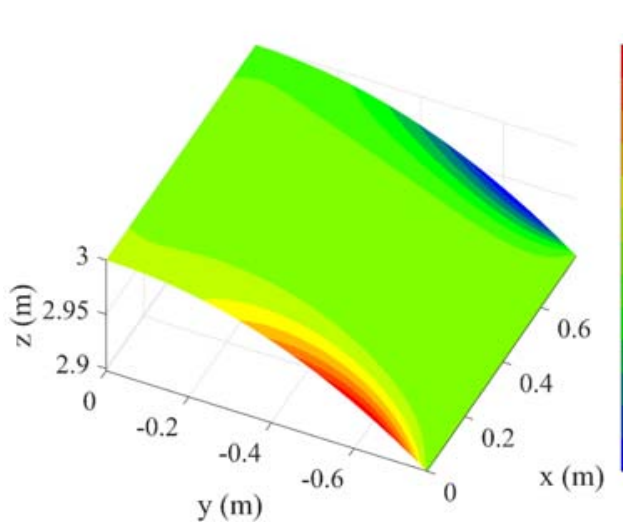

(a)

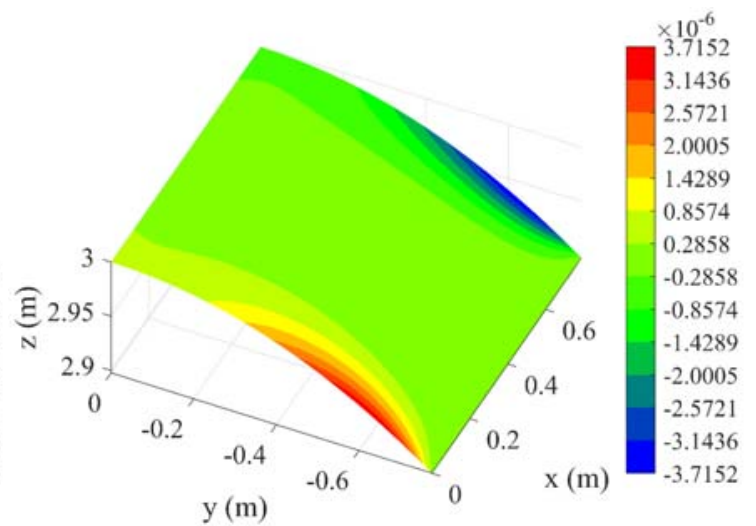

(b)

Fig. F14. Variation of rotation $\theta_{z}(\mathrm{rad})$ of curved shell $R=3 \mathrm{~m}$ with $L / h=100$ (a) PD, (b) FEA results 


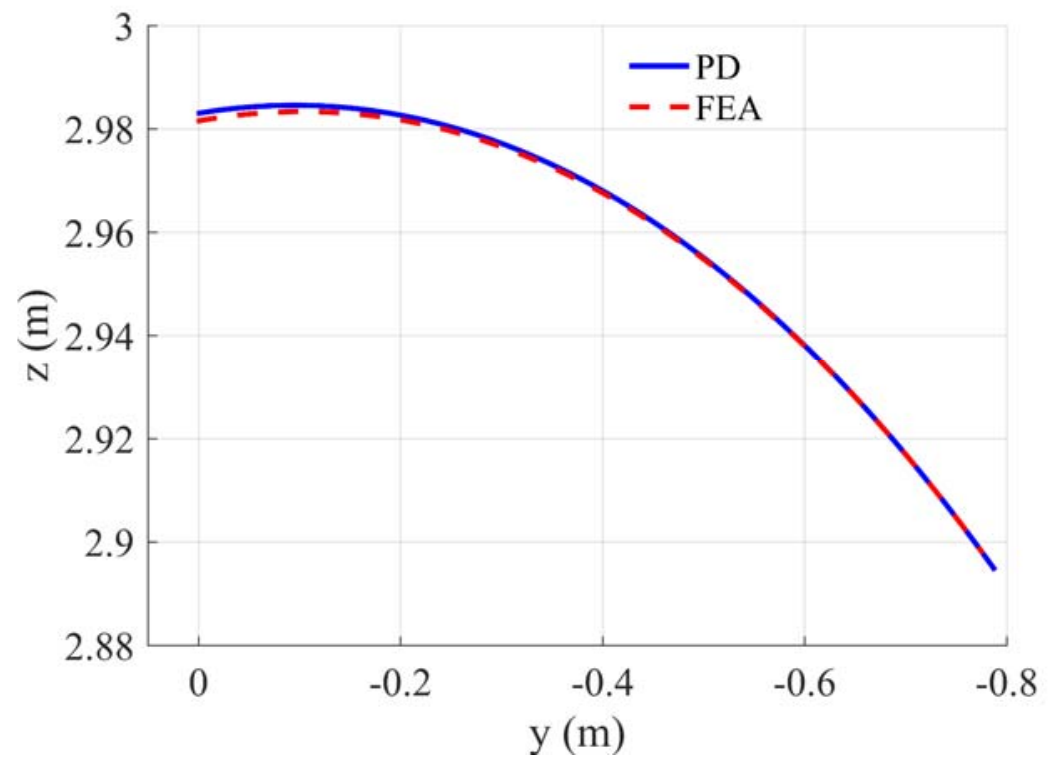

Fig. F15. Deformed configuration along $x=L / 2$ (displacements are magnified by 100 times) 


\section{References}

[1] Silling SA. Reformulation of elasticity theory for discontinuities and long-range forces. Journal of the Mechanics and Physics of Solids. 2000 48(1):175-209.

[2] Silling SA, Askari E. A meshfree method based on the peridynamic model of solid mechanics. Computers \& structures. 2005;83(17-18):1526-35.

[3] Mindlin RD. Influence of rotatory inertia and shear on flexural motions of isotropic, elastic plates. J. appl. Mech. 1951;18:31-8.

[4] Reissner E. The effect of transverse shear deformation on the bending of elastic plates. J. appl. Mech. 1945:A69-77.

[5] Reissner E. On the theory of transverse bending of elastic plates. International Journal of Solids and Structures. 1976;12(8):545-54.

[6] Wisniewski K. Finite rotation shells. Basic equations and finite elements for Reissner kinematics. CIMNE-Springer. 2010.

[7] Kanok-nukulchai W. A simple and efficient finite element for general shell analysis. International Journal for Numerical Methods in Engineering. 1979;14(2):179-200.

[8] Dvorkinq EN. A formulation of general shell of tensorial componentst. international journal for numerical methods in engineering. 1986;22:722.

[9] Cook RD. Four-node 'flat'shell element: drilling degrees of freedom, membrane-bending coupling, warped geometry, and behavior. Computers \& structures. 1994;50(4):549-55.

[10] Bathe KJ. Finite element procedures. Klaus-Jurgen Bathe; 2006.

[11] Chapelle D, Bathe KJ. The finite element analysis of shells-Fundamentals. Springer Science \& Business Media; 2010.

[12] Sussman T, Bathe KJ. 3D-shell elements for structures in large strains. Computers \& Structures. 2013;122:2-12.

[13] Silling SA, Bobaru F. Peridynamic modeling of membranes and fibers. International Journal of Non-Linear Mechanics. 2005;40(2-3):395-409.

[14] Madenci E, Oterkus E. Peridynamic theory and its applications. New York: Springer; 2014.

[15] Breitenfeld, M.S., Geubelle, P.H., Weckner, O. and Silling, S.A., 2014. Non-ordinary statebased peridynamic analysis of stationary crack problems. Computer Methods in Applied Mechanics and Engineering, 272, pp.233-250.

[16] Sarego, G., Le, Q.V., Bobaru, F., Zaccariotto, M. and Galvanetto, U., 2016. Linearized statebased peridynamics for 2-D problems. International Journal for Numerical Methods in Engineering, 108(10), pp.1174-1197.

[17] O'Grady J, Foster J. Peridynamic plates and flat shells: A non-ordinary, state-based model. International Journal of Solids and Structures. 2014;51(25-26):4572-9.

[18] Diyaroglu C, Oterkus E, Oterkus S, Madenci E. Peridynamics for bending of beams and plates with transverse shear deformation. International Journal of Solids and Structures. 2015;69:152-68. [19] Nguyen CT, Oterkus S. Peridynamics formulation for beam structures to predict damage in offshore structures. Ocean Engineering. 2019;173:244-67.

[20] Yang Z, Oterkus E, Nguyen CT, Oterkus S. Implementation of peridynamic beam and plate formulations in finite element framework. Continuum Mechanics and Thermodynamics. 2018:15 .

[21] Chowdhury, S.R., Roy, P., Roy, D. and Reddy, J.N., 2016. A peridynamic theory for linear elastic shells. International Journal of Solids and Structures, 84, pp.110-132.

[22] Kilic B, Madenci E. Peridynamic theory for thermomechanical analysis. IEEE Transactions on Advanced Packaging. 2010;33(1):97-105. 
[23] Alpay S, Madenci E. Crack growth prediction in fully-coupled thermal and deformation fields using peridynamic theory. In54th AIAA/ASME/ASCE/AHS/ASC structures, structural dynamics, and materials conference 2013 (p. 1477).

[24] Oterkus S, Madenci E, Agwai A. Fully coupled peridynamic thermomechanics. Journal of the Mechanics and Physics of Solids. 2014;64:1-23.

[25] Oterkus S, Madenci E. Peridynamics for Fully Coupled Thermomechanical Analysis of Fiber Reinforced Laminates. In55th AIAA/ASMe/ASCE/AHS/SC Structures, Structural Dynamics, and Materials Conference 2014 (p. 0694).

[26] Amani J, Oterkus E, Areias P, Zi G, Nguyen-Thoi T, Rabczuk T. A non-ordinary state-based peridynamics formulation for thermoplastic fracture. International Journal of Impact Engineering. 2016;87:83-94.

[27] Madenci E, Oterkus S. Ordinary state-based peridynamics for plastic deformation according to von Mises yield criteria with isotropic hardening. Journal of the Mechanics and Physics of Solids. 2016;86:192-219.

[28] Madenci E, Oterkus S. Ordinary state-based peridynamics for thermoviscoelastic deformation. Engineering Fracture Mechanics. 2017;175:31-45.

[29] Gao Y, Oterkus S. Ordinary state-based peridynamic modelling for fully coupled thermoelastic problems. Continuum Mechanics and Thermodynamics. 2018;9:1-31.

[30] Gao Y, Oterkus S. Fully coupled thermomechanical analysis of laminated composites by using ordinary state based peridynamic theory. Composite Structures. 2019;207:397-424.

[31] Oterkus S, Madenci E. Peridynamic modeling of fuel pellet cracking. Engineering Fracture Mechanics. 2017;176:23-37.

[32] Gao Y, Oterkus S. Peridynamic analysis of marine composites under shock loads by considering thermomechanical coupling effects. Journal of Marine Science and Engineering. 2018;6(2):38.

[33] Xu, Z., Zhang, G., Chen, Z. and Bobaru, F., 2018. Elastic vortices and thermally-driven cracks in brittle materials with peridynamics. International Journal of Fracture, 209(1-2), pp.203-222.

[34] Hetnarski RB, Eslami MR, Gladwell GM. Thermal stresses: advanced theory and applications. New York: Springer; 2009.

[35] Ugural AC, Fenster SK. Advanced strength and applied elasticity. Pearson education; 2003.

[36] Diyaroglu C. Peridynamics and its applications in marine structures (Doctoral dissertation, University of Strathclyde);2016.

[37] Bhatti MA. Advanced topics in finite element analysis of structures: with Mathematica and MATLAB computations. John Wiley \& Sons, Inc.; 2006.

[38] Macek, R.W. and Silling, S.A., 2007. Peridynamics via finite element analysis. Finite Elements in Analysis and Design, 43(15), pp.1169-1178.

[39] Yuan, H., Wang, F., Liu, Y., Bian, H., Chen, W. and Wang, Y., 2018. Time-dependent behavior of subcritical crack growth for rock plate: Experimental and numerical study. International Journal of Distributed Sensor Networks, 14(11), p.1550147718812019.

[40] Atkinson, B.K., 1979, February. Fracture toughness of Tennessee sandstone and Carrara marble using the double torsion testing method. In International Journal of Rock Mechanics and Mining Sciences \& Geomechanics Abstracts (Vol. 16, No. 1, pp. 49-53). Pergamon.

[41] Kalthoff, J.F., 1988. Shadow optical analysis of dynamic shear fracture. Optical Engineering, 27(10), p.271035.

[42] Morey GW. Properties of glass. Reinhold Publishing Corporation; New York.; 1938. 
[43] Kitching R. Pressurised eggshell analysis. International journal of mechanical sciences. 1997;39(4):391-6.

[44] Macleod N, Bain MM, Hancock JW. The mechanics and mechanisms of failure of hens' eggs. International journal of fracture. 2006;142(1-2):29-41.

[45] Simeonovová J, Buchar J. On the identification of the eggshell elastic properties under quasistatic compression. Acta Universitatis Agriculturae et Silviculturae Mendelianae Brunensis. 2015;52(5):73-82.

[46] Rahn H, Paganelli CV. Shell mass, thickness and density of avian eggs derived from the tables of Schönwetter. Journal für Ornithologie. 1989;130(1):59-68.

[47] Taylor D, Walsh M, Cullen A, O'Reilly P. The fracture toughness of eggshell. Acta biomaterialia. 2016;37:21-7.

[48] Kumbár V, Strnková J, Nedomová Š, Buchar J. Fluid dynamics of liquid egg products. Journal of biological physics. 2015;41(3):303-11.

[49] Hincke, M.T., Nys, Y., Gautron, J., Mann, K., Rodriguez-Navarro, A.B. and McKee, M.D., 2012. The eggshell: structure, composition and mineralization. Front Biosci, 17(1266), p.80.

[50] Kilic B, Madenci E. An adaptive dynamic relaxation method for quasi-static simulations using the peridynamic theory. Theoretical and Applied Fracture Mechanics. 2010;53(3):194-204.

[51] Underwood P. Dynamic relaxation. Computational method for transient analysis. 1986;1:24563. 